\author{
La formule de Minkowski-Siegel \\ pour les formes bilinéaires symétriques \\ non dégénérées et définies positives
}




\section{La formule de Minkowski-Siegel pour les formes bilinéaires symétriques non dégénérées et définies positives}

Maurice Mischler

Ce travail a été effectué en vue de l'obtention du Diplôme de mathématicien de l'Université de Lausanne, sous la direction du Professeur Jacques Boéchat 


\section{Introduction}

Une étape importante de l'histoire des formes bilinéaires symétriques entières commence lorsque, vers 1859, Hermite démontre qu'il n'y a qu'un nombre fini de classes d'équivalence pour un déterminant et une dimension $n$ donnés. Quand un objet mathématique possède un nombre fini d'éléments, il est naturel de se demander quel est ce nombre.

Nous allons nous intéresser aux formes non dégénérées sur $\mathbb{Z}$ et définies positives. Hermite lui-même démontra que si $1 \leq n \leq 7$, il n'y avait qu'une classe d'équivalence.

Mordell montre, en 1938, que si $n=8$, il y a exactement deux classes.

En 1957, Kneser énumère toutes les formes jusqu'à 16 variables.

Il apparaît que si $n \equiv 0 \bmod 8$, il est possible de trouver de telles formes $\beta$ telles que $\beta(x, x)$ soit pair pour tout $x$. Ces formes sont appelée "formes de type $I I$ " ou "paires", sinon, on dit qu'elles sont de "type I", ou "impaires".

Niemeier en 1968 donna la liste de toute les formes paires à 24 variables : il y en a exactement 24.

Conway et Sloane, en 1982, ont donné toutes les formes jusqu'à $n=24$, puis avec Borcherds jusqu'à $n=25$. Une liste de ces résultats est donnée dans ce travail au chapitre 5 .

Mais alors, que vient faire la formule de Siegel dans tout cela?

Imaginez que vous possédez une certaine quantité de classes de formes bilinéaires symétriques, définies positives, dans un type donné, et pour une dimension $n$ donnée . Eh bien, la formule de Siegel permet de dire si oui ou non votre liste est complète.

Plus précisément, soit $M=M_{1}$ un $\mathbb{Z}$-module bilinéaire symétrique, défini positif et de dimension $n$. Soient $M_{2}, \ldots, M_{k}$ des représentants des classes d'équivalence dans le même type que $M$. Pour chacun de ces modules, on pose $O\left(M_{i}\right)$ le groupe orthogonal de $M_{i}$. Ce groupe est fini dans notre cas. La formule de Siegel nous donne alors pour tout $n$ et pour tout type la somme

$$
\sum_{i=1}^{k} \frac{1}{\left|O\left(M_{i}\right)\right|}
$$

Cette formule est donnée dans les cas qui nous intéressent dans [4, ch.16, thm. 1 et 2].

Or, les auteurs de cet ouvrages nous avertissent qu'un bon nombres d'articles concernant cette formule comportent des erreurs (notament [9] et [12]).

Le but de ce travail est donc de reprendre la théorie de Siegel, exposée par Kneser dans [7]. Cela est fait dans le chapitre 2, alors que le premier chapitre est consacré au rappel de certain résultats classiques concernant les formes bilinéaires.

La théorie étant élaborée, il reste les calculs à faire pour obtenir la formule explicitement pour chacun des types. Pour cela, nous devons calculer les cardinaux des groupes orthogonaux sur les corps finis, et sur $\mathbb{Z} / 8 \mathbb{Z}$. Ces calculs sont donnés dans les chapitres 3 et 4 .

Enfin, le chapitre 5 est consacré au calcul proprement dit de cette formule.

Je tiens à exprimer toute ma gratitude au professeur Jacques Boéchat qui, avec une patiente attention, m'a aidé à écrire ce diplôme, et sans qui ce travail n'aurait pas vu le jour.

Je remercie aussi le professeur Henri Joris qui a aimablement été d'accord d'être l'expert de ce travail. Enfin, je remercie Monique d'avoir bien voulu lire ce travail afin d'éliminer les principales fautes de rédaction. 


\section{Table des matières}

Introduction

Chapitre 1 : Définitions et propriétés classiques des formes bilinéaires et quadratiques. 3
A. Formes bilinéaires et formes quadratiques.
B. Anneaux et corps p-adiques.
C. Réseaux et bases de réseaux.
D. Réseaux bilinéaires et quadratiques.
E. Quelques rappels.
F. La notion de genre.
G. Enoncé du problème.

Chapitre 2 : Mesures, masses et formule de Minkowski-Siegel.

A. Structure congruentielle ct mesure de Haar. 20

B. Groupe orthogonal et structure congruentielle. 22

C. Groupe orthogonal adélique el structure congruentielle. 24

$\begin{array}{lr}\text { D. Lien entre } O(V) \text { et } \widetilde{O}(V) . & 28\end{array}$

E. Domaine fondamentale et masse. $\quad 29$

F. Représentations. $\quad 32$

G. Formule de Siegel. $\quad 36$

H. Normalisation des $\mu_{p} . \quad 40$

Chapitre 3 : Le groupe orthogonal sur les corps $\mathbb{F}_{p}$. 46
A. Formes quadratiques non dégénérées sur 2 .
B. Formes quadratiques sur $p, p$ impair.
C. Le cardinal du groupe orthogonal.

Chapitre 4 : Le groupe orthogonal modulo 8.

A. Groupes orthogonaux quadratiques et bilinéaires. 52

B. Les vecteurs de norme $i$. 53

$\begin{array}{lr}\text { C. Le cardinal du groupe } O_{\beta}^{n} . & 56\end{array}$

Chapitre 5 : Calcul explicite de la formule de Minkowski-Siegel pour los formes entières et définies positives.

A. La formule de Minkowski-Siegel dans le cas de $\mathscr{C}_{n}$.

B. La formule de Minkowski-Siegel darns le cas de $\mathscr{L}_{n}$.

C. Applications et conclusion. 


\section{CHAPITRE 1}

\section{Définitions et propriétés classiques des formes bilinéaires et quadratiques.}

Ce premier chapitre sera essentiellement consacré au rappel de certains résultats classiques relatifs aux formes bilinéaires et quadratiques ainsi qu'aux objets dont nous aurons besoin pour ce travail.

\section{A. Formes bilinéaires et formes quadratiques.}

\section{Définitions 1.1}

Soient $A$ un anneau unitaire commutatif, et $M$ un $A$-module. Une forme bilinéaire est une application

$$
\beta: M \times M \longrightarrow A \text { telle que } \begin{aligned}
\beta(x+y, z) & =\beta(x, z)+\beta(y, z) \\
\beta(x, y+z) & =\beta(x, y)+\beta(x, z) \\
\beta(\lambda x, y) & =\lambda \beta(x, y)=\beta(x, \lambda y) \quad \forall x, y, z \in M, \text { et } \lambda \in A .
\end{aligned}
$$

On dit que $\beta$ est une forme bilinéaire symétrique si $\beta(x, y)=\beta(y, x) \forall x, y \in M$.

La plupart du temps, $\beta$ sera supposée non dégénérée, c'est-à-dire qu'elle sera symétrique, et que l'homomorphisme

$$
\begin{aligned}
f_{\beta}: M & \longrightarrow \operatorname{Hom}_{A}(M, A):=M^{*} \\
x & \longmapsto \beta(x, \cdot)
\end{aligned}
$$

sera un isomorphisme.

$(M, \beta)$ est alors appelé module bilinćaire.

Deux modules bilinéaires $(M, \beta)$ et $\left(M^{\prime}, \beta^{\prime}\right)$ sont dits équivalents s'il existe un isomorphisme $u: M \longrightarrow M^{\prime}$ tel que $\beta^{\prime}(u(x), u(y))=\beta(x, y) \forall x, y \in M$, et on note $(M, \beta) \stackrel{A}{\simeq}\left(M^{\prime}, \beta^{\prime}\right)$; nous écrirons souvent par abus que $\beta \simeq \beta^{\prime}$, ou alors $M \simeq M^{\prime}$, s'il n'y a pas d'ambiguïté.

\section{Proposition 1.2}

Si $(M, \beta)$ est un $A$-module quadratique libre de rang $n$, et $\left(e_{1}, \ldots, e_{n}\right)$ est une base de $M$, on note $M_{\beta}$ la matrice à coefficient dans $A$ définie par $M_{\beta_{i j}}=\beta\left(e_{i}, e_{j}\right) \forall i, j \in \mathbb{N}_{n}$.

$\beta$ est non dégénérée si et seulement si $\operatorname{det}\left(M_{\beta}\right)$ est une unité de $A$. Nous noterons $U(A)$, l'ensemble $\operatorname{des}$ unités de $A$.

De plus, il y a équivalence entre le fait que $(M, \beta) \simeq\left(M^{\prime}, \beta^{\prime}\right)$ et l'existence d'une matrice $S$ inversible dans $M_{n}(A)$ telle que $S M_{\beta}^{\prime} S^{t}=M_{\beta}$.

\section{Démonstration :}

Le premier point découle du fait que $M^{*}$ peut être muni de la base $\left(e_{1}^{\#}, \ldots, e_{n}^{\#}\right)$

où $e_{i}^{\#}\left(e_{j}\right)=\delta_{i j} \forall i, j \in I_{n}$ et que $f_{\beta}\left(e_{i}\right)=\sum_{j=1}^{n} f_{i j} e_{j}^{\#}$. La matrice $\left(f_{i j}\right)_{i, j \in N_{n}}$ de $f_{\beta}$ n'est autre que $M_{\beta}$.

Puisque $f_{\beta}$ est un isomorphisme, on conclut.

Le second point découle aussi directement de la définition: $S$ est la transposée de la matrice de $u$.

\section{Remarque :}

Dorénavant, si cela n'est pas explicitement mentionné, $M$ sera supposé libre de rang $n$ et $\beta$ symétrique. 


\section{Définition 1.3}

Soit $(M, \beta)$ un module bilinéaire. Le déterminant de $\beta$ noté $\operatorname{det} \beta$ est le déterminant de $M_{\beta}$. La proposition précédente montre que det $\beta$ est défini modulo $A^{*^{2}}$.

Le discriminant de $\beta$ noté discr $\beta=(-1)^{\frac{n i n-1)}{2}} \operatorname{det} \beta$.

\section{Définitions 1.4}

Soit $N$ un sous- $A$-modulc de $M$ muni de la forme bilinéaire $\beta$.

On note $N^{\perp}$ pour $\{x \in M \mid \beta(x, y)=0 \forall y \in N\}$.

Il est clair que $(M, \beta)$ est non dégénéré si et seulement si $M^{\perp}=\{0\}$, car $M^{\perp}$ est le noyau de $f_{\beta}$.

Soient $N$ et $N^{\prime}$ deux sous- $A$-modules de $M$ tels que $N \cap N^{\prime}=\{0\} . N \oplus N^{\prime}$ se note $N \boxplus N^{\prime}$ si $N^{\prime} \subset N^{\perp}$.

\section{Proposition 1.5}

Soient $(M, \beta)$ un $A$-module bilinéaire non dégénéré et $N$ un sous-module de $M$, tel que $\left.\beta\right|_{N}$ soit non dégénérée. Alors $M=N \boxplus N^{-}$.

\section{Démonstration :}

Il suffit de voir que $M=N \oplus N$.

Soit $x \in M$, posons $f=\left.f_{\beta}(x)\right|_{N}$. On a $f \in N^{*}$; or par hypothèse, $\left.f_{\beta}\right|_{N}$ est un isomorphisme. Il existe donc $y \in N$ tel que $\left.\int_{\beta}\right|_{N^{*}}(y)=f$. On a ainsi :

$$
\beta(x, z)=f_{\beta}(x)(z)=f(z)=f_{\left.\beta\right|_{N}}(y)(z)=\beta(y, z) \quad \forall z \in N .
$$

Donc $\beta(x-y, z)=0 \forall z \in N$ ce qui nous donne $x-y \in N^{\perp}$.

Finalement, on a $x=y+(x-y) \in N \boxplus N^{2}$. Le fait que $N \cap N^{\perp}=\{0\}$ est trivial.

\section{Corollaire 1.6}

Si $A$ est un corps de caractéristique différente de 2 , alors $\beta \simeq \beta^{\prime}$ où $\beta^{\prime}$ est une forme diagonale, c'est-à-dire que la matrice $M_{\beta}$ est diagonale et on la note $\left\langle a_{1}, \ldots, a_{2}\right\rangle$, les $a_{i}$ étant les coefficients diagonaux de $M_{\beta}$.

\section{Démonstration :}

S'il existe $x$ et $y$ tels que $\beta(x, y) \neq 0$, alors $\beta(x, x), \beta(y, y)$ ou $\beta(x+y, x+y)$ est non nul. Supposons que ce soit $x ;\left.\beta\right|_{<x\rangle}$ est donc non dégénérée, par la proposition précédente. On a que $\beta \simeq\langle x\rangle \boxplus\langle x\rangle^{\perp}$, et on termine par récurrence.

\section{Définitions 1.7}

Soient $A$ un anneau commutatif et $M$ un $A$-module. Une forme quadratique est une application:

$$
q: M \longrightarrow A \text { tolle que } q(\lambda x)=\lambda^{2} q(x) \quad \forall \lambda \in A \text { et } x \in V^{\prime}
$$

et telle que l'application $\beta_{q}$ avec

$$
\beta_{q}(x, y)=q(x+y)-q(x)-q(y) \quad \forall x, y \in M
$$

soit bilinéaire symétrique.

On dit que $q$ est non dégénérée si $\beta_{q}$ est non dégénérée, de même $\operatorname{det} q=\operatorname{det} \beta_{q}$ et $\operatorname{discr} q=\operatorname{discr} \beta_{q}$.

On dit alors que $(M, q)$ est un module quadralique.

Si $M=N \boxplus N^{\prime}$ pour $\beta_{q}$, alors $q\left(N \boxplus N^{\prime}\right)=q(N)+q\left(N^{\prime}\right)$. On peut donc aussi écrire $M=N \boxplus N^{\prime}$ pour $q$. 


\section{Remarque :}

Soit $(M, \beta)$ un module bilinéaire. Il est très simple de transformer $M$ en module quadratique, il suffit de prendre $q: M \longrightarrow A$ définie par $x \longmapsto \beta(x, x)$. Il faut noter que dans ce cas $\operatorname{det} q=2^{n} \operatorname{det} \beta$ où $n$ est la dimension de $M$, car $\beta_{q}=2 \beta$.

Réciproquement, si $(M, q)$ est un module quadratique, $\beta_{q}$ cst entièrement déterminé par $q$, donc $\left(M, \beta_{q}\right)$ est clairement un module bilinéaire.

Mais attention, il serait faux de croire qu'il y a une correspondance bi-univoque entre les deux notions : si cela est vrai sur les corps de caractéristiques différentes de 2 , cela n'est pas vrai sur $\mathbb{Z}$ ni sur $\mathbb{F}_{2}$, ni sur les anneaux $p$-adiques et encore moins sur $2 / 8$. Par exemple sur $\mathbb{Z}$, la forme quadratique $x_{1}^{2}+x_{1} x_{2}+x_{2}^{2}$ ne peut jamais s'écrire $\beta(x, x)$ où $\beta$ est une forme bilinéaire; d'autre part, sur $\mathbb{F}_{2}$, toute forme bilinéaire $\beta_{q}$ associée à une forme quadratique $q$ est "alternée" (i.e. $\beta_{q}(x, x)=0 \forall x$ ) donc n'est pas représentative de toutes les formes bilinéaires sur $\mathbb{F}_{2}$.

De plus on ne peut pas affirmer que l'une est plus "pratique" que l'autre : s'il est vrai qu'on peut facilement utiliser l'interprétation matricielle pour les formes bilinéaires, le théorème de Witt (voir chapitres 3 et 4) n'est pas toujours vrai pour elles, alors qu'il l'est pour toute forme quadratique non dégénérée.

\section{B. Anneaux et corps p-adiques}

Dans ce paragraphe, nous ferons une présentation succincte des anneaux $\mathbb{Z}_{p}$ et des corps $\mathbb{Q}_{p}$. Il me semble qu'il n'y a pas de manière plus courte et élégante de définir ces ensembles que celle de J.P. Serre dans [10, pp. 23-26], c'est pourquoi je ne donnerai que les résultats sans rien démontrer.

\section{Définition 1.8}

Soient $n \in I\}, n \geq 2, p$ premier ot $\varphi_{n}$ l'homomorphisme naturel de $Z / p^{n} Z$ dans $\mathbb{Z} / p^{n-1} \mathbb{Z}$ qui est évidemment surjectif.

On définit alors

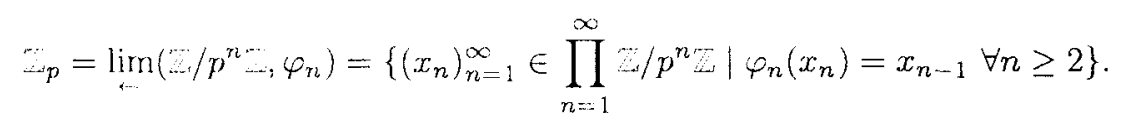

L'addition, la multiplication et la topologie sur $\mathbb{Z}_{p}$ sont héritées de celles induites par l'anneau topologique produit $\prod_{n=1}^{\infty} \mathbb{Z} / p^{n} \Xi$. Les anneaux $\Xi / p^{n}$ ítant munis de la topologie discrète, nous avons donc que $\prod_{n=1}^{\infty} \mathbb{Z} / p^{n} \mathbb{Z}$ est compact (Tychonov), donc $\mathbb{Z}_{p}$ aussi puisqu’il est fermé.

\section{Théorème 1.9}

$\mathbb{Z}_{p}$ possède les propriétés suivantes :

(I) $\mathbb{Z}_{p} / p^{n} \mathbb{Z}_{p}=\mathbb{Z} / p^{n} \mathbb{Z}$

(II) $\mathbb{Z}_{p}$ est un anneau local d’idéal maximal $p^{\mathbb{Z}} p$, donc les seuls idéaux de $\mathbb{Z}_{p}$ sont les $p^{n} \mathbb{Z}_{p}, n \in \mathbb{N} ;$ il suit que

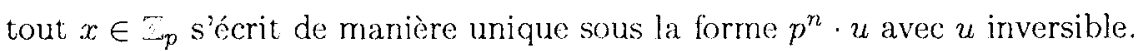

(III) L'application

$$
\begin{aligned}
& v_{p}: \cdots p \longrightarrow\lfloor\cup\{\infty\} \\
& x \longmapsto n \text { tel que } x=p^{n} \cdot u \\
& 0 \longmapsto \infty
\end{aligned}
$$

est appetée valualion $p$-adique. Hille induit une distance $: d(x, y)=p^{-v_{p}(x-y)}$ qui définit la topologie de $\mathbb{Z}_{p}$. On a en outre que $\mathbb{Z}$ est dense dans $\mathscr{E}_{p}$ qui est complet.

$(I V) \mathbb{Z}_{p} \cap \mathbb{Q}=\mathbb{Z}_{(p)}=\left\{\frac{a}{b} \in \mathbb{O} \mid p \not b\right\}$ 


\section{Définition 1.10}

Notons $\mathbb{Q}_{p}$ le corps des fractions de $\mathbb{Z}_{p}$. Vu ce qui précède, on a bien sûr que $\mathbb{Q}_{p}=\mathbb{Z}_{p}\left[p^{-1}\right]$, donc tout $x \in Q_{p}$ s'écrit aussi de manière unique sous la forme $p^{n} \cdot u$, où $u$ est un inversible de $\mathbb{Z}_{p}$ mais maintenant, $n \in \mathbb{Z} ; n$ s'appellera aussi valuation $p$-adique que l'on notera aussi $v_{p}(x)$; elle induira de la même manière la topologie sur $\mathbb{Q}_{p}$, et on obtient facilement le théorème suivant.

\section{Théorème 1.11}

(I) Le corps $Q_{p}$, muni de la distance $d(x, y)=p^{-v_{p}(x-y)}$ est localement compact et complet; le corps $\mathbb{Q}$ est dense dans $\mathbb{Q}_{p}$.

(II) La distance $d$ est "ultramétrique", c'est-à-dire qu'elle vérifie l'inégalité suivante :

$$
d(x, y) \leq \max (d(x ; z), d(z, y))
$$

Nous obtenons grâce à cela le fait agréable que toute séric de $\varsigma_{p}$ ou de $\Xi_{p}$ est convergente si et seulement si son terme général tend vers 0 .

\section{Remarque :}

Nous aurions pu définir $\mathbb{Q}$, de manière tout à fait analytique, comme le complété de $\mathbb{Q}$ pour la distance $d$, en voyant $\mathbb{Z}_{p}$ comme la boule unité et $p \mathbb{Z}_{p}$ comme la boule unité privée de la sphère unité.

\section{Réseaux et bases de réseaux}

Nous allons donner dans ce paragraphe un critère pour pouvoir compléter des vecteurs linéairement indépendants en une base de réseau.

\section{Définitions 1.12}

Soient $A$ un anneau principal, (dans la pratique $A$ sera où $Z_{p}$ ), $K$ son corps des fractions, $V$ un $K$ espace vectoriel de dimension $n$ et $\left(e_{1}, \ldots, c_{n}\right)$ uno $K$-base de $V$. Alors l'ensemble des $\lambda_{1} e_{1}+\cdots+\lambda_{n} e_{n}$ où les $\lambda_{i}$ parcourent $A$ est appclé $A$-réseau et $\left(e_{1}, \ldots, e_{n}\right)$ est appelé base du réseau.

Soit $\Lambda$ un réseau. Un vecteur de $\Lambda$ est dit primilif, siil est possible de trouver $n-1$ dutres vecteurs formant avec lui une base de $\Lambda$.

Si $\Lambda \subset \Gamma$ sont deux $A$-réseaux, on dit que $\Lambda$ est un sous-A-réseau de $\Gamma$.

\section{Remarque :}

Il existe une définition plus générale si l'anneau n'est pas principal, mais nous n'en n'aurons pas besoin.

\section{Définition 1.13}

Soient $\Gamma \subset \Lambda$ deux $A$-réseaux munis des bases $\left(b_{1}, \ldots, b_{n}\right)$ et $\left(e_{1}, \ldots, e_{n}\right)$ respectivement. Pour tout $i, j \in \mathbb{N}_{n}$, il existe $r_{i j} \in K$ tel que $b_{2}=\sum_{j=1}^{n} r_{i j} c_{j}$.

$d(\mathrm{\Gamma} / \Lambda) \stackrel{\text { def }}{=} d e l\left(r_{i j}\right)$ est appelé le discriminant de $\mathrm{\Gamma}$ sur $\Lambda$.

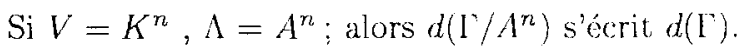




\section{Remarque :}

Le discriminant est unique à un facteur de $U(A)$ près.

\section{Démonstration :}

Soient $\left(e_{1}, \ldots, e_{n}\right),\left(e_{1}^{\prime}, \ldots, e_{n}^{\prime}\right)$ deux bases d'un $A$-réseau $\Lambda$, et soient $\left(b_{1}, \ldots, b_{n}\right),\left(b_{1}^{\prime}, \ldots, b_{n}^{\prime}\right)$ deux bases d'un sous- $A$-réseau $\Gamma$ de $\Lambda$.

On a que $b_{i}=\sum_{j=1}^{n} r_{i j} e_{j}, b_{i}^{\prime}=\sum_{j=1}^{n} r_{i j}^{\prime} e_{j}^{\prime}$ avec $r_{i j}, r_{i j}^{\prime} \in A$ et $b_{i}=\sum_{j=1}^{n} \alpha_{i j} b_{j}^{\prime}, e_{i}^{\prime}=\sum_{j=1}^{n} \beta_{i j} e_{j}$ avec $\left(\alpha_{i j}\right)_{i, j \in N_{n}}=C$ et $\left(\beta_{i, j}\right)_{i, j \in N_{n}}=B \in C l_{n}(A)$.

On trouve facilement que

$$
C\left(r_{i j}^{\prime}\right)_{i, j \in \mathbb{N}_{n}} B=\left(r_{i j}\right)_{i, j \in \mathbb{N}_{n}}
$$

et on conclut en considérant le fait que toute matrice de $G l_{n}(A)$ a pour déterminant un élément de $U(A)$.

\section{Lemme 1.14}

Soit $\Gamma$ un sous- $A$-réseau de $\Lambda$. Alors

$$
d(\mathrm{~T} / \Lambda) \Lambda \subset \Gamma .
$$

\section{Démonstration :}

Il suffit de montrer ce fait pour les éléments d'une base $\left(e_{1}, \ldots, e_{n}\right)$ de $\Lambda$. Soit $\left(b_{1}, \ldots, b_{n}\right)$ une base de $\Gamma$. Par définition de $\Gamma$, il existe $\left(\gamma_{i j}\right)_{i, j \in \aleph_{n}} \in M_{n}(A)$ telle que $d(\Gamma / \Lambda)=\operatorname{det}\left(\gamma_{i j}\right)$ et $\sum_{j=1}^{n} \gamma_{i j} e_{j}=b_{j}$.

En résolvant ce système, on trouve une matrice $\left(\gamma_{i j}^{\prime}\right)_{i, j \in \mathbb{N}_{n}} \in M_{n}(A)$ telle que $d(\Gamma / \Lambda) e_{i}=\sum_{j=1}^{n} \gamma_{i j}^{\prime} b_{j} \in \Gamma$.

\section{Lemme 1.15}

Soit $\Gamma$ un sous- $A$-réseau de $\Lambda$ cu $\left(c_{1}, \ldots, c_{n}\right)$ une base de $\Lambda$. Alors il existe $\left(a_{1}, \ldots, a_{n}\right)$ une base de $\Gamma$ telle que :

avec

$$
\begin{aligned}
a_{1} & =s_{11} e_{1} \\
a_{2} & =s_{12} e_{1}+s_{22} e_{2} \\
\vdots & \\
a_{n} & =s_{n 1} e_{1}+\cdots+s_{n n} e_{n}
\end{aligned}
$$

\section{Dérnonstration :}

$$
s_{i j} \in A \text { et } s_{i i} \neq 0, i, j \in \mathbb{N}_{n}
$$

Posons $\Gamma^{(j)}=\left\{a \in \Gamma \mid a=\sum_{i=1}^{j} \gamma_{i} e_{i}\right\}$ et $P_{j}=\left\{\gamma_{j} \mid \exists \gamma_{1}, \ldots, \gamma_{j-1}\right.$ avec $\left.\sum_{i=1}^{j} \gamma_{i} e_{i} \in \Gamma^{(j)}\right\} . P_{j}$ est un idéal non nul de $A$. En effet, $d(\Gamma / \Lambda) e_{j} \in \Gamma^{(j)}$, et si $\mu, \nu \in A$ et $\gamma_{j}, \gamma_{j}^{\prime} \in P_{j}$ alors $\mu \gamma_{j}+\nu \gamma_{j}^{\prime} \in P_{j}$ clairement.

Puisque $A$ est principal, il existe $s_{j j} \neq 0$ tel que $P_{j}=s_{j j} A$, et par définition de $P_{j}$, on pourra trouver $s_{1 j}, \ldots, s_{j-1 j}$ tels que $a_{j}:=\sum_{i=1}^{j} s_{i j} e_{i} \in \Gamma^{(j)}$ pour tout $j \in \|_{n}$.

Montrons que les $a_{j}$ engendrent $\Gamma$ :

Soit $a=\sum_{i=1}^{n} \mu_{i} e_{i} \in \Gamma=\Gamma^{(n)}$ avec $\mu_{n} \in P_{n}=s_{n n h}$ A. Donc,

$$
a-\nu_{n} a_{n}=\sum_{i=1}^{n-1} \mu_{i}^{\prime} e_{2} \in \Gamma^{(n-1)}
$$

et, par itération du procédé on trouve que $a=\sum_{i=1}^{n} \nu_{n} a_{n}$. 


\section{Lemme 1.16}

Soient $c_{1}, \ldots, c_{p}$ des vecteurs linéairement indépendants d'un $A$-réseau $\Lambda$. Alors il existe une base $\left(b_{1}, \ldots, b_{n}\right)$ de $\Lambda$ telle que :

$$
\begin{aligned}
& c_{1}=s_{11} b_{1} \\
& c_{2}=s_{12} b_{1}+s_{22} b_{2} \\
& \vdots \\
& c_{p}=s_{n 1} b_{1}+\cdots+s_{p p} b_{p}
\end{aligned}
$$

avec

$$
s_{i j} \in A \text { et } s_{i i} \neq 0, i, j \in \mathrm{IJ}_{p}
$$

\section{Démonstration :}

On peut choisir $c_{p+1}, \ldots, c_{n} \in \Lambda$ tels que $c_{1}, \ldots, c_{n}$ soient linéairement indépendants. Posons $\Gamma$ le sousréseau de $\Lambda$ engendré par $c_{1}, \ldots, c_{n}$. Par le lemme 1.14 , on a $d \Lambda \subset \Gamma$ où $d=d(\Gamma / \Lambda)$. Grâce au lemme précédent, nous pouvons trouver $\left(b_{1}, \ldots, b_{n}\right)$ une base de $\Lambda$ telle que :

$$
\begin{aligned}
& d b_{1}=t_{11} c_{1} \\
& d b_{2}=t_{12} c_{1}+t_{22} c_{2} \\
& \quad \vdots \\
& d b_{n}=t_{n 1} c_{1}+\cdots+t_{n n} c_{n}
\end{aligned}
$$

avec

$$
t_{i, j} \in A \text { et } t_{i i} \neq 0, i, j \in 1_{n} .
$$

En résolvant le système par rapport aux $c_{i}$, nous obtenons un système du type cherché. A priori, les $s_{i j}$ se trouvent dans $K$ seulement. Cependant, les $b_{i}$ forment une base de $\Lambda$ (et de $V$ ), puis $c_{i} \in \Lambda$; done on trouve que $s_{i j} \in A$ grâce à l'unicitć de l'écriture de tout élément relativement à une base. De plus, $s_{i i}=d / t_{i i} \neq 0$.

\section{Théorème 1.17}

Soient $j \leq n \in \mathrm{I}, A$ un anneau principal et intègre, $K$ son corps des fractions et $c_{1}, \ldots, c_{j} \in A^{n}$ linéairement indépendants. Les affirmations suivantes sont équivalentes:

i) Il existe $c_{j+1}, \ldots, c_{n}$ tels que $c_{1}, \ldots, c_{n}$ soit une base de $A^{n}$.

ii) Les sous-déterminants de rang $j$ de la matrice $n \times j\left(c_{1} c_{2} \cdots c_{j}\right)$ n'ont pas de diviseurs communs.

iii) Si $a=v_{1} c_{1}+\cdots+v_{j} c_{j} \in \Lambda^{n}$ avec $v_{1}, \ldots, v_{j} \in K$, alors $v_{1}, \ldots, v_{j} \in A$.

\section{Démonstration :}

i) $\Rightarrow$ ii):

Soit $\left(c_{1}, \ldots, c_{n}\right)=P \in M_{n}(A)$. On a $\operatorname{det}(P) \in U(A)$. Par le développement de Laplace à partir des $j$ premières colonnes, on a $: \operatorname{det}(P)=\sum R_{M} \cdot R_{M^{\prime}} \in U(A)$ où les $R_{M}$ sont les déterminants des matrices $j \times j$ en parcourant les $j$ premières colonnes de $P$ et où les $R_{M^{\prime}}$ sont les déterminants des matrices $(n-j) \times(n-j)$ "complémentaires". Et par le théorème de Bezout, on conclut.

ii) $\Rightarrow$ iii):

Soit $a=v_{1} c_{1}+\cdots+v_{j} c_{j} \in A^{n}$. Il existe $w_{1}, \ldots, w_{n} \in A$ tels que $a=w_{1} e_{1}+\cdots+w_{n} e_{n}$ où $\left(e_{1}, \ldots, e_{n}\right)$ est la base canonique de $A^{n}$, donc

$$
w_{i}=\sum_{k=1}^{j} v_{k} c_{i k} \forall i \in I_{n} \quad Q_{i}
$$


avec $c_{i}=\sum_{k=1}^{n} c_{k i} e_{k}$, ò̀ $c_{i k} \in A$ pour tout $i \in N_{n}, k \in \mathrm{H}_{j}$.

En prenant au hasard $j$ équations de type $\nabla_{i}$ et en résolvant par rapport à $v_{k}$, on obtient que $v_{k} R_{M} \in A$ pour tout $k \in N_{j}$ et pour toute sous-matrice $M$ de rang $j$ de la matrice $\left(c_{1} \cdots c_{j}\right)$. Or, par hypothèse, et par le théorème de Bezout, il existe $\lambda_{1}, \ldots, \lambda_{m} \in A$ tels que $\sum_{M} \lambda_{i} R_{M}=1$ donc $v_{k}=\sum_{M} \lambda_{i}\left(R_{M} v_{k}\right) \in A$ pour tout $k \in \mathrm{I}_{j}$.

iii) $\Rightarrow$ i) :

Grâce au lemme précédent, on peut trouver $\left(b_{1}, \ldots, b_{n}\right)$ une base de $A^{n}$ telle que :

$$
\begin{aligned}
& c_{1}=s_{11} b_{1} \\
& c_{2}=s_{12} b_{1}+s_{22} b_{2} \\
& \vdots \\
& c_{j}=s_{n 1} b_{1}+\cdots+s_{j j} b_{p}
\end{aligned}
$$

avec $s_{i j} \in A$ et $s_{i i} \neq 0$. En résolvant, on trouve que :

$$
\begin{aligned}
& b_{1}=s_{11}^{\prime} c_{1} \\
& b_{2}=s_{12}^{\prime} c_{1}+s_{22}^{\prime} c_{2} \\
& \vdots \\
& b_{j}=s_{n 1}^{\prime} c_{1}+\cdots+s_{j j}^{\prime} c_{p}
\end{aligned}
$$

avec $s_{i j}^{\prime} \in K$ pour tout $i, j$. Or $b_{1}, \ldots, b_{j} \in A^{n}$, donc par hypothèse $s_{i j}^{\prime} \in A \quad \forall i, j$. Finalement, $\left(c_{1}, \ldots, c_{j}, b_{j: 1}, \ldots, b_{n}\right)$ est une base de $A^{n}$.

\section{Corollaire 1.18}

$\left(x_{1}, \ldots, x_{n}\right) \in A^{n}$ est primitif $\Longleftrightarrow x_{1}, \ldots, x_{n}$ ne possèdent pas de diviseurs communs.

\section{Réseaux bilinéaires et quadratiques}

\section{Définitions 1.19}

Soient $A$ un anneau principal, $K$ son corps des fractions et $(V, \beta)$ un $K$-espace vectoriel bilinéaire.

Soit $\Lambda$ un $A$-réseau; on dit que $\Lambda$ est un réseau bilinéaire si $\beta(x, y) \in A \quad \forall x, y \in \Lambda$.

Les réseaux quadratiques se définissent de la mêmo manière.

\section{Remarque :}

Si $M$ est un $A$-module bilinéaire libre de rang $n, M$ peut être vu comme $A$-réseau bilinéaire sur $V=$ $M \otimes_{A} K$.

\section{Définition 1.20}

Soient $A$ et $K$ comme dans la défirition 1.19, et soit $(V, q)$ un $K$-espace vectoriel quadratique. Pour tout $A$-réseau $\Lambda$ de $V$, on définit $\Lambda^{\#}=\left\{x \in V \mid \beta_{q}(x, y) \in A \quad \forall y \in \Lambda\right\}$.

Si $\Lambda$ est un réseau quadratique, il est clair que $\Lambda \subset \Lambda^{\# *}$. 


\section{Proposition 1.21}

Si $\Lambda$ est un réseau d'un $K$ espace vectoriel quadratique non dégénéré $(V, q)$ de dimension $n$, alors $\Lambda^{\#}$ est aussi un réseau, et si $\Lambda$ est un réseau quadratique et q est non dégénérée sur $\Lambda$, alors $\Lambda=\Lambda^{\#}$.

\section{Démonstration :}

On sait par hypothèse que

$$
\begin{aligned}
\int_{\beta_{q}}: V & \longrightarrow \operatorname{Hom}_{K}(V, K) \\
x & \longmapsto \beta_{q}(x, \cdot)
\end{aligned}
$$

est un isomorphisme. Soit $\mathfrak{B}=\left(e_{1}, \ldots, e_{n}\right)$ une $A$-base de $\Lambda$. On sait que $\operatorname{Hom}_{K}(V, K)$ est engendré par les $e_{i}^{\# \#}$ où $e_{i}^{\#}\left(e_{j}\right)=\delta_{i j}, \quad \forall i, j \in \|_{n}$. $\|$ cxiste donc des $c_{i}$ linéairement indépendants tels que $f_{\beta_{q}}\left(c_{i}\right)=$ $e_{i}^{\#}, \quad \forall i$. Nous allons voir que $\Lambda^{\#}=\sum_{i=1}^{n} A c_{i}$.

Le fait que $\Lambda^{\#} \supset \sum_{i=1}^{n} A c_{i}$ découle directement de la définition des $c_{i}$.

Soit maintenant $x \in \Lambda^{\#}$. On a $\beta_{q}\left(x, e_{1}\right)=\lambda_{1} \in A$. Or $\lambda_{1}=\beta_{q}\left(\lambda_{1} c_{1}, e_{1}\right)$, donc $\beta_{q}\left(x-\lambda_{1} c_{1}, e_{1}\right)=0$.

On a aussi $\beta_{q}\left(x-\lambda_{1} c_{1}, e_{2}\right)=\beta_{q}\left(\lambda_{2} c_{2}, e_{2}\right) \in A$ car $c_{i} \in \Lambda^{\#} \forall i$. Done $\beta_{q}\left(x-\lambda_{1} c_{1}-\lambda_{2} c_{2}, e_{2}\right)=0$, de même $\beta_{q}\left(x-\lambda_{1} c_{1}-\lambda_{2} c_{2}, e_{1}\right)=0$ par définition des $c_{i}$. On recommence alors ce procédé, et on obtient que $\beta_{q}\left(x-\sum \lambda_{i} c_{i}, e_{j}\right)=0 \forall j$ donc $x=\sum \lambda_{i} c_{i}$.

Supposons maintenant que $\Lambda$ soit un réseau quadratique, donc que $\Lambda \subset \Lambda^{\#}$. $\mathfrak{B}$ étant une base de $V$, on a que $c_{i}=\sum_{i=1}^{n} \lambda_{i j} e_{j} \forall i \in l_{n}$ avec $\lambda_{i j} \in K$. Par le choix des $c_{i}$, on a :

$$
\delta_{i k}=\beta_{q}\left(c_{i}, c_{k}\right)=\sum_{j=1}^{n} \lambda_{i j} \beta_{q}\left(e_{j}, \epsilon_{k}\right)
$$

ce qui nous donne : $L \cdot B=I_{n}$ ò̀ $L_{2 j}=\lambda_{i j} \forall i, j$, donc $L=B^{-1}$. Puisque $q$ est non dégénérée sur $\Lambda$, on a que $\operatorname{det} B$ est inversible dans $A$, donc $L$ est à coeflicient dans $A$. D'où $\Lambda=\Lambda^{\#}$.

\section{Définitions 1.22}

Soient $A$ un anneau commutatif et $(M, \beta)$ un $A$-module bilinéaire.

On définit $O_{\beta}(M)=\{u: M \longrightarrow M \mid u$ est un isomorphisme et $\beta(u(x), u(y))=\beta(x, y) \forall x, y \in M\}$.

La composition des applications munit naturellement cet ensemble d'une structure de groupe, et on l'appellera groupe orthogonal de $M$.

$\mathrm{Si}(M, q)$ est un $A$-module quadratique, on définil de même

$O_{q}(M)=\{u: M \longrightarrow M \mid u$ est un isomorphisme et $q(u(x))=q(x) \forall x \in M\}$.

\section{Remarque importante :}

Soient $A$ un anneau principal, $K$ son corps des fractions, $(V, \beta)$ un espace vectoriel bilinéaire et $M, M^{\prime}$ deux réseaux bilinéaires.

Supposons que $\left(M,\left.\beta\right|_{M}\right) \cong\left(M^{\prime},\left.\beta\right|_{M^{\prime}}\right)$ cn lant que A-modules bilinéaires. Il existe donc un isomorphisme $u: M \longrightarrow M^{\prime}$ tel que $\beta_{M}(x, y)=\beta_{M^{\prime}}(u(x), u(y)) \forall x, y \in M$. Puisque $M$ et $M^{\prime}$ contiennent des bases de $V, u$ se prolonge en $u \in O_{3}(V)$

Inversément, si $u \in O_{B}(V)$ et $M$ est un $A$-réscau bilinćaire, $M^{\prime}=u(M)$ est aussi un $A$-réseau bilinéaire et on a bien sûr que $\left(M,\left.\beta\right|_{M}\right) \stackrel{\subseteq}{\simeq}\left(M^{\prime},\left.\beta\right|_{M^{\prime}}\right)$ en tant que $A$-modules bilinéaires.

Soient maintenant $u_{1}, u_{2} \in O_{3}(V)$ et $M$ comme avant; supposons que $u_{1}(M)=u_{2}(M)$, c'est-à-dire $\left.u_{1}^{-1} u_{2} \in O_{\beta}\right|_{M}(M)$. On obtient donc la proposition suivante:

\section{Proposition 1.23}

Soient comme avant $A, K,(V, \beta)$ et $M$ un réseau bilinćaire. Alors $O_{\beta}(M) \subset O_{\beta}(V)$ canoniquement, et il y a bijection entre l'ensemblo des sous- $A$-réseaux bilinéaires de $(V, \beta)$ qui sont isomorphes à $M$ en tant que $A$-modules bilinéaires et les classes de $O_{B}(V)$ à gauche de $O_{\beta}(M)$. 


\section{E. Quelques rappels}

Nous citerons dans ce paragraphe des résultats classiques sur les formes bilinéaires et quadratiques entières, entre autres le théorème de finitude et le théorème de Hasse-Minkowski.

\section{Définition 1.24}

Soit $(M, \beta)$ un $\mathbb{Z}$ module bilinéaire libre de rang $n .(M, \beta)$ est dit de type $(I I)$ si $\beta(x, x)$ est pair pour tout $x \in M ;$ si $(M, \beta)$ n'est pas de type $(I I)$ il est de type $(I)$.

On note $\mathscr{S}_{n}$ la catégorie des $\cdots$-modules bilinćaires libres de rang $n$ non dégénérés et définis positifs. $\mathscr{C}_{n}$ est l'ensemble des classes d'isomorphismes de $\mathscr{F}_{n}$ qui sont de type $(I I)$.

Finalement, on note $\mathscr{Z}_{n}$ l'ensemble des classes d'isomorphismes de $\mathscr{S}_{n}$ qui sont de type $(I)$.

\section{Théorème 1.25 (théorème de finitude)}

Le cardinal des classes à isomorphismos près des ćlóments de $\mathscr{S}_{n}$ est fini, ou plus généralement, le cardinal des classes d'équivalences des formes bilinéaires entières de déterminant $d$ donné est fini.

\section{Démonstration :}

Une démonstration de ce théorème est donnée dans [3, pp. 135-137].

\section{Corollaire 1.26}

$\mathscr{C}_{n}$ et $\mathscr{H}_{n}$ sont finis.

\section{Corollaire 1.27}

Le théorème 1.25 et le corollaire 1.26 sont aussi vrais si les modules considérés sont quadratiques.

\section{Démonstration :}

$(M, q) \simeq\left(M^{\prime}, q^{\prime}\right) \Longleftrightarrow\left(M, \beta_{q^{\prime}}\right) \simeq\left(M^{\prime}, \beta_{q^{\prime}}\right)$ car $\ldots$ est intègre.

\section{Définition 1.28}

Soit $(M, \beta)$ un $\cdots$-module bilinciaire libre do rang $n$. Alors $M \otimes-\cdots$, , est un $\cdots$-module bilinéaire libre de rang $n$ que l'on note $\left(M_{p}, \beta_{p}\right)$.

De même, si $(V, \beta)$ est un espace bilinéaire de dimension $n$; en tensorisant par $\mathbb{C}_{p}$, on obtient $\left(V_{p}, \beta_{p}\right)$ et en tensorisant par $\mathcal{E}_{-}$, on obtient $\left(V_{\infty}, \beta_{\infty}\right)$.

On peut bien sûr faire de mêrne avec des espaces quadratiques.

\section{Définition 1.29}

Soient, l'ensemble des nombres premiers positifs et ${ }^{\prime \prime}=U\{\infty\}$; par convention, $c_{\infty}=\mathbb{P}$. Fixons-nous $p \in \mathbb{I}^{\prime}$. Pour lout $a$ et $b \in a_{-}^{*}$; on pose :

$$
(a, b)_{p}= \begin{cases}1 & \text { si } a x^{2}+b y^{2}=z^{2} \text { possède une solution non triviale dans } Q_{p} \\ -1 & \text { sinon. }\end{cases}
$$

Ce nombre s'appelle le symbole de Hilbert de a et b.

\section{Proposition 1.30}

Soient $p \in \mathbb{P}^{\prime \prime}, a, a^{\prime}, b, c \in a_{p}^{*}$ et $d \in a_{p}^{*} \backslash\{1\}$. Les égalités suivantes sont satisfaites :

i) $(a, b)_{p}=(b, a)_{p}$

ii) $\left(a a^{\prime}, b\right)_{p}=(a, b)_{p}\left(a^{\prime}, b\right)_{p}$

iii) $(c,-c)_{p}=(d, 1-d)_{p}=1$. 


\section{Théorèrne 1.31}

On a les égalités :

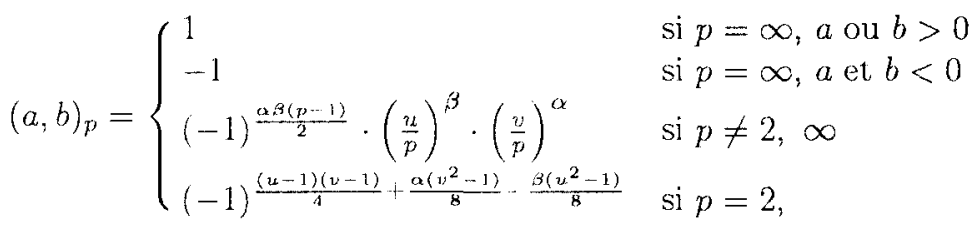

où $(\dot{\bar{p}})$ est le symbole de Legendre et $a$ respectivement $b$ valent $p^{\alpha} u$ et $p^{\beta} v, u$ et $v$ étant des unités de $\mathbb{z}_{p}$.

Théorème 1.32 (Formule du produit de Hilbert)

Soient $a, b \in 0^{*}$. Alors $(a, b)_{p}=1$ sauf sur un sous-ensemble fini de in' et

$$
\prod_{p<}(a, b)_{p}=1
$$

\section{Démonstration :}

Ces résultats sur le symbole de Hilbert sont démontrés dans [10, pp. 37-45] •

\section{Théorème 1.33 (Hasse-Minkowski)}

Soient $(V, \beta)$ et $\left(V^{\prime}, \beta^{\prime}\right)$ deux 0 -espaces bilinéaires de dimension $n$. Alors :

$$
(V, \beta) \stackrel{\square}{\simeq}\left(V^{\prime}, \beta^{\prime}\right) \text { si et sculement si }\left(V_{p}, \beta_{p}\right) \stackrel{\mathscr{C H}^{\prime}}{\simeq}\left(V_{p}^{\prime}, \beta_{p}^{\prime}\right) \quad \forall p \in \mathbb{F}^{\prime}
$$

\section{Démonstration :}

Ce théorème hauternent non trivial, utilise ce que l'on vient de voir sur le symbole de Hilbert et demande une connaissance approfondie des formes bilinéaires sur les corps p-adiques. Toute la première partie de [10] est consacrée à la démonstration de ce théorèrne.

\section{Remarque :}

Ce résultat est aussi vrai pour des espaces quadratiques, puisque tous ces corps sont de caractéristique nulle (y compris les p-adiques, bion que leur nom pourrail nous faire présupposer autre chose ...)

\section{F. La notion de genre}

Dans ce paragraphe, nous introduirons une nouvelle relation d'équivalence sur les modules bilinéaires; nous verrons que pour ceux qui sont libres de rang $n$ et définis positifs, il $\mathrm{n}$ y a que deux classes d'équivalence : $\mathscr{H}_{n}$ et $\mathscr{C}_{n}$.

\section{Définition 1.34}

Soient $(M, \beta)$ et $\left(M^{\prime}, \beta^{\prime}\right)$ deux $\cdots-$ modules bilinéaires libres de rang $n$, on dit que $(M, \beta)$ est dans le même genre que $\left(M^{\prime}, \beta^{\prime}\right)$ si

$$
\left(M_{p}, \beta_{p}\right) \stackrel{\sim}{\simeq}\left(M_{p}^{\prime}, \beta_{p}^{\prime}\right) \quad \forall p \in \mathbb{F ^ { \prime }}
$$

avec la convention que $\bar{\Xi}_{\infty}=$ F. Et on écrit $(M, \beta) \sim\left(M^{\prime}, \beta^{\prime}\right)$.

On définit cette notion de manière identique pour les modules quadratiques. 


\section{Remarque :}

Si $(M, \beta) \simeq\left(M^{\prime}, \beta^{\prime}\right)$ alors $(M, \beta) \sim\left(M^{\prime}, \beta^{\prime}\right)$. Mais la réciproque n'est pas vraie, par exemple en dimension 2 , où les formes $\beta$ et $\beta^{\prime}$ définies par les matrices $\left(\begin{array}{cc}2 & 0 \\ 0 & 17\end{array}\right)$ et $\left(\begin{array}{cc}1 & 0 \\ 0 & 34\end{array}\right)$ sont dans le même genre mais elles ne sont pas E-équivalentes. Cet excrnple illustre bien qu'il n'y a pas d'équivalent au théorème de Hasse-Minkowski pour les formes entières.

Tout d'abord, nous allons énoncer toute une série de rísultats :

\section{Théorème 1.35}

Soient $f \in \mathbb{Z}_{p}\left[X_{1}, \ldots, X_{m}\right], x \in\left(\mathbb{Z}_{p}\right)^{m}, n, k \in\left[!\right.$ et $j \in ! !_{m}$.

Supposons que

$$
0 \leq 2 k<n, \quad f(x) \equiv 0 \quad\left(\bmod p^{n}\right) \quad \text { et } \quad v_{p}\left(\frac{\partial f}{\partial X_{j}}(x)\right)=k .
$$

Alors il existe un zéro $y$ de $\int$ dans $(p)^{m}$ qui est congru à $x$ modulo $p^{n-k}$.

\section{Démonstration :}

Ce théorème ainsi que les corollaires suivants sont démontré darns [10, pp. 28-30].

\section{Corollaire 1.36}

Soit $p$ impair, et $u$ une unité de $\Xi_{p}$; alors

$$
u \in U^{2}\left(-_{p}\right) \text { si et seulement si la congruence } u \equiv X^{2} \quad(\bmod p) \text { est résoluble. }
$$

De même si $p=2$, on a l'écuivalence:

$$
u \in U^{2}\left(\cdots_{2}\right) \text { si cu seulement si la congruence } u \equiv X^{2} \quad(\bmod 8) \text { est résoluble. }
$$

$$
\text { C'est-à-dire si et seulement si } u \equiv 1 \quad(\bmod 8) \text {. }
$$

\section{Corollaire 1.37}

Soit $A=\left(a_{i j}\right) \in G L_{n}\left(\mathbb{Z}_{p}\right)$ telle que $A=A^{t}$. Soient $f=\sum_{i, j=1}^{n} a_{i j} X_{i} X_{j} \in \mathbb{Z}_{p}\left[X_{1} \ldots, X_{n}\right]$ et $a \in \mathbb{Z}_{p}$.

Alors il existe $\left(\alpha_{1}, \ldots, \alpha_{n}\right) \in\left(E_{p}\right)^{n}$ primilif tel que $\int\left(\alpha_{1}, \ldots, \alpha_{n}\right)=a$ si et seulement si

a) $p$ impair : il existe $x_{1}, \ldots, x_{n} \in \cdots_{p}$ non tous dans $p_{\ldots}$, tels que $f\left(x_{1}, \ldots, x_{n}\right) \equiv a(\bmod p)$.

b) $p=2$ : il existe $x_{1}, \ldots, x_{n} \in \ldots_{2}$ non tous pairs tels que $f\left(x_{1}, \ldots, x_{n}\right) \equiv a \quad(\bmod 8)$.

Forts de ces résultats, nous allons étudier en détail les modules de $\mathscr{S}_{n}$ vus sur les anneaux p-adiques.

\section{Proposition 1.38}

Soit $(M, \beta)$ un $\mathbb{Z}_{p}$-module bilinéaire non dégénéré libre de rang $n$. Alors

$$
M \simeq<s_{1}>\boxplus \cdots \boxplus<s_{1}>\boxplus<\left(\begin{array}{ll}
a_{1} & c_{1} \\
c_{1} & b_{1}
\end{array}\right)>\boxplus \cdots \boxplus<\left(\begin{array}{ll}
a_{m} & c_{m} \\
c_{m} & b_{m n}
\end{array}\right)>
$$

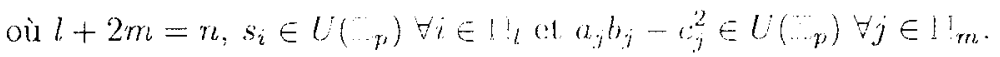

\section{Démonstration :}

Supposons qu'il existe $x_{1} \in M$ tel que $\beta\left(x_{1}, x_{1}\right) \in U\left(w_{p}\right) ; x_{1}$ est primitif, car si $x_{1}=p x_{1}^{\prime}$ alors $\beta\left(x_{1}, x_{1}\right)=p^{2} \beta\left(x_{1}^{\prime}, x_{1}^{\prime}\right) \notin U\left(z_{p}\right)$. De plus, $\left.\beta\right|_{\left\langle x_{1}\right\rangle}$ est non dégénérée, donc grâce à la proposition 1.5 et au corollaire 1.18 on a $\left.M \simeq\left\langle x_{1}\right\rangle \boxplus<x_{1}\right\rangle^{-}$. 
En continuant ainsi, on a

$$
M \simeq\left\langle x_{1}>\boxplus \cdots \boxplus<x_{l}>\boxplus M^{\prime} \quad \text { avec } \beta(x, x) \in p Z_{p} \forall x \in M^{\prime} .\right.
$$

Soit $z_{1} \in M^{\prime}, z_{1}$ primitif. P'uisque $\left.\beta\right|_{M}$, est non dégénérée, il existe $t_{1} \in M^{\prime}$ primitif tel que $\beta\left(z_{1}, t_{1}\right) \in$ $U\left(z_{p}\right)$. Nous savons que $\beta\left(z_{1}, z_{1}\right)$ at $\beta\left(t_{1}, t_{1}\right) \in \gamma_{p}$. Un rapide raisonnement de determinant nous permet de dire que $\left.\beta\right|_{\left\langle z_{1}, t_{1}\right\rangle}$ est non dégénérée.

De plus il est possible d'étendre $z_{1}, t_{1}$ en une ${ }_{-2}$-base de $M^{\prime}$, car soit $c=\frac{1}{p}\left(z_{1}+t_{1}\right)$, alors

donc $c \notin M$.

$$
\left|\beta\left(c, z_{1}\right)\right|=\left|\frac{1}{p} \beta\left(z_{1}, z_{1}\right)+\frac{1}{p} \beta\left(z_{1}, t_{1}\right)\right| \stackrel{(*)}{=}\left|\frac{1}{p} \beta\left(z_{1}, t_{1}\right)\right|=p>1
$$

L'égalité $(*)$ vient du fait que la valeur absolue $p$-adique est ultramétrique et que dans ce cas $|x+y|=$ $\max (|x|,|y|)$ si $|x| \neq|y|$.

Si $c=\frac{1}{p} z_{1}+t_{1}$ on voit de même que $\left|\beta\left(c, \iota_{1}\right)\right|=p$. En utilisant le théorème 1.17 et en faisant une brève récurrence, on conclut.

\section{Cas $p \neq 2$}

\section{Proposition 1.39}

Sous les mêmes hypothèse que la proposition précédente, avec $p$ impair, alors $(M, \beta)$ est diagonalisable.

\section{Démonstration :}

Grâce à la proposition 1.38 , il sullit de voir qu'une forme de dimension 2 et représentée par la matrice inversible $\left(\begin{array}{ll}a & c \\ c & b\end{array}\right)$, avec $a, b \in p_{p}, a \in \in U\left(\cdots_{p}\right)$, os diagonalisable.

Le vecteur $x=\left(\begin{array}{l}1 \\ 1\end{array}\right)$ représente $a+b+2$ : qui ost inversible si $p \neq 2$. $\left.\beta\right|_{<x>}$ étant donc non dégénéróe, la forme cst done diagonalisable.

\section{Lemme 1.40}

Soient $a, b, c \in \mathbb{E}_{p}$. Alors il existe $x$ et $y \in \mathbb{R}_{p}$ tels que $a x^{2}+b y^{2}=c$.

\section{Démonstration :}

Soit $A=\left\{a x^{2} \mid x \in \mathbb{F}_{p}\right\}$ et $B=\left\{c-b y^{2} \mid y \in \mathbb{F}_{p}\right\}$.

On a $\sharp A=\sharp B=\frac{p-1}{2}+1=\frac{p+1}{2}$. Ionc $A \cap B \neq 0$

\section{Proposition 1.41}

Soient $p \in \mathbb{P} \backslash\{2\}, a_{1}, \ldots, a_{n} \in U(\ldots$,$) a (M, \beta)$ un module bilinćaire libre de rang $n$ sur $\mathbb{Z}_{p}$, avec

$$
\beta=\left\langle a_{1}>\boxplus \cdots \boxplus<a_{n}\right\rangle .
$$

Alors $\beta$ est $Z_{p}$-équivalente à la forme

$$
<1>\boxplus \cdots \boxplus<1>\boxplus<a>
$$

où $a=\prod_{i=1}^{n} a_{i}$.

\section{Démonstration :}

Par le lemme précédent, nous savons quil cxiste $s_{1}$ ot $s_{2} \in \ldots_{p}$ tels que

$$
a_{1} s_{1}^{2}+a_{2} s_{2}^{2} \equiv 1 \quad(\bmod p)
$$

Sans limiter la généralité, on peut supposer que $s_{1}$ est inversible, et done que $\frac{1-a_{2} s_{2}}{a_{1}}$ est un carré modulo $p$. Vu le corollaire $1.36, i]$ existe done $\bar{s}_{1} \in U^{2}\left(\omega_{p}\right)$ tel que $a_{1}{\overline{s_{1}}}^{2}+a_{2} s_{2}^{2}=1$. Ceci démontre la proposition pour $n=2$; le cas $n$ quelconque se traite par une récurrence facile. 


\section{Corollaire 1.42}

Soit $p \in \mathbb{F}^{\prime} \backslash\{2\}$ et $(M, \beta) \in \mathscr{S}_{n}$. Alors

$$
\beta \stackrel{\stackrel{a}{\simeq}}{\simeq}<1>\boxplus \cdots \boxplus<1>
$$

\section{Démonstration :}

$\beta$ est diagonalisable vu la propositon 1.39, ot on conclut grâce à la proposition précédente, sachant que $\operatorname{det}(\beta)=1$.

\section{Cas $\mathrm{p}=2$}

\section{Remarque :}

Jusqu'ici, on voit qu'il n'est done pas nécessaire do se préoccuper des types; ce n'est que pour $p=2$ qu'il faudra distinguer le type $1 /$ et le type 1 .

\section{Modules de type $/ I$}

Soit $(M, \beta)$, un $\mathbb{Z}$-module bilinéaire de type $/ 1$. Par la proposition 1.38, et puisque $\beta(x, x) \in 2 \mathbb{Z}_{2} \forall x \in M_{2}$, on peut considérer que $\beta$ est une somme orthogonale de formes représentées par des matrices du type $\left(\begin{array}{ll}a & c \\ c & b\end{array}\right), a, b \in 2 \Xi_{2}$ el $c \in U\left(\sigma_{2}\right)$.

Nous allons voir que dans ce cas

$$
\left(\begin{array}{ll}
a & c \\
c & b
\end{array}\right) \stackrel{2}{\simeq} \begin{cases}\left(\begin{array}{ll}
0 & 1 \\
1 & 0
\end{array}\right) & \text { si }\left(\begin{array}{ll}
a & c \\
c & b
\end{array}\right) \text { représente } 0 \text { non trivialement } \\
\left(\begin{array}{ll}
2 & 1 \\
1 & 2
\end{array}\right) & \text { sinon. }\end{cases}
$$

Supposons donc qu'elle représente 0 non trivialement, c'est-à-dire, vu le corollaire 1.37, que l'équation

$$
a x^{2}+2 c x y+b y^{2} \equiv 0 \quad(\bmod 8)
$$

possède une solution $(x, y) \notin(2 \ldots 2)^{2} ;$ ce qui cst toujours le cas sauf si $a \equiv b \equiv 2 \bmod 4$. I] est clair que, dans ce cas, on a

$$
\left(\begin{array}{ll}
a & c \\
c & b
\end{array}\right) \rightleftharpoons\left(\begin{array}{ll}
0 & c^{\prime} \\
c^{\prime} & b^{\prime}
\end{array}\right)
$$

avec $b^{\prime} \in 2 \widetilde{Z}_{2}$ et $c^{\prime} \in U\left(\omega_{2}\right)$, car on peut supposer que 0 est représenté primitivement.

Finalement, la matrice $\left(\begin{array}{cc}\frac{-b^{\prime}}{2 f^{\prime}} & 1 \\ \frac{c^{\prime}}{c^{\prime}} & 0\end{array}\right)$ est la matrice de changement de base qui transforme $\left(\begin{array}{cc}0 & c^{\prime} \\ c^{\prime} & b^{\prime}\end{array}\right)$ en la matrice $\left(\begin{array}{ll}0 & 1 \\ 1 & 0\end{array}\right)$.

Si la forme ne représente pas 0 , c'est-à-dire si $a \equiv b \equiv 2 \bmod 4$, on a alors que l'équation

$$
a x^{2}+2\left(x y+b y^{2} \equiv 2 \quad(\bmod 8)\right.
$$

est résoluble avec $(x, y)=(1,1)$ ou $(1,-1)$ par exemple. Le corollaire 1.37 implique que notre matrice est $-z_{p}$-équivalente à $\left(\begin{array}{cc}2 & a^{\prime} \\ a^{\prime} & b^{\prime}\end{array}\right)$ avec $b^{\prime} \in 2 \ldots_{2}$ ot $a^{\prime} \in U\left(w_{2}\right)$.

Ce qui nous fait que $2 b^{\prime}-a^{2} \equiv 3 \operatorname{mond} 8$ car $b^{\prime} \equiv 2 \bmod 4$.

Il existe done $\alpha \in U\left(w_{2}\right)$ tel que $x^{2}\left(2 b^{\prime}-c^{2}\right)=3$.

En faisant un changement de base défini par la matrice $\left(\begin{array}{cc}1 & 0 \\ \frac{1-c a x}{2} & \alpha\end{array}\right)$, la matrice $\left(\begin{array}{cc}2 & c^{\prime} \\ c^{\prime} & b^{\prime}\end{array}\right)$ est $\mathbb{Z}_{p^{-}}$ équivalente à $\left(\begin{array}{ll}2 & 1 \\ 1 & 2\end{array}\right)$

Nous voilà donc en mesure de démontrer le 


\section{Théorème 1.43}

Si $(M, \beta)$ est un 2 -module bilinéaire de rang $2 r$ non dégénéré de type ( $/ /)$, alors :

\section{Démonstration :}

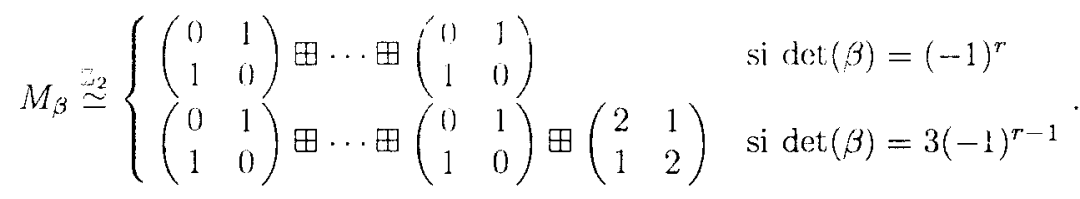

Vu ce qui précède, on a :

$$
\left(\begin{array}{ll}
2 & 1 \\
1 & 2
\end{array}\right) \stackrel{\mathscr{m}_{2}}{\simeq}\left(\begin{array}{ll}
-2 & -1 \\
-1 & -2
\end{array}\right)
$$

car $-2 \equiv 2 \bmod 4$. Donc il est évident que:

$$
\left(\begin{array}{llll}
2 & 1 & 0 & 0 \\
1 & 2 & 0 & 0 \\
0 & 0 & 2 & 1 \\
0 & 0 & 1 & 2
\end{array}\right) \stackrel{2}{\simeq}\left(\begin{array}{cccc}
2 & 1 & 0 & 0 \\
1 & 2 & 0 & 0 \\
0 & 0 & -2 & -1 \\
0 & 0 & -1 & -2
\end{array}\right)
$$

Mais de plus, nous avons l'égaticé matriciello suivante:

$$
\left(\begin{array}{cccc}
1 & 1 & 1 & 0 \\
1 & 1 & 0 & -1 \\
1 & 0 & 1 & -1 \\
0 & 1 & 1 & -1
\end{array}\right) \cdot\left(\begin{array}{llll}
0 & 1 & 0 & 0 \\
1 & 0 & 0 & 0 \\
0 & 0 & 0 & 1 \\
0 & 0 & 1 & 0
\end{array}\right) \cdot\left(\begin{array}{cccc}
1 & 1 & 1 & 0 \\
1 & 1 & 0 & 1 \\
1 & 0 & 1 & 1 \\
0 & -1 & -1 & -1
\end{array}\right)=\left(\begin{array}{cccc}
2 & 1 & 0 & 0 \\
1 & 2 & 0 & 0 \\
0 & 0 & -2 & -1 \\
0 & 0 & -1 & -2
\end{array}\right),
$$

on obtient donc que

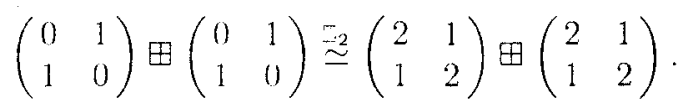

Enfin, la remarque (*) nous permet de terminer la démonstration.

\section{Corollaire 1.44}

Si $(M, \beta)$ est un module de $\mathscr{F}_{n}$ de type $(I /)$, alors $\left(M^{\prime}, \beta^{\prime}\right) \in \mathscr{F}_{n}$ est de même genre que $(M, \beta)$ si et seulement si $\left(M^{\prime}, \beta^{\prime}\right)$ est de type $(I I)$. Autrement dit, $\mathscr{C}_{n}$ représente un et un seul genre.

\section{Démonstration :}

Si $\left(M^{\prime}, \beta^{\prime}\right)$ est de type $(I I)$, alors on a vu au corollaire 1.42 que

$$
\beta_{p}^{\prime} \stackrel{\stackrel{*}{\sim}}{\sim}<1>\boxplus \cdots \boxplus<1>
$$

si $p \neq 2$. Et, si $p=2$, par le théoreme précédent on a :

$$
\beta_{2}^{\prime} \stackrel{p}{\simeq}\left(\begin{array}{ll}
0 & 1 \\
1 & 0
\end{array}\right) \boxplus \cdots \boxplus\left(\begin{array}{ll}
0 & 1 \\
1 & 0
\end{array}\right)
$$

$\operatorname{car} \operatorname{det}\left(\beta^{\prime}\right)=1$.

La réciproque est évidente.

\section{Remarque :}

Il faut tout de même signaler que les formes bilinéaires de $\mathscr{P}_{n}$ de type $(I I)$ sont plutôt rares dans les petites dimensions; le corollaire précédent montre déjà que $n \equiv 0 \bmod 4$, mais on peut montrer qu'en fait $n \equiv 0 \operatorname{rrod} 8$; ceci est démontré dans [10, p. 92].

La forme représentée par la matrice.

$$
\Gamma_{8}=\left(\begin{array}{cccccccc}
2 & 0 & -1 & 0 & 0 & 0 & 0 & 0 \\
0 & 2 & 0 & -1 & 0 & 0 & 0 & 0 \\
-1 & 0 & 2 & -1 & 0 & 0 & 0 & 0 \\
0 & -1 & -1 & 2 & -1 & 0 & 0 & 0 \\
0 & 0 & 0 & -1 & 2 & -1 & 0 & 0 \\
0 & 0 & 0 & 0 & -1 & 2 & -1 & 0 \\
0 & 0 & 0 & 0 & 0 & -1 & 2 & -1 \\
0 & 0 & 0 & 0 & 0 & 0 & -1 & 2
\end{array}\right)
$$

est le plus simple exemple d'une telle forme. 


\section{Modules de type I}

\section{Lemme 1.45}

Si $(M, \beta) \in \mathscr{F}_{n}$ est de typo $(I)$ alors $\beta$ est $\tau_{2}$-équivalente à une forme diagonale.

\section{Démonstration :}

Par définition, il existe $e_{1}$ tel que $\beta\left(e_{1}, c_{1}\right)=u_{1} \in U\left(\mathbb{Z}_{2}\right)$. Donc $\beta \simeq<u_{1}>⿴ 囗 \beta^{\prime}$ avec $\beta^{\prime}$ non dégénérée.

Si $\beta^{\prime}$ représente une unité de $\mathbb{Z}_{2}$, on continue; sinon, on considère $\left(e_{2}, \ldots, e_{n}\right)$ une base de $\left\langle e_{1}\right\rangle^{\perp}$.

Soit $e_{1}^{\prime}=e_{1}+e_{2}$. Clairement $\beta\left(e_{1}^{\prime}, e_{1}^{\prime}\right)=u_{1}^{\prime}$ est impair, car $\beta\left(e_{2}, e_{2}\right)$ est pair; donc $\left.\beta \simeq<u_{1}^{\prime}\right\rangle \boxplus \beta^{\prime \prime}$.

Montrons que $\beta^{\prime \prime}$ est de type $(I)$ :

$\beta^{\prime}$ étant non dégénérée, il existe $h \in\left\langle e_{1}\right\rangle-$ tel que $\beta\left(h, e_{2}\right)=1$. Soit $t=e_{1}-u_{1} h$; on a

$$
\beta(t, h)=\beta\left(c_{1}, c_{1}\right)+u_{1}^{2} \beta(h, h)=\text { impair + pair } \in U\left(\mathbb{Z}_{2}\right)
$$

puisque on a supposé $\beta(h, h)$ pair. De plus

$$
\beta\left(t, c_{1}^{\prime}\right)=\beta\left(c_{1}-u_{1} h, e_{1}+c_{2}\right)=u_{1}-u_{1} \beta\left(h, e_{2}\right)=0 .
$$

En résumé, $t \in\left\langle e_{1}^{\prime}\right\rangle$ et $t$ représente uno unité. Done $\beta^{\prime \prime}$ est de type $(I)$.

On peut donc conclure par récurrence.

Remarquons que tous les éléments de cot te diagonale sont impairs, eu le produit de ces éléments est 1.

\section{Définition 1.46}

Soit $p$ un nombre premier, $(M, \beta)$ unc forme bilinéaire sur $\hat{i}_{p}$. On suppose que $\beta$ est $\mathbb{Z}_{p}$-équivalente à une forme diagonale

$$
<a_{1}>\boxplus \cdots \boxplus<a_{n}>.
$$

On définit alors

$$
c_{p}(\beta)=\prod_{i<j}\left(a_{i}, a_{j}\right)
$$

où $\left(a_{i}, a_{j}\right)$ est le symbole de Hilbcrt. Io nombre $c_{p}(\beta)$ est appelé l'invariant de Hasse-Minkowski.

\section{Lemme 1.47}

$c_{p}(\beta)$ est indépendant de la diagonalisation choisic. En particulier, si deux formes diagonales sont $\mathbb{Z}_{p^{-}}$ équivalentes, alors elles ont le mëme invariant de Ilasse-Minkowski.

\section{Démonstration :}

Ce résultat est dérnontré dans [3, p.57].

\section{Théorème 1.48}

Si $(M, \beta) \in \mathscr{P}_{n}$ est de type $(I)$, alors $\beta$ cst $\sim_{2}$-équivalente à la forme $\langle 1>\boxplus \cdots \boxplus<1>$.

\section{Démonstration :}

On peut supposer grâce au lemme 1.45 que notre forme est diagonale.

Soient $c \in \mathbb{Z}_{2}$ et $a_{1}, \ldots, a_{4} \in U\left(Z_{2}\right)$. Alors il existe une solution à l'équation

$$
a_{1} x_{1}^{2}+\cdots+a_{1} x_{4}^{2} \equiv c \quad(\bmod 8)
$$

En effet, considérons

$A=\left\{y \mid y=a_{1} x_{1}^{2}+a_{2} x_{2}^{2}, x_{1}, x_{2} \in \cdots / s\right\}$ ot $l 3=\left\{y \mid y=c-a_{3} x_{3}^{2}-a_{4} x_{4}^{2}, x_{1}, x_{2} \in=/ 8=\right\}$. 
Une vérification directe nous permet do voir que le cardinal de chacun de ces ensembles est au moins 5 .

On trouve done que $A \cap B \neq \emptyset$.

Si on applique ce résultat pour $c=1$ ainsi que le corollaire 1.37 , il est clair que

$$
\beta \simeq<1>\boxplus \cdots \boxplus<1>\boxplus<a_{1}>\boxplus<a_{2}>\boxplus<a_{3}>
$$

avec $\prod a_{i} \equiv 1 \bmod 8$.

S'il y a deux " 5 " ou un " 1 " parmi los $a_{i}$, la forme $\left.\left\langle a_{1}\right\rangle \boxplus<a_{2}\right\rangle \boxplus\left\langle a_{3}\right\rangle$ représente 1 , donc nous pouvons poursuivre le procédé; par contre si les $a_{i}$ ne sont pas de cette forme, il ne nous reste que le cas $a_{1}=3, a_{2}=5, a_{1}=7$, (les autres ne sont pas de détcrminant 1 ), mais 1 est représenté par $x_{1}=2, x_{2}=1$ et $x_{3}=0$.

Nous avons done avancé d'un cran, et maintenant.

$$
\beta \simeq<1>\boxplus \cdots \boxplus<1>\boxplus<a_{1}>\boxplus<a_{2}>
$$

avec $a_{1} \cdot a_{2} \equiv 1 \bmod 8$.

Il s'ensuit que $a_{1}=a_{2}=a$. Si $a=1$ ou 5 , le problème est réglé.

Et puisque $<3>\boxplus<3>\simeq<7>\boxplus<7>$ (la matrice de changement de base est $\left(\begin{array}{cc}2 \alpha & \alpha \\ \alpha & -2 \alpha\end{array}\right)$ avec $\alpha \in U\left(\mathbb{Z}_{2}\right)$ tel que $15 \alpha^{2}=7$ ) il ne nous reste que le cas $a=3$.

Pour le traiter, nous avons besoin de linvariant de Hasse-Minkowski avec toute son armada.

Tout d'abord, on a que $c_{p}(\beta)=1 \forall p \in\left\{\{2\}\right.$ car pour de tels $p$, on a $\beta \stackrel{\mathbb{Z}_{p}}{\simeq}<1>\boxplus \ldots \boxplus<1>$; cela découle du corollaire 1.42 ainsi que d'un théorme conmu sur les formes définies positives sur $\mathbb{R}$.

Vu la formule du produit de Hilbert et le lemme précédent, on en déduit que $c_{2}(\beta)=1$. Or calculons $c_{2}\left(\beta^{\prime}\right)$ ò̀

$$
\beta^{\prime}=\langle 1>\boxplus \cdots \boxplus<1>\boxplus<3>\boxplus<3>：
$$

vu le théorème 1.31 , on a

$$
(3,3)_{2}=(-1)^{\frac{(3-1)(3-1)}{4}}=-1 ;
$$

de plus, $(1,3)_{2}=(1,1)_{2}=1$. 1)'ò̀ or a que $c_{2}\left(\beta^{\prime}\right)=-1$; ce qui lait que $\beta \not \beta^{\prime}$ en vertu du lemme précédent.

Donc $\beta \simeq<1>\boxplus \cdots \boxplus<1>\boxplus<a>$; mais lì, puisque le déterminant vaut, 1 , on a que $a=1$.

\section{Corollaire $\mathbf{1 . 4 9}$}

La relation d'équivalence "être dans lo même genre que" définie sur $\mathscr{S}_{n}$ ne comporte que deux classes d'équivalences: les formes de lype $(I I)$ et les formes de types $(I)$.

\section{Démonstration :}

C'est un corollaire immédiat du théorène précédent et du corollaire 1.44.

\section{G. Enoncé du problèmo}

Nous voilà enfin en mesure de poser clairement le problème.

On se fixe $(M, \beta)$ un -module bilinćaire de $\mathscr{P}_{n}$, et on pose $V=M \otimes$ et $\mathscr{F}_{n}$ l'ensemble des classes à isomorphisme près des éléments de $\mathscr{F}_{n}$ qui sont darıs le même genre que $(M, \beta)$. Le corollaire 1.49 montre que $\mathscr{Z}_{n}=\mathscr{C}_{n}$ ou $\mathscr{H}_{n}$.

l.e théorème de Hasse-Minkowski nous permot do dire que tous les représentants de ces classes d'équivalences peuvent etre vus comme des réscaux biliníaires de $V$.

Soient $M_{1}, \ldots, M_{k}$ des représentants de chaque dasse de $\mathscr{F}_{n}$ 
et $O\left(M_{i}\right)$ le groupe orthogonal do $M_{i} \forall i \in !_{k} . O\left(M_{i}\right)$ est un groupe fini car isomorphe à un sous-groupe discret du groupe orthogonal de $M_{i} \otimes$ qui est compact.

Nous nous proposons de calculer

$$
\mathscr{M}_{n}=\sum_{i=1}^{k} \frac{1}{\left|O\left(M_{i}\right)\right|}
$$

Ce nombre rationnel est appelé masse de $\mathscr{Z}_{n}$.

Il existe une formule pour calculer $\mathscr{M}_{n}$; c'cst à la démontration et au calcul de cette formule que seront consacrés les chapitres suivants. Son nom est la formule de Minkowski-Siegel.

Remarquons que si nous prenons un module $(M, q)$ où $q$ est une forme quadratique de la forme $\beta(x, x)$ avec $\beta \in \mathscr{S}_{n}$ et que nous faisons le même travail, nous obtenons les mêmes classes d'équivalences et les mêmes genres que précédemment. De plus, la formule de masse reste la même car les anneaux $p$-adiques ainsi que $\exists$ sont des anneaux integres. 


\section{CHAPITRE 2}

\section{Mesures, masses et formule de Minkowski-Siegel.}

Nous allons établir dans ce chapitre la formule de Minkowski-Siegel. Pour cela, il faudra faire un peu de théorie de la mesure sur les groupes orthogonaux.

A. Structure congruenticlle et mesure de Haar.

\section{Définitions 2.1}

Soit $G$ un groupe et $\mathscr{Z}$ une famille de sous-groupes satisfaisant les propriétés suivantes :

$\left(C_{1}\right)$ Si $K_{1}, K_{2} \in \mathscr{G}$, alors il existe $K_{3} \in \mathscr{G}$ tel que $K_{3} \subset K_{1} \cap K_{2}$.

$\left(C_{2}\right)$ Si $K_{1}$ et $K_{2} \in g$ sont tels que $K_{1} \subset K_{2}$, alors l'indice $\left[K_{2}: K_{1}\right]$ est fini.

$\left(C_{3}\right)$ Si $K \in \mathscr{g}$ et $u \in G$, alors $u \cdot K \cdot u^{-1} \in \mathscr{g}$.

On dit alors que $\mathscr{G}$ munit $G$ d'une structure congruentielle, et les éléments de $\mathscr{G}$ sont appelés sous-groupes de congruence principaux.

On définit aussi

$$
\mathscr{E}=\left\{\bigcup_{i=1}^{n} x_{i} K_{i} \mid n \in I I, x_{1}, \ldots, x_{n} \in G \text { et } K_{1}, \ldots, K_{n} \in \mathscr{Z}\right\}
$$

Les éléments de $\mathscr{E}$ sont appelés ensembles de congruence.

\section{Proposition 2.2}

A) $\mathscr{E}$ est stable par intersection ol réunion finics.

B) Si $E_{1}, \ldots, E_{n} \in \mathscr{E}$, alors il existc: $K \in \mathbb{Z}$ icl que pour tout $i \in I I_{n}, E_{i}$ soit réunion disjointe finie de classes à gauche modulo $K$.

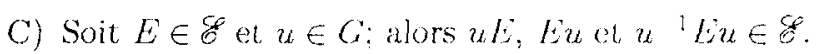

\section{Démonstration :}

Soient $E=\bigcup_{i} x_{i} K_{i}$ et $E^{\prime}=\bigcup_{j} y_{j} L_{, j} \in \mathscr{E}$. Il est clair que $E \cup E^{\prime} \in \mathscr{E}$. De plus, $E \cap E^{\prime}=\bigcup_{i, j} x_{i} K_{i} \cap y_{j} L_{j}$. Or, pour tous sous-groupes $H$ et $H^{\prime}$ de $G$, si $x H \cap y H^{\prime}$ est non vide, on a l'égalité suivante:

$$
x H \cap y H^{\prime}=z\left(H \cap H^{\prime}\right) \text { pour tout } z \in x H \cap y H^{\prime} .
$$

Ce résultat s'obtient gràce à une vérification évidente. Donc, on a que $E \cap E^{\prime}=\bigcup z_{i j}\left(K_{i} \cap L_{j}\right)$, en supposant que $K_{i} \cap L_{j}$ est non vide pour tous $i, j$. Mais, pour tout sous-groupe de $\stackrel{i, j}{G}$ contenant un $K$ dans $\mathscr{Z}$ et tel que $[H: K]<\infty$, on a que $H \in \mathscr{E}$; ceci nous permet de dire que $K_{i} \cap L_{j} \in \mathscr{E}$ pour tous $i, j$, grâce aux axiomes $\left(C_{1}\right)$ et $\left(C_{2}\right)$.

Soient maintenant $E_{1}, \ldots, E_{n} \in \mathscr{E}$. Par définition, on a pour tout $i$ :

$$
E_{i}=\bigcup_{j} x_{j} K_{i j} \quad \text { avec } K_{i j} \in \mathscr{g} \forall i, j
$$

L'axiome $\left(C_{1}\right)$ nous permet de prendre un sous-greupe de congruence principal $K$ contenu dans $\bigcap_{i j} K_{i j}$ et l'axiome $\left(C_{2}\right)$ nous permet de dire que $K_{i j}$ est une réunion disjointe finie de classes à gauche modulo $K$. Le fait que pour tout sous-groupe $/ / \mathrm{d}: G$ on ait $x H \cap y H \neq \emptyset \Longrightarrow x H=y H$ nous permet de terminer la démonstration de $B$ ).

D'autre part, si $E$ est dans $\mathscr{E}$, alors $u E$ et $u^{-1} E u \in \mathscr{E}$, et comme $E u=u\left(u^{-1} E u\right)$, on conclut. 


\section{Définition 2.3}

Soit $(G, \mathscr{g})$ un groupe muni d'une structure congruentielle. Une mesure de Haar est une application $\mu$ de $\mathscr{E}$ dans $F_{+}$telle que :

$$
\begin{aligned}
& \text { (I) } \mu \neq 0 \\
& \text { (II) si } E_{1}, E_{2} \in \mathscr{E} \text { sont tels que } E_{1} \cap E_{2}=\emptyset, \text { alors } \mu\left(E_{1} \cup E_{2}\right)=\mu\left(E_{1}\right)+\mu\left(E_{2}\right) \\
& (I I I) \mu(u E)=\mu(E) \text { pour tout } u \in G \text { et } E \in \mathscr{E} .
\end{aligned}
$$

\section{Théorème 2.4}

Si $G$ est un groupe muni d'une structure congruentielle $\mathscr{G}$, alors il existe une mesure de Haar, et si $\mu_{1}$ et $\mu_{2}$ sont deux mesures de Haar, alors il existe une constante $c>0$ telle que $\mu_{2}=c \mu_{1}$.

\section{Démonstration :}

a) Fixons $E_{0} \in \mathscr{E}$ tel que $E_{0} \neq \emptyset$. Soil $E \in \mathscr{E}$ quelconque; par la proposition 2.2 , on peut choisir $K \in \mathscr{Z}$ tel que $E_{0}=\bigsqcup_{i=1}^{r} x_{i} K$ el $E=\bigsqcup_{j=1}^{s} y_{j} K$. Posons alors $\mu(E)=\frac{s}{r}$. Supposons que $E_{0}=\bigsqcup_{i=1}^{r^{\prime}} x_{i}^{\prime} K^{\prime}$ et que $E=\bigsqcup_{j=1}^{s^{\prime}} y_{j}^{\prime} K^{\prime}$ et voyons que $\frac{s}{r}=\frac{s^{\prime}}{r^{\prime}}$

$\operatorname{Par}\left(C_{1}\right)$, nous savons qu'il existe $L \in \mathscr{Y}^{\prime}$ tel que $L \subset K^{\prime} \cap K$, puis par $\left(C_{2}\right)$, il existe $m$ et $m^{\prime} \in \mathbb{N}$ tel que

$$
K=\bigsqcup_{t=1}^{m} z_{t} L \quad \text { et } \quad K^{\prime}=\bigsqcup_{k=1}^{m^{\prime}} z_{k}^{\prime} L
$$

done

$$
E_{i}=\bigsqcup_{i}^{r} \bigsqcup_{l=1}^{m} x_{i} z_{i} L=\bigsqcup_{i=1}^{r^{\prime}} \bigsqcup_{k=1}^{m^{\prime}} x_{i}^{\prime} z_{i}^{\prime} L
$$

On a alors que $r m=r^{\prime} m^{\prime}$. Par un même raisonnement, on obtient que $s m=s^{\prime} m^{\prime}$, d'où $\frac{s}{r}=\frac{s^{\prime}}{r^{\prime}}$. Donc $\mu$ est bien défnie.

Il est clair que $\mu \neq 0$ et que $\mu(u E)=\mu(E)$ pour tout $u \in G$ et $E \in \mathscr{E}$. Soient maintenant $E$ et $E^{\prime}$ dans $\mathscr{E} ;$ vu la proposition 2.2 , il existe $K$ tel que

$$
E_{0}=\bigsqcup_{i=1}^{r} x_{i} K, E=\bigsqcup_{j=1}^{s} y_{j} K \text { et } E^{\prime}=\bigsqcup_{l=1}^{s^{\prime}} y_{l}^{\prime} K
$$

Mais, puisque $E$ et $E^{\prime}$ sont disjoints, on a que $y_{j} K \neq y_{l}^{\prime} K \forall j, l$. Donc

$$
E \sqcup E^{\prime}=\bigsqcup_{i=1}^{s+s^{\prime}} z_{i} K
$$

où $z_{i}=y_{i} \forall i \in I_{s}$ et $z_{s+i}=y_{i}^{\prime} \forall i \in I_{s^{\prime}}$. Done finalement,

$$
\mu\left(E \cup E^{\prime}\right)=\frac{s+s^{\prime}}{r}=\frac{s}{r}+\frac{s^{\prime}}{r}=\mu(E)+\mu\left(E^{\prime}\right) .
$$

b) Soient $\mu_{1}$ et $\mu_{2}$, deux mesures de Jlaar sur $(C, q)$. lixons-nous $E_{0} \in \mathscr{E}$ tel que $\mu_{1}\left(E_{0}\right) \neq 0$. Il existe $c \geq 0$ tel que $\mu_{1}\left(E_{0}\right)=c_{\mu_{2}}\left(E_{i}\right)$. Sorit $E \in E$, il cxiste $K \in G^{g}$ tel que $E_{0}=\bigsqcup_{i=1}^{r} x_{i} K$ et $E=\bigsqcup_{j=1}^{s} y_{j} K$. On a :

$$
\mu_{1}(E)=s \mu_{1}(K)=\frac{s}{r} \mu_{1}\left(E_{0}\right)=\frac{s}{r} c \mu_{2}\left(E_{0}\right)=c s \mu_{2}(K)=c \mu_{2}(E) .
$$

Et, nous voyons que $c>0$, puisque $\mu_{1}$ et $\mu_{2}$ sont non nulles. 


\section{Définition 2.5}

Soient $G$ un groupe muni d'une structure congruentielle et $\mu$ une mesure de Haar. Définissons

$$
\begin{aligned}
\mu_{u}: \mathscr{E} & \longrightarrow \mathbb{R}+ \\
E & \longmapsto \mu(E u) .
\end{aligned}
$$

C'est clairement une mesure de Haar. Donc, par le lemme précédent, il existe $c(u)$ tel que $\mu_{u}=c(u) \mu$. On obtient alors une application $a$ de $C$ dans 10 , attachée à la structure congruentielle, et telle que $c(u v)=c(u) c(v) \forall u, v \in G$. Couc application s'appolle la fonction modulaire do $(G, \mathscr{G})$, et $(G, \mathscr{G})$ est dit unimodulaire si c: est identiquernent 1.

\section{B. Groupe orthogonal et structure congruentielle.}

Dans ce paragraphe, nous allons montrer qu’il est possible de définir une structure congruentielle sur le groupe orthogonal d'un espace vectoriel bilinéaire ou quadratique donné.

\section{Définitions 2.6}

Fixons-nous, pour le reste de ce paragraphe, $A$ un arneau principal à quotient fini et de caractéristique différente de $2, K$ son corps des fractions et $V$ un espace vectoriel de dimension $n$ sur $K$. Munissons $V$ d'une forme bilinéaire non dégćnćrée $\beta$. On notera $q$, la forme quadratique définie par $q(x)=\beta(x, x)$. Au lieu de partir d'une lorme bilinéaire, prenons q une forme quadratique non dégénérée sur $V$. Posons $\beta$ définie par $\beta(x, y)=q(x+y)-q(x)-q(y)$. Dans les deux cas, puisque $A$ est intègre et de caractéristique différente de 2 , on a $O_{q}(V)=O_{\beta}(V)$. Nous noterons ce groupe $O(V)$.

Puisque par la suite, on considérera des espaces quadratiques et des espaces bilinéaires, mais que cela n'influe en ricn les raisonnements qui vont suivre, on dira que $V$ est un espace bilinéaire ou quadratique. Soient $N \subset M$ deux A-róscaux de $V ;$ on dérimit

$$
O(V, M / N)=\{u \in O(V) \mid u(M)=M, u(N)=N \text { ct } u(x) \equiv x \bmod N \forall x \in M\} .
$$

et

$$
O(V, M)=\{u \in O(V) \mid u(M)=M\} .
$$

Ces ensembles sont clairement des sous-groupes de $O(V)$. Si $M$ est un réseau quadratique ou bilinéaire, $O(V, M)$ sera noté $O(M)$.

Soit $g$ l'ensemble des sous-groupes de $O(V)$ de la forme $O(V, M / a M)$, où $a \in A$ et $M$ est un $A$-réseau quelconque de $V$. Nous allons montrer que $\mathscr{g}$ muit $O(V)$ d'une structure congruentielle.

\section{Lernme 2.7}

Soient $M_{1}$ et $M_{2}$, deux $A$-róscaux. Il existe $a$ et b dans $A$ tels que $a M_{1} \subset M_{2}$ et $r M_{2} \subset M_{1}$.

\section{Démonstration :}

On sait qu'il existe $\left(c_{1}, \ldots, c_{n}\right)$ et $\left(f_{1}, \ldots, f_{n}\right)$, deux $K$-bases de $V$ tels que

$$
\begin{aligned}
& M_{1}=A e_{1} \odot \cdots \oplus A e_{n} \\
& M_{2}=A d_{1} \odot \cdots \oplus A f_{n} .
\end{aligned}
$$

Soient $a_{i j} \in K$ tels que $e_{i}=\sum_{j}^{n} a_{i j} f_{j}$ pour tout $i \in !_{n}$, et a un dénominateur commun des $a_{i j}$. Nous avons alors

$$
a e_{i}=\sum_{i \cdots 1}^{n} a_{i, j}^{\prime} f_{j} \text { avec; } a_{i j}^{\prime} \in A \forall i, j \in I_{n}
$$

c'est à dire $a M_{1} \subset M_{2}$. 


\section{Remarque :}

Si $A=z$, alors on peut supposer que $a$ et $b$ sont des puissances de $p$.

\section{Lemme 2.8}

Soient $M$ un $A$-réseau et $N$ un sous- $A$-module de $M$; alors $N$ est un $A$-réseau, si et seulement s'il existe $a$ et $b$ dans $K$ tels que $a N \subset M \subset b N$.

\section{Dérnonstration :}

S'il existe $a, b \in K$ tels que $a N \subset M \subset b N$, alors $N$ est isomorphe à un sous-module de $M$; il est donc un module de génération finie, car la multiplication par a ou par $b$ est un isomorphisme $A$-linéaire de $N$ dans $a N$ ou $b N$. De plus, on a $K N \subset K M \subset K N$, ce qui nous donne $K N=K M=V$; donc $N$ est un $A$-réseau de $V$.

La réciproque est évidente grâce aux lemmes précédents.

\section{Lemme 2.9}

Soient $M$ et $N$, deux A-réseaux de $V$, alors $M+N$ et $M \cap N$ sont aussi des A-róseaux.

\section{Démonstration :}

Le lemme 2.7 nous dit qu'il existe $a$ at $b \in A$ tels que $a M \subset N$ ct $b N \subset M$. Or, nous avons immédiatement les inclusions suivantes:

$$
b(M+N) \subset b M+b N \subset M \subset M+N
$$

et

$$
M \cap N \subset M \subset \frac{1}{a} M \cap \frac{1}{a} N=\frac{1}{a}(M \cap N) .
$$

Ce qui démontre le lemme.

\section{Proposition 2.10}

Soient $O(V, M / b M)$ at $O(V, N / a N) \in Z$. les lemmes précédents nous donnent l'existence d'un $c$ tel que $c(M+N) \subset a N \cap b M$. Alors on a:

$$
O(V, M+N / c(M+N)) \subseteq O(V, M / b M) \cap O(V, N / a N) .
$$

\section{Démonstration :}

Soient $x \in M$ et $u \in O(V, M+N / c(M+N))$. En particulier, $x \in M+N$, donc $u(x)-x \in c(M+N) \subset$ $b M \subset M$. Ce qui nous donne que $u(x) \in M$ ot $u(x)-x \in b M$.

Par un même raisonnoment, si $y \in N$, nous obtcnons que $u(y) \in N$ et $u(y)-y \in a N$. Nous avons que $u(M) \subset M$; or, $O(V, M+N / c(M+N))$ est un groupe; alors, en faisant le même raisonnement pour $u^{-1}$, il vient que $u^{-1}(M) \subset M$, donc $M \in u(M)$.

\section{Proposition 2.11}

Soient $O(V, M / a M) \subset O(V, N / b N) \in \mathscr{F}$. Alors

$$
[O(V, N / b N): O(V, M / a M)]<\infty .
$$

\section{Démonstration :}

On sait qu'il existe $\ell$ a $r \in A$ tels que $\ell M \subset N$ et $r N \subset \ell a b M$. Une rapide vérification nous permet de montrer les inclusions suivantes:

$$
O(V, N / r N) \subset O(1, N / h a b, M) \subset O(V, M / a M) \subset O(V, N / b N)
$$


Il suffit donc de démontrer que $[O(V, N / r N): O(N / b N)]$ est fini.

Nous avons déjà que $r N \subset b N \subset N$. Soit maintenant

$$
\begin{aligned}
\varphi: O(V, N / b N) & \longrightarrow \mathscr{P}(N / r N) \\
u & \longrightarrow \varphi(u): N / r N \longrightarrow N / r N \\
x+r N & \longrightarrow u(x)+r N
\end{aligned}
$$

où $\mathscr{L}(N / r N)$ est le groupc des automorphismes de $N / r N$, vu comme $A / r A$-module. On a supposé que $A$ était à quotient fini; donc $\mathscr{L}(N / r N)$ est fini. Notre application $\varphi$ est bien définie, car tout élément $u$ de $O(V, N / b N)$ est tel que $u(N)=N$, donc $u(r N)=r N$. De plus, $\varphi$ est clairement un homomorphisme de groupe.

Etudions maintenant le noyau de cet homomorphisme:

Dire que $\varphi(u)=\varphi(v)$ pour $u, u \in O(V, N / h N)$ est equivalent à dire que $u^{-{ }^{1}} v(x) \equiv x$ mod $r N$ pour tout $x \in N$ et, comme $u^{-1} v(N)=N$, cola veut dire que $u^{-1} v \in O(V, N / r N)$.

En résumé, on a que $O(V, N / b N) / O(V, N / r N)$ est isomorphe à un sous-groupe de $\mathscr{L}(N / r N)$ qui est fini. Ce qui démontre la proposition.

\section{Proposition 2.12}

Soient $O(V, M / a M) \in \mathscr{I}$ et $u \in O(V)$. Alors

$$
u O(V, M / a M) u^{1}=O(V, u(M) / a u(M))
$$

\section{Démonstration :}

C'est immédiat.

\section{Théorème 2.13}

L'ensemble $\mathscr{Z}$ des sous-groupes de $O(V)$ de la forme $O(V, M / a M)$, où $a \in A$ et $M$ est un $A$-réseau quelconque de $V$, munit $O(V)$ d'une structure congruentielle. De plus, $(G, g)$ est unimodulaire. Finalement, si $M_{1}$ est un sous-A-réscau de $M_{2}$, alors $O\left(V, M_{2} / M_{1}\right)$ cst un ensomble de congruence.

\section{Démonstration :}

La première partie de ce thécrime st ure conséquence directe des propositions 2.10, 2.11 et 2.12 .

On a $(G, \mathscr{G})$ est unimodulaire, car $O(V)$ cst cngendré par les symétries orthogonales relativement aux hyperplans de $V$. Ce fait est démontró dans [3, lemrne 4.3, p. 20]. Puisque ces applications sont d'ordre fini dans $O(V)$, on en déduit que c est identiquement 1 , car dans $\mathbb{F}_{+}$, il n'y a pas de racine de l'unité autre que 1 lui-même.

Pour la dernière partie de ce théoreme, on sait qu'il existe $a \in A$ tel que $a M_{2} \subset M_{1}$. De plus, on a que $O\left(V, M_{2} / a M_{2}\right) \subset O\left(V, M_{2} / M_{1}\right)$. Si on définit l'application $\varphi$ de $O\left(V, M_{2} / M_{1}\right)$ dans $\mathscr{L}\left(M_{2} / a M_{2}\right)$ comme dans le lemme précédent, on voit que le noyau de cette application est $O\left(V, M_{2} / a M_{2}\right)$. Puisque $\mathscr{L}\left(M_{2} / a M_{2}\right)$ est fini, on en déduit que $O\left(V, M_{2} / M_{1}\right)$ est une réunion disjointe finie de $u_{i} O\left(V, M_{2} / a M_{2}\right)$, où $u_{i} \in O(V)$.

\section{Groupe orthogonal adélique et structure congruentielle.}

Fixons-nous, pour ce paragraphe, un -espace vectoriel $V$ muni d'une forme bilinéaire ou quadratique non dégénérée. Nous allons montrer quiil est possible de munir $\widetilde{O}(V)$ d'une strueture congruentielle, où $\widetilde{O}(V)$ est le groupe orthogonal adélique que nous délinirons tout à l'heure. 


\section{Lemme 2.14}

Soient $M$ un $\mathbb{Z}$-réseau de $V$, $\left(E_{p}\right)_{p \in \mathbb{T}}$ formées pour chaque $p$ d'un ${ }_{p}$-réseau de $V_{p}$, telles que $E_{p}=M_{p}$ pour tous les $p$ sauf pour un nombre fini (on dira par lia suite "pour presque tout $p$ "). Alors l'application

$$
\int: N \longmapsto\left(N_{p}\right)_{p \in \mathbb{F}}
$$

forme une bijection entre $\mathscr{R}$ et $\mathscr{C}_{M}$, avec

$$
f^{-1}:\left(N_{p}\right)_{p \in \mathbb{E}} \longmapsto \bigcap_{p \in \mathbb{P}^{*}}\left(N_{p} \cap V\right)
$$

\section{Démonstration :}

Montrons déjà que notre application f a bien son image dans $\mathscr{H}_{M}$ :

Soit $N \in \mathscr{R}$; nous savons qu'il existe a a $a^{\prime} \in \because$ tels que, $a N \subset M \subset a^{\prime} N$. Or, $a$ et $a^{\prime}$ sont inversibles dans $\ldots$ pour presque toul $p$. Donc

$$
a N_{p}=a^{\prime} N_{p}=N_{p}=M_{p}
$$

pour presque tout $p$, ct done $\left(N_{p}\right)_{W} \in \mathscr{Q}_{M}$.

Montrons maintenant que $f^{1}$ of est lidentité de

soient $N \in \mathscr{C}$ et $N_{p}=N \otimes$, . Il faut voir que $N=\bigcap_{p \in \mathbb{E}}\left(N_{p} \cap V\right)$. Le fait que $N \subset \bigcap_{p \in \mathbb{P}}\left(N_{p} \cap V\right)$ est évident. Soit $x \in \bigcap_{p \in \Theta}\left(N_{p}, \cap V\right)$; puisque $x \in V$, on peut écrire $x=\frac{y}{m}$, avec $y \in N$ et $m>0$ minimal. Mais $x \in N_{p}$, done $p$ ne divise pas $m$, quel que soit $p \in \mathbb{E}$, ce qui veut dire que $n,=1$ et donc $x=y$.

Il reste à voir que $\int \circ \int^{1}$ est l'identité do $\mathscr{Z}_{M}$ :

soit $\left(E_{p}\right)_{p \in \mathbb{P}} \in \mathscr{L} \mathscr{L}_{M} ;$ posons $N=\bigcap_{p \in \mathbb{E}}\left(E_{p} \cap V\right)$. Nous allons montrer dans un premier temps que $N$ est un $\mathbb{Z}$-réseau de $V$, cnsuite nous montrerons que $N_{p}=E_{p}$ pour tout $p$.

On sait que pour chaque $p$, il existe $a_{p}$ et $b_{p} \in c_{p}$ tcls que $a_{p} E_{p} \subset M_{p} \subset b_{p} E_{p}$. On peut choisir $a_{p}=b_{p}=1$ pour presque tout $p$. Dans les autres cas, on peut choisir une puissance convenable de $p$.

Posons $a=\prod_{p \in \mathbb{F}} a_{p}$ et $b=\prod_{p \in ?} b_{p}$; cela a un sens, et de plus, $a E_{p} \subset M_{p} \subset b E_{p}$ pour tout $p$. On en déduit que

$$
a N \subset M \subset b N
$$

car nous savons que $M=\bigcap_{p e}\left(M_{,}, \cap V\right)$. Donc $N$ est un réseau, en vertu du lemme 2.8 .

$N$ est inclus dans $E_{p}$, donc $N_{p} \subset L_{p}$ pour tout $p$.

Soit maintenant $x \in E_{p}$, ct supposons que $N=e_{1} \odot \cdots \odot e_{n}: x$ peut done s'écrire $\sum_{i=1}^{n} a_{i} e_{i}$, avec des $a_{i} \in Q_{p}$. Or, $\ldots$ est dense dans $\ldots .$, , donc pour chaque $i$, on peut écrire $a_{i}=a_{i}^{\prime}+\frac{a_{i}^{\prime \prime}}{p^{m}}$, avec $a_{i}^{\prime} \in \mathbb{Z}_{p}, a_{i}^{\prime \prime} \in \mathbb{Z}$ et $m \in[$ I.

Soit alors $x=x^{\prime}+x^{\prime \prime}$, avec $x^{\prime}=\sum_{i=1}^{n} a_{i}^{\prime} e_{i}$ et $x^{\prime \prime}=\sum_{i=1}^{n} \frac{a_{i}^{\prime \prime}}{p^{m}} c_{i}$. Par construction, on a $x^{\prime} \in N_{p}$. De plus, $x^{\prime \prime} \in N \subset N_{p}$, car :

i) $x^{\prime \prime} \in V$ et $x^{\prime \prime} \in N_{q} \subset L_{q}$ pour tout $q \neq p$.

ii) Puisque $N_{p} \subset E_{p}$, on a $x^{\prime \prime}=x-x^{\prime} \in E_{p}$.

On obtient alors que

$$
x^{\prime \prime} \in \bigcap_{q \in}\left(E_{q} \cap V\right)=N
$$

et on a bien $x=x^{\prime}+x^{\prime \prime} \in N_{p}$. 


\section{Définition 2.15}

Soit $(V, \beta)$, un -espace vectoriel bilinéaire ou quadratique non dégénéré. On définit :

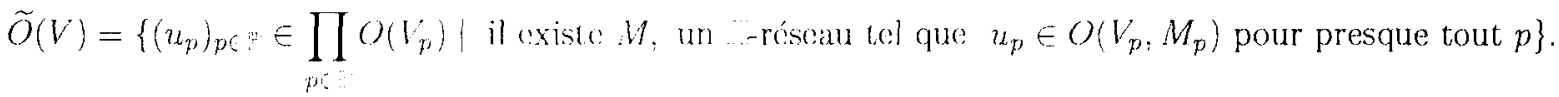

Remarquons que si cola est vrai pour un róscau $M$, c'est vrai pour tout autre réseau $N$, car $N_{p}=M_{p}$ pour presque tout $p$. Ainsi ce groupe ne dépend que de $V$.

Soit $N$, un sous-réseau de $M$; on définit aussi :

$$
\widetilde{O}(V, M / N)=\prod_{p \in \in^{t}} O\left(V_{p}, M_{p} / N_{p}\right)
$$

Nous allons montrer que $\tilde{\mathscr{Y}}=\{\tilde{O}(V, M / a M) \mid M \in \mathscr{R}$ et $c \in \cdots\}$ munit $\tilde{O}(V)$ d'une structure congruentielle.

\section{Lemme 2.16}

Soient $\widetilde{O}(V, M / a M)$ et $\widetilde{O}(V, N / b N)$; il existe $c \in \cdots$ tel que

$$
\widetilde{O}(V, N / a N) \subset \widetilde{O}(V, M / a M) \cap \widetilde{O}(V, N / b N)
$$

\section{Dérnonstration :}

On sait qu'il existe $P \in, P$ átant de cardinal fini, tel que $M_{p}=N_{p}, \forall p \in=\backslash P$.

Si $p \in P$ : Il existe $r_{p}$ et $s_{p}$ des puissances positives de $p$, telles que $s_{p} N_{p} \subset r_{p} M_{p} \subset N_{p}$. On vérifie facilement que

$$
O\left(V_{p}, N_{p} / s_{p} r_{p} a b N_{p}\right) \subset O\left(V_{p}, M_{p} / a M_{p}\right) \cap O\left(V_{p}, N_{p} / b N_{p}\right)
$$

Posors $c=\left(\prod_{q \in P} s_{q} r_{q}\right) a b ;$ on a que $c N_{p} \subset s_{p} r_{p} a b N_{p}$. On obtient donc:

$$
O\left(V_{p}, c N_{p}\right) \subset O\left(V_{p}, N_{p} / s_{p} r_{p} a b N_{p}\right) .
$$

Si $p \notin P$ : Dans ce cas, nous avons $M_{p}=N_{p}$, et clairement

$$
O\left(V_{p}, \ldots N_{p}\right) \subset O\left(V_{p}, M_{p} / a M_{p}\right) \cap O\left(V_{p}, N_{p} / b N_{p}\right),
$$

c'est à dire que

$$
\widetilde{O}(V, N / c N) \subset \widetilde{O}(V, M / a M) \cap \widetilde{O}(V, N / b N)
$$

\section{Lernme 2.17}

Soient $\widetilde{O}(V, M / a M) \subset O(V, N / b N)$; alors

$$
[O(V, M / b M): O(V, N / a N)]<\infty
$$

\section{Démonstration :}

Comme lors de la proposition 2.11, on voit facilement qu'il existo $r \in \Sigma$ tel que

$$
\widetilde{O}(V, N / r N) \subset \widetilde{O}(V, M / a M) \subset \widetilde{O}(V, N / b N)
$$


Il existe $P \subset \mathbb{P}$ fini tel que $r N_{p}=b N_{p}=N_{p}$, sauf si $p \in P$. Posons

$$
\begin{aligned}
\varphi: \widetilde{O}(V, N / b N) & \longrightarrow \prod_{p \in P} \mathscr{L}\left(N_{p} / r N_{p}\right) \\
\left(u_{p}\right)_{p \in \mathbb{P}} & \longrightarrow \prod_{p \in P} \bar{u}: N_{p} / r N_{p} \longrightarrow N_{p} / r N_{p} \\
& x_{p}+r N_{p} \longrightarrow u_{p}\left(x_{p}\right)+r N_{p} .
\end{aligned}
$$

$\varphi$ est clairement un homomorphisme de groupe. Regardons son noyau :

soient $\left(u_{p}\right)_{p \in \mathbb{P}}$ et $\left(v_{p}\right)_{p \in ?} \in \widetilde{O}(V, N / r N)$, tels que $\varphi\left(\left(u_{p}\right)_{p \in \mathbb{P}}\right)=\varphi\left(\left(v_{p}\right)_{p \in \mathbb{P}}\right)$. Cela est équivalent à l'égalité suivante :

$$
\prod_{p \in P^{p}} u_{p}\left(x_{p}\right)+r N_{p}=\prod_{p \in P^{p}} v_{p}\left(x_{p}\right)+r N_{p} \quad \forall\left(x_{p}\right)_{p \in \mathbb{P}} \in \prod_{p \in p} N_{p} .
$$

C'est aussi équivalent au lait que $u_{p}{ }^{1} v_{p}\left(x_{p}\right)-x_{p} \in r N_{p}$ quels que soient $p \in P$ et $x_{p} \in N_{p}$. Autrement dit, nous avons que

$$
\left(u_{p}\right)_{p}, l_{p},\left(u_{p}\right)_{p, p} \in \prod_{p} O\left(V_{p}, N_{p} / r N_{p}\right)
$$

et, comme les promicrs hors de P ne nous cmbelent pas, on trouve

$$
\left(u_{p}\right)_{p \in \mathbb{E}}\left(v_{p}\right)_{p \in C} \in \widetilde{O}(V, N / r N)
$$

Puisque $\prod_{p \in P} \mathscr{L}\left(N_{p} / r N_{p}\right)$ est firit, on conclut.

\section{Lemme 2.18}

Soient $u\left(=\left(u_{p}\right)_{p \in \mathbb{E}}\right) \in \widetilde{O}(V)$ et $\widetilde{O}(V, M / a M) \in \widetilde{G} ;$ alors

$$
u \tilde{O}(V, M / a M) u^{1}=\tilde{O}(V, u(M) / c \cdot u(M))
$$

où $u(M)$ est un réseau, tel que $u(M)_{p}=u_{p}\left(M_{p}\right)$; il cxiste et il est unique en vertu du lemme 2.14 .

\section{Démonstration :}

Soient $\omega \in \widetilde{O}(V, M / c M)$ et $p \in \mathbb{H}$; alors

$$
u_{p} \omega_{p} u_{p}{ }^{1}\left(u\left(M_{p}\right)\right)=u_{p} \omega_{p}\left(M_{p}\right)=u_{p}\left(M_{p}\right) .
$$

De plus, soit $m_{p} \in M_{p} ;$ on a :

$$
u_{p} \dot{\omega}_{p}, u_{p},{ }^{1}\left(u_{p}\left(m_{p}\right)\right)=u_{p}\left(\omega\left(m_{p}\right)-m_{p_{p}}\right) \in \omega_{p}\left(M_{p}\right)
$$

ce qui est équivalent à $\omega_{p}\left(m_{p}\right)-m_{p} \in c M_{p}$.

\section{Théoròme 2.19}

Le groupe orthogonal adélique $\widetilde{O}(V)$ est muni par $\mathscr{Y}$ d'une structure congruentielle. De plus, $(G, \mathscr{G})$ est unimodulaire.

\section{Démonstration :}

Le fait que $\mathscr{Z}$ soit une stucture congruentielle découle directement des lemmes 2.16, 2.17 et 2.18.

Soit maintenant $u \in \widetilde{O}(V)$. Nous savons que $\mu(E \cdot u)=c(u) \mu(E)$ pour tout $E \in \mathscr{E}$. Soit $N$ un réseau. Par définition, $u_{p} \in O\left(V_{p}, N_{p}\right)$ pour presque tout $p$. Donc, $u_{p}\left(N_{p}\right)=N_{p}$, sauf pour $p \in S$ fini. Posons

$$
u_{p}^{\prime \prime}=\left\{\begin{array}{ll}
i d_{V_{p}} & \text { si } p \in P \\
u_{p} & \text { si } p \notin P
\end{array} \quad \text { et } \quad u_{p}^{\prime}= \begin{cases}i d_{V_{p}} & \text { si } p \notin P \\
u_{p} & \text { si } p \in P\end{cases}\right.
$$




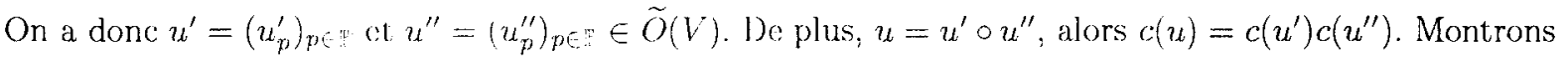
que $c\left(u^{\prime}\right)=c\left(u^{\prime \prime}\right)=1$.

$u^{\prime}$ est l'identité presque partout, et pour les premicrs de $P$, nous savons que $u_{p}$ est un produit de réflexions. On obtient, par un même raisomement que pour le théorème 2.13 , que $c\left(u^{\prime}\right)=1$.

Par constuction, $u^{\prime \prime} \in \widetilde{O}(V, N)$. Puisque pour tout $E \in \mathscr{E}, \mu\left(E \cdot u^{\prime \prime}\right)=c\left(u^{\prime \prime}\right) \mu(E)$, cette égalité est vraie en particulier si $E=\widetilde{O}(V, N)$, or on a que $E \cdot u^{\prime \prime}=E$ dans ce cas là. Donc $c\left(u^{\prime \prime}\right)=1$, puisque $\mu(E) \neq 0$.

D. Lien entre $O(V)$ et $\widetilde{O(V)}$.

Pour ce paragraphe, ainsi que pour tout le reste du chapitre, on se fixe un Q-espace vectoriel $V$ de dimension $n$ et muni d'une forme bilinćaire ou d'une forme quadratique non dégénérée et définie positive.

Remarquons tout d'abord que $O(V)$ peut être vu comme un sous-groupe de $\widetilde{O}(V)$. En effet, soient $u \in O(V)$ et $M$ un réscalu de $V: u_{p}=u \Leftrightarrow i d{ }_{n}$ est bien un élément de $O\left(V_{p}\right)$ pour tout $p$. De plus, $u_{p}\left(M_{p}\right)=u(M)_{p}$ ne differe de $M_{p}$ que prour un nombre fini de $p$.

\section{Lermme 2.20}

Soient $N$ un - réseau do $V$ a $A_{p} \in \mathscr{E}_{\nu\left(V_{p}\right)}$ pour tout $p \in \mathbb{E}$, tel que $A_{p}=O\left(V_{p}, N_{p}\right)$ pour presque tout $p ;$ alors

$$
\prod_{p \in \mathbb{P}} A_{p} \in \mathscr{E}_{\widetilde{O}(V)}
$$

\section{Démonstration :}

Soit $P=\left\{p \mid A_{p} \neq O\left(V_{p}, N_{p}\right)\right\}$ de cardinal fini. Pour chaque $p \in P$, on a $A_{p}=\bigsqcup_{i} x_{p_{i}} K_{p}$ où $K_{p}=$ $O\left(V_{p}, N_{p}^{\prime} / c_{p} N_{p}^{\prime}\right)$ et où $N_{p}^{\prime}$ est un ${ }^{-}{ }_{p}$-réscau de $V_{p}$. Comme toujours, on peut supposer que $c_{p}$ est une puissance convenable do $p$. Par le lemmo 2.14 , il existe un unique - - réseau $M$ tel que $M_{p}=N_{p}^{\prime}$ si $p \in P$, et $M_{p}=N_{p}$ si $p \notin P$.

Posons $K_{p}=A_{p}$ si $p \notin P$. On obticm:

$$
\prod_{p \in \varrho} K_{p}=\tilde{O}(V, M / c M) \in \mathscr{F}_{\widetilde{O}(V)} \text { où } c=\prod_{p \in P} c_{p}
$$

Et done, $\prod_{p \in \mathbb{P}} A_{p}$ est unc réurion finic de classes à gauche modulo $\prod_{p \in \mathbb{P}} K_{p}$.

\section{Lemme 2.21}

Soit $N$ un $\cdots$ - réseau de $V$. Pour toul $p \in$, fixons-nous $\mu_{p}$, une mesure de Haar sur $O\left(V_{p}\right)$, et $\mu$ sur $\widetilde{O}(V)$. Supposons que

$$
\prod_{p \in \mathbb{E}} \mu_{p}\left(O\left(V_{p}, N_{p}\right)\right)
$$

converge absolument. Cette convergence est indépendante du réseau $N$ choisi.

Alors, il existe une constante $c$ positive, telle que pour toute famille $\left(A_{p}\right)_{p \in \mathscr{z}}$, avec $A_{p} \in \mathscr{C}_{O\left(V_{p}\right)}$ quel que soit $p$ premier et $A_{p}=O\left(V_{p}, N_{p}\right)$ saul pour $p \in P$ fini, on ail :

$$
\prod_{p} \mu_{p}\left(A_{p}\right)=c \mu\left(\prod_{p \in \mathbb{p}} A_{p}\right)
$$

Le membre de droile de coto égalité est bion défini en vertu du lemme précédent. 
On en déduit que si les $\mu_{p}$ sont tels que $\prod_{p \in \mathbb{R}} \mu_{p}$ converge, alors $\prod_{p \in \mathbb{R}} \mu_{p}$ est une mesure sur $\widetilde{O}(V)$.

\section{Démonstration :}

Posons $c>0$ tel que

$$
c \mu\left(\prod_{p \in E} O\left(V_{p}, N_{p}\right)\right)=\prod_{p \in \mathbb{Z}} \mu_{p}\left(O\left(V_{p}, N_{p}\right)\right) .
$$

Fixons-nous une famille $\left(A_{p}\right)_{p \in z}$, telle que $A_{p}=O\left(V_{p}, N_{p}\right)$, sauf pour $p \in P=\left\{p_{1}, \ldots, p_{s}\right\}$. Posons

$$
\begin{aligned}
\xi_{p_{1}}: \mathscr{E}_{O\left(V_{p_{1}}\right)} & \longrightarrow \mathbb{F}_{1} \\
B & \longmapsto \mu_{p_{1}}(B) \cdot \prod_{q \in \mathbb{P} \backslash\left\{p_{1}\right\}} \mu_{q}\left(O\left(V_{q}, N_{q}\right)\right)
\end{aligned}
$$

et

$$
\begin{aligned}
\bar{\xi}_{p_{1}}: \mathscr{E}_{O\left(v_{p_{1}}\right)} & \longrightarrow \mu\left(B \times \prod_{x \in\left\{p_{1}\right\}}\left(O\left(V_{q}, N_{q_{1}}\right)\right) .\right. \\
B & \longmapsto \mu(1)
\end{aligned}
$$

$\xi_{p_{1}}$ at $\bar{\xi}_{p_{1}}$ sont clairement des mesures sur ()$\left(V_{p_{1}}\right)$. Il existe donc $c_{p_{1}}$ positil tel que $\xi_{p_{1}}=c_{p_{1}} \bar{\xi}_{p_{1}}$. Cette égalité est vraie, en particulier si $B=O\left(V_{p_{1}}, N_{p_{1}}\right)$. On en déduit alors que $c_{p_{1}}=c$; donc l'égalité suivante est vérifiée :

$$
c \mu\left(A_{p_{1}} \times \prod_{q \in\left\{p_{1}\right\}}\left(O\left(V_{q}, N_{q}\right)\right)=\mu_{p_{1}}\left(A_{p_{1}}\right) \cdot \prod_{q \in \bigoplus \backslash\left\{p_{1}\right\}} \mu_{q}\left(O\left(V_{q}, N_{q}\right)\right) .\right.
$$

Soient maintenarıt

$$
\begin{aligned}
\xi_{p_{2}}: \mathscr{E}_{O\left(v_{p_{2}}\right)} & \longrightarrow \ldots \\
B & \longmapsto \mu_{p_{2}}(B) \cdot \mu_{p_{1}}\left(A_{p_{1}}\right) \cdot \prod_{q \subset \backslash\left\{p_{1}, p_{2}\right\}} \mu_{q}\left(O\left(V_{q}, N_{q}\right)\right)
\end{aligned}
$$

et

$$
\begin{aligned}
\bar{\xi}_{p_{2}}: \bar{E}_{\left(\gamma\left(V_{p_{2}}\right)\right.} & \longrightarrow \cdots \\
B & \longmapsto \mu\left(B \times A_{p_{1}} \times \prod_{q \subset \backslash\left\{p_{1}, p_{2}\right\}}\left(O\left(V_{q}, N_{q}\right)\right) .\right.
\end{aligned}
$$

De nouveau, il existe $c_{p_{2}}$, tel que $\xi_{p_{2}}=c_{p_{1}} \bar{\xi}_{p_{2}}$; on trouve alors que $c_{p_{2}}=c$. On refait le même procédé jusqu'à $p_{s}$, et on tromve:

$$
\mu\left(\prod_{p c} A_{p}\right)=\prod_{p c} \mu_{p}\left(A_{p}\right)
$$

\section{E. Domaine fondamental et masse.}

\section{Définition 2.22}

Soient $(G, \mathscr{J})$ un groupe muni d'une structure congruentielle, $O$ un sous-groupe de $G$, et $M \in \mathscr{E}$. Si $F \in \mathscr{E}$ est tel que

$$
O M=\bigsqcup_{x \in O} x F
$$

$F$ est appelé domaine fondamental pour $M$ relatif à $O$.

\section{Lemrne 2.23}

Soient $G$ un groupe, $H_{1}, H_{2}$ deux sous-groupes do $C$, at $x, y \in C$. Si $H_{1} x H_{2} \cap H_{1} y H_{2} \neq \emptyset$, alors

$$
H_{1} x H_{2}=H_{1} y H_{2}
$$

\section{Démonstration :}

C'est une vérification évidente. 


\section{Théorème 2.24}

Sil existe $K \in \mathbb{F}$ tel que $O \cap x K x^{1}=\{1\} \forall x \in G$, alors tout $M \in \mathscr{E}$ possède uri domaine fondamental relatif à $O$. De plus, si $F_{1}$ or $F_{2}$ somt deux domaines fondamentaux pour $M$, on a $\mu\left(F_{1}\right)=\mu\left(F_{2}\right)$.

\section{Démonstration :}

Soit $M \in \mathscr{E} ; M$ est une réunion finie de classes à gauche d'un certain $L \in \mathscr{Z}$ tel que $L \subset K$. On a

$$
M=\bigsqcup_{i=1}^{i} x_{i} L
$$

et, grâce au lemme précédent,

$$
O M=\bigcup_{i=1}^{t} O x_{i} L=\bigsqcup_{j=1}^{s} O y_{j} L
$$

où $\left\{y_{1}, \ldots, y_{s}\right\} \subset\left\{x_{1}, \ldots, x_{t}\right\}$. Posons $F=\bigsqcup_{j=1}^{s} y_{j} L$. On a déjà que $O M=\bigcup_{x \in O} x F$. Il nous reste à voir que la réunion est disjointe:

Soit $v, u \in O, v \neq u$. Supposons que uf $7 v: \% \neq$. On a alors:

$$
\left(\bigsqcup_{j=1}^{s} u y_{j} L\right) \cap\left(\bigsqcup_{j=1}^{s} u y_{j} L\right) \neq \emptyset
$$

Donc, puisque les $O y_{j} L$ sont disjoints, il existe $y_{i}$ et $\ell_{1}, \ell_{2} \in L$ tels que $u y_{2} \ell_{1}=v y_{i} \ell_{2}$. Mais cela n'est pas possible, car sinon on aurait $1 \neq u^{1} v=y_{i} \ell_{1} \ell_{2}{ }^{1} y_{i} \in y_{2} L_{2} y_{i}{ }^{1} \subset y_{i} K y_{i}^{-1}$, ce qui est contraire à l'hypothèse. Suposons maintenant quil oxiste $F_{1}$ at $F_{2} \in \mathscr{E}$ tols que

$$
\bigsqcup_{x \in 0} x F_{1}=\bigsqcup_{x \in 0} x F_{2}
$$

Il existe $K \in \mathscr{F}$ tel que

$$
r_{1}=\bigsqcup_{i=1}^{n} x_{i} K \text { et } r_{2}=\bigsqcup_{j=1}^{m} y_{j} K
$$

On trouve que

$$
\bigsqcup_{i=1}^{n} O x_{i} K=\bigsqcup_{j=1}^{m} O y_{j} K .
$$

Le lemme précédent. nous dit alors que $m=n$, donc $\mu\left(F_{1}\right)=\mu\left(F_{2}\right)$.

\section{Définition 2.25}

Soient $(G, \mathscr{Y})$ un groupe muni d'une structure congruenticlle, $\mu$ la mesure associée, $O$ un sous-groupe de $C, M \in \mathscr{E}$, of $F$ un dombine fondamental pour $M$ relatif à $O$.

On pose

$$
\left.m(0)^{(M)}\right):=\mu(r)
$$

où $O \bigcup^{O M}=\{O x \mid x \in O M\}$.

On en déduit tout de suite kes propriétés suivantes:

\section{Proposition 2.26}

a) $m\left(o \Upsilon^{O M}\right)>0$

b) Soient $M_{1}$ et $M_{2} \in E$ tols que $O M_{1} \cap O M_{2}=0$. On a :

$$
\left.m\left(O \bigcup^{\left(M_{1}-M_{2}\right)}\right)=m(o)^{O M_{1}}\right)+m\left(o \backslash^{O M_{2}}\right) .
$$

\section{Démonstration :}

Evident. 


\section{Proposition 2.27}

Soient $N$ un Z-réseau de $V$, et $p$ un nombre premier différent de 2 . Alors

$$
O(V) \cap \widetilde{O}(V, N / p N)=\{1\}
$$

\section{Démonstration :}

Soit $1 \neq u \in O(V) \cap \widetilde{O}(V, N / p N)$. En particulicr, $u \in \widetilde{O}(V, N) \cap O(V, N)$ d'après le lemme 2.14. Nous savons que $O(V, N)$ ust fini puisque $\beta$ cst définic positive; u est alors d'ordre fini. Quitte à prendre une puissance convenable, on peut supposer que $u$ est d'ordre $q \in \mathbb{P}$.

Relativement à une $-\cdots$-base de $N, u$ cst représentée par $A$ une matrice à coefficients entiers. Puisque $u \neq 1$, $A=I+\widetilde{U}$, où $I$ est la matrice unité, et $\bar{U}$ une matrice quelconque non mulle. Or, pour tout $x \in N$, on a $u(x)-x \in p N$, donc $p$ divise $\vec{U}$. Finalement,

$$
A=I+p^{m} U
$$

avec $p \nmid X$ et $m \geq 1$. On obtiont alors :

$$
I=\left(I+p^{m} U\right)^{q}=I+q p^{m} U+\cdots \text { Cette somme contient au moins } 3 \text { termes. }
$$

i) Si $p \neq q$, on a $: q p^{m} U \equiv 0 \bmod p^{2 m}$ donc $U \equiv 0 \bmod p^{m} ;$ ce qui vout dire que $p$ divise $U$, ce qui est absurde.

ii) Si $p=q$, on obtient $p^{m+1} \equiv 0 \bmod p^{2 m+1}$, car

$$
I=I+r^{m: 1} U+\left(\begin{array}{c}
p \\
2
\end{array}\right) p^{2 m} U^{2}+\cdots
$$

el $p$ divise $\left(\begin{array}{l}p \\ 2\end{array}\right)$ puisqu'il col different do 2 . l'inalement, on trouve que $U \equiv 0 \bmod p^{m}$ qui est aussi absurde que tout à l'houre.

\section{Théorème 2.28}

Le groupe adćlique $\widetilde{O}(V)$ possède un domaine fondamental relatif à $O(V)$. Il est done permis de parler de $\left.m(o(V))^{\tilde{O}(V)}\right)$.

\section{Démonstration :}

Soit $u \in \widetilde{O}(V)$; alors

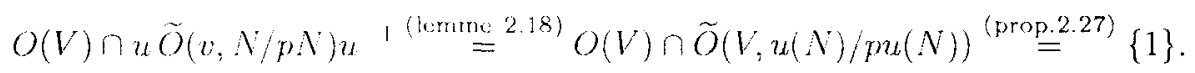

Et on conclut, grâce au théorème 2.24 .

\section{Théorèrne 2.29}

Soient $M$ un réseau quadrat ique pour lia forme q $=\beta(x, x)$, w M l'ensomble des ...-réseaux de $V$ qui sont dans le même genre que $M$. Lapplicalion

$$
\begin{aligned}
\sigma & \sigma(V) / \widetilde{O}(M) \longrightarrow \mathscr{M} \\
& u \bmod \widetilde{O}(M) \longmapsto u(M)
\end{aligned}
$$

est bien délinie, et de plus, ollo est bijective. 


\section{Dérnonstration :}

Soit

$$
\begin{aligned}
& \psi: \widetilde{O}(V) \longrightarrow \mathscr{M} \\
& u \longmapsto u(M) .
\end{aligned}
$$

Rappelons que $u=(u)_{p \in \text { : }}$, et que $u(M)$ est l'unique réseau, lel que $u(M)_{p}=u_{p}\left(M_{p}\right)$. 11 s'ensuit que $u(M)$ et $M$ sont dans le même genre.

Soient maintenant $u$ et $u^{\prime} \in \widetilde{O}(V)$, tels que $u u^{-1} \in \widetilde{O}(M)$. On a donc que $u_{p}^{-1} u_{p}^{\prime} \in O\left(M_{p}\right) \forall p$. Cela est équivalent à :

$$
u_{p}\left(M_{p}\right)=u_{p}^{\prime}\left(M_{p}\right) \forall p \in \mathbb{P} .
$$

D'où, $u^{\prime}(M)=u(M)$, ce qui veut dire que l'application $\varphi$ est bien définie.

Soit $M^{\prime}$ un réseau dans le même genre que $M$, il existe $u \in \widetilde{O}(V)$ tel que $u_{p}\left(M_{p}\right)=M_{p}^{\prime}$; grâce au lemrne 2.14 , cela veut dire que $u(M)=M^{\prime}$. Done $\varphi$ est surjective.

Finalement, $\varphi$ est injective, car si $u \widetilde{O}(M)$ et $u^{\prime} \widetilde{O}(M)$ sont tels que $u_{p}^{\prime}\left(M_{p}\right)=u_{p}\left(M_{p}\right) \forall p$, alors, cela est équivalent au fait que $u_{p}^{1} u_{p}^{\prime} \in \widetilde{O}(K)$, donc que $u \widetilde{O}(M)=u^{\prime} \widetilde{O}(M)$.

\section{Corollaire 2.30}

Soient $a \in z, M$ un réseau quadratique pour $q=\beta(x, x)$ respectivement pour $q$ si $V$ est quadratique, et $K=\widetilde{O}(V, M / a M)$. On a que $O(V)$ est une réunion finie de classes doubles $O(V) u_{i} K$.

\section{Dérnonstration :}

Il suffit de démontrer ce résultat pour $K=\widetilde{O}(M)$, war los autres sont d’indice fini par rapport à lui. Posons

$$
\begin{aligned}
u: \partial(v) \backslash \widetilde{\partial(V)} / \partial(M) & \longrightarrow \overline{\mathscr{M}} \\
O(V) u \widetilde{O}(M) & \longmapsto \overline{u(M)}
\end{aligned}
$$

Soient $u$ et $v \in \widetilde{O}(V)$ tels que $O(V) u \widetilde{O}(M)=O(V) v \widetilde{O}(M)$. Cette égalité est équivalente à l'existence de $\tau \in O(V)$ et de $\xi \in \widetilde{O}(M)$ tels que $v=\tau u \xi$; qui est équivalent à $v(M)=\tau(u(M))$; ce qui veut dire que $v(M)$ et $u(M)$ sont isornorphes, ou encore que $\overline{v(M)}=\overline{u(M)}$.

Done $\psi$ est bien définie et injective.

Soit $M^{\prime} \in \overline{M^{\prime}}$ qui est une classo d’ismorphismes dans le genre de $M$. Le thécorène précédent nous donne l'existence d'un $u \in \widetilde{O}(V)$ tel que $\gamma(u \widetilde{O(M)})=V^{\prime}$, ce qui veut dire que $\psi(O(V) u \widetilde{O}(M))=\overline{M^{\prime}}$.

\section{F. Représentations.}

\section{Définition 2.31}

Soient $\left(L, \beta_{L}\right)$ et $\left(M, \beta_{M}\right)$ deux $\ldots$-modules bilinéaires non dégénérés, libres de rang finis. Un homomorphisme de 2 module $u$ de $L$ dans $M$ tel que $q_{M}(u(x))=q_{L}(x) \forall x \in L$ où $q_{M}=\beta_{M}(x, x)$ et $q_{L}=\beta_{L}(x, x)$ est appelé représentation do L par $M$.

Si $L$ et $M$ sont des - -modules quadratiques non dégénérés, on défini les représentations de manière identique.

Remarquons que $u$ est injective puisque $L$ est non dégénóré.

\section{Définitions 2.32}

Soient $L, M$ et $M^{\prime}$ des modules bilinćaires ou quadratiques non dégénérés; soient de plus $u: L \rightarrow M$ et $u^{\prime}: L \rightarrow M^{\prime}$ deux représontations. On dit quo $u$, $u^{\prime}$ sont équivalents, et on écrit $u_{1} \simeq u_{2}$, s'il existe 
un isomorphisme $f$ de $M$ dans $M^{\prime}$ lol que lo diagramme

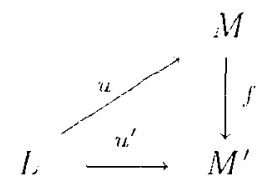

commute. Il en découle en particulier que $M_{1}$ et $M_{2}$ sont équivalents.

On dit que $u, u^{\prime}$ sont de mème genre, et on écrit $u_{1} \sim u_{2}$, si pour tout $p$ premier, il existe un isomorphisme $f_{p}: M_{p} \longrightarrow M_{p}^{\prime}$ laisant commuter le diagramme suivant :

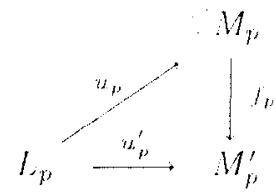

$M$ et $M^{\prime}$ sont en particulier dans le même genre.

\section{Lemme 2.33}

Supposons que $\beta_{M}$ soil definio posilive.

1) Il n'existe qu'un mombre firit de représentations de $l$ par $M$.

2) Il n'existe qu'un nombre fini de classes de représentations de $I$. par des ...-modules du genre de $M$.

\section{Démonstration :}

1): Soit $a \in Z$; posons $S_{a}=\{y \in M \mid q M(y)=a\}$. Le cardinal de $S_{a}$ est lini.

En effet, posons $V_{F}=M \otimes K ; q_{M}$ s'étond naturelloment sur $V_{E}$. Soit $v \in V_{B}$; posons

$$
\|v\|=\sqrt{q_{M}(v)}
$$

$\|\cdot\|$ est une norme sur $V$. Puisque loute les normes sont équivalentes sur $V$, on a que $M$ est discret, et donc $S_{a}=B\left(0, a^{2}\right) \cap M$ s.st lini.

Si on veut que $q_{M}(u(x))=q_{1}(x)$, on n'a donc qu'un nombre fini de choix pour $u(x)$; el comme $u$ est défini en combissant les images des vectems de bases de $/$ qui sont en nombre lini, on conclut.

2) : On a vu dans le premier chapilre quil n'y a qu'un nombre lini de elasses disomorphisme de Z-module bilinéaire ou quadratique dans un genre domé. Soinent done $M=M_{1}, M_{2}, \ldots, M_{k}$ des représentants des classes d'équivalences dars lo genre do M. P’osons

$$
P=\bigcup_{j}^{k}\left\{u_{j} \mid u_{j} \text { représente } L \text { dans } M_{j}\right\} \text {. }
$$

Vu 1), nous savons que $P$ cost lini. Soit $M^{\prime}$ tel que $M^{\prime} \sim M$ et tel qu’il existe $u^{\prime}$ qui représente $L$ dans $M^{\prime}$. On sait qu'il existe $j \in ! l_{k}$ tel que $M_{j} \simeq M$. Posons $u_{j}^{\prime}: L \longrightarrow M_{j}, u_{j}=f \circ u^{\prime}$ ò̀ $f$ est l'isomorphisme entre $M^{\prime}$ et $M_{j}$. Or a alors $u_{j} \in P^{\prime}$ ot $u_{j}^{\prime} \simeq u^{\prime}$.

\section{Définition 2.34}

Soit $L$ un sous---module bilinćaire ou quadratique de $M$. L'inclusion de $L$ darns $M$ constitue ce que l'on appelle une représentalion spéciale de L par $M$. 
Remarquons que toute classe d’isomorphisme de repésentation de $L$ par $M$ contient une représentation spéciale; on a même mieux :

\section{Lemme 2.35}

Supposons que: $V=M \&{ }^{\prime} ;$ alors loute classe de représentation de $l$. par un $\cdots$-module du genre de $M$ contient une représentation speciale de $l$ par un -...-réseau de $V$

\section{Démonstration :}

Soit $M^{\prime} \sim M$. Supposons qu'il existe $u: L \longrightarrow M^{\prime}$, une représentation. Posons $V^{\prime}=M^{\prime} \otimes Q$ et $U=L \otimes$. $U$ est un sous-espace vectoriel de $V$, et $u$ engendre une application $\widetilde{u}$ de $U$ dans $V^{\prime}$. Par le théorème de Witt tel qu'il est vu dans [10, thm. 3, p. 58], $\tilde{u}$ se prolonge en $\bar{u}: V \longrightarrow V^{\prime}$, tel que

$$
\bar{u} \in O\left(V, V^{\prime}\right) \stackrel{\text { def }}{=}\left\{u: V \longrightarrow V^{\prime} \mid \beta_{V^{\prime}}(u(x), u(y))=\beta_{V}(x, y) \forall x, y \in V\right\}
$$

Posons $M^{\prime \prime}=\bar{u}^{-1}\left(M^{\prime}\right)$ et $v: I \longrightarrow M^{\prime \prime}$ tel que $v=\bar{u}^{1}$ ou. Il est clair que $v \simeq u$.

\section{Définition 2.36}

Soient $W$ un sous-espace vectoriel de $V$, et $M$ un réseau de $V$. Posons $U=W$ :

On définit,

$$
O(W, M)=\left\{v \in O(W) \mid\left(v \oplus \mathrm{id}_{l^{\prime}}\right) \in O(V, M)\right\}
$$

On pose aussi

$\widetilde{O}(W)=\left\{\left(v_{p}\right)_{p e r} \in \prod_{p} O\left(W_{p}\right) \mid\right.$ il cxiste $M$ un réscau de $V$ tel que $v_{p} \in O\left(W_{p}, M_{p}\right)$ pour presque tout $\left.p\right\}$

Et on définit

$$
\tilde{O}(W, M)=\left\{\left(v_{p}\right)_{p} \in \tilde{O}(W) \mid\left(v_{p}, \mathrm{id}_{i_{p}}\right)\left(M_{p}\right)=M_{p}, \forall p \in \mathbb{H}\right\}=\prod_{p \in \mathbb{P}} O\left(W_{p}, M_{p}\right)
$$

Ces ensembles sont clairement des groupes.

\section{Remarque :}

Si $W$ est vu comme espace vectoriel "tout nu", lors de la délinition 2.15 , nous avions défini

$\widetilde{O}(W)=\left\{\left(v_{p}\right)_{p \in \mathbb{R}} \in \prod_{p} O\left(W_{p}\right) \mid\right.$ il existe $M$ ur réscan de $W$ tel que $v_{p} \in O\left(W_{p}, M_{p}\right)$ pour presque tout $\left.p\right\}$

Une petite verification nous perrnet de voir que cos deux groupes coincident, done qu'il n'y a pas d'abus de notation.

\section{Proposition 2.37}

On sait que $O(W)$ peut etre muni d'une stucture congruerticlle. De plus, on a que $O(W, M)$ est un ensemble de congruence do $O(W)$.

\section{Démonstration :}

Soit $p$ la projection de $V$ sur $W$ le long de $V$. On a que $p(M)$ et $W \cap M$ sont des réseaux de $W$. Il existe donc $a \in \mathcal{W}$ tel que $a \cdot p(M) \subset W \cap M$. Nors, on a :

$$
O(W, p(M) / a P(M)) \subset O(W, M) \subset O(W, p(M))
$$


En prouvant ces inclusions, on prouve la propesition gráce à ce que l'on sait sur les sous-groupes de congruence.

Prouvons la première inclusion :

Soit $v \in O(W, p(M) / a \cdot p(M))$. Il suflit de voir que $\left(v \oplus \mathrm{id}_{U}\right)(M)=M$. Soit $m \in M$. On a que $m=$ $m_{U}+m_{W} \in U \ominus W$. Par hypothese, on a :

$$
v\left(m_{W}\right)-m_{W} \in a p(M) \subset W \cap M
$$

Par définition, $\left(v \ominus \mathrm{id}_{\ell}\right)\left(m_{l}\right)=v\left(m_{W}\right)+m_{\ell}$. Or, comme $m \in M$, il s'ensuit que $\left(v\left(m_{W}\right)-m_{W}\right)+\left(m_{W}+\right.$ $\left.m_{U}\right) \in M$. Donc, $\left(v \oplus \mathrm{id}_{U}\right)(M) \subset M$. En faisant le même raisomement pour $\left(v^{\cdots-1} \oplus \mathrm{id}_{U}\right)$, on conclut. Il nous reste à montrer la deuxieme inclusion:

Soit $v \in O(W, M)$. Posons $p^{\prime}$ la projection sur $U$ le long de $W$. On a :

$$
p(M) \boxplus p^{\prime}(M)=M=\left(v \oplus \mathrm{id}_{U}\right)(M)=v(p(M)) \boxplus p^{\prime}(M) .
$$

Donc $v(p(M))=p(M)$.

\section{Proposition 2.38}

Le groupe $\widetilde{O}(W, M)$ ast un consemble de congruence pour $\widetilde{O}(W)$.

\section{Démonstration :}

La démonstration est similaire à celle de la proposition précédento.

\section{Proposition 2.39}

Soient $L \subset M$ deux -modules bilinéaires ou quadratiques libres de rang fini. Posons $V=M \otimes O$, $U=L \otimes$, et $W=U$. Alors, le nombre de représentations de $L$ par $M$ qui sont dans la même classe d'isomorphismes que l'inclusion $j: I_{i} \longleftrightarrow M$ est l'indice $[O(V, M): O(W, M)]$.

\section{Démonstration :}

Remarquons que si $v$ est une application linciare de $l$ dans $M$, elle se prolonge naturellement en une application linéaire $\tilde{a}$ do $U$ dans $V$.

Soient $X$ l'ensemble des reprósentalions de $L$ par $M$ qui sont isomorphes à j, et $Y=O(V, M) / \approx$ où $f \approx g$ si et seulement si $\left.f\right|_{1}=\left.g\right|_{L}$. Soit

$$
\begin{aligned}
\varphi: X & \longrightarrow Y \\
v \longmapsto & \longrightarrow \overline{f_{v}} .
\end{aligned}
$$

L'application $\int_{v}$ est définic comme suit :

nous savons par hypothese que $v=j$. Il existe done f faisant commuter le diagramme suivant :

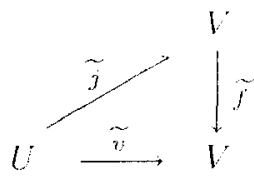

Il est clair que $\tilde{f}_{l}=v$. Cela vout dire que $\varphi$ est bien définie ol injective.

Montrons la surjectivité :

Soit $f$ un représentant d'une des dasses de $\gamma$. Posons $v=\left.f\right|_{M} \cdot j$; alors $\varphi(v)=\bar{f}$. 
D'autre part, soit $f \approx g$; cela est équivalent à $\left.f\right|_{\ell}=\left.g\right|_{\ell}$ ou encore $\left.g f^{-1}\right|_{\ell}=i d_{\ell}$. Puisque $f$ et $g$ sont dans $O(V, M)$, cela veut dire que $g \cdot f^{-1} \in O(W, M)$.

Donc $[O(V, M): O(W, M)]$ compte bien le nombre de représentations de $L$ par $M$ dans la même classe que $j$.

\section{Remarques :}

1) $\widetilde{O}(W)$ peut être vu comme un sous-groupe de $\widetilde{O}(V)$. En eftet, si $v_{p} \in O\left(W_{p}\right)$, $v_{p} \oplus \mathrm{id}_{U_{p}}$ est bien défiri sur $V_{p}$, et on a

$$
q_{p}\left(\left(u_{\gamma}, \mathrm{oid} U_{p}\right)(x)\right)=q_{p}(x)
$$

$\operatorname{car} W=U^{\perp}$.

2) Soit $\gamma$ l'ensemble des ...-réscaux $N$ de móme genre que $M$ et tels que les applications $u_{p}: M_{p} \longrightarrow N_{p}$ vérifient $\left.\overline{u_{p}}\right|_{U_{p}}=\mathrm{i} d_{\ell_{p}}$, où $\overline{u_{p}}$ est l'extension naturelle de $u_{p}$ sur $V_{p}$. Alors $\gamma$ est en bijection avec l'ensemble des représentations de $l$ par $M$ dans le môme genre que $j$.

Ce lait est plus facilo à dérmontrer qu'á énoncer.

\section{Proposition 2.40}

On a une bijection entre $\widehat{O}(W) / \widehat{O}(W, M)$ et l'ensemble des représentations de $I$ par $M$ dans le même genre que $j$.

\section{Démonstration :}

Par ce qui préecde il sulfil de voir que l'application

$$
\begin{aligned}
u: \tilde{O}(W) / \partial(W, M) & \longrightarrow \gamma \\
u \tilde{O}(W, M) & \longrightarrow\left(u \oplus \mathrm{id}_{U}\right)(M)
\end{aligned}
$$

est une bijection.

Soient $u$ et $u^{\prime} \in \widetilde{O}(W)$, tels que $u^{\prime} u^{\prime} \in \widetilde{O}(W, M)$. Cela veut dire que

$$
\left(u_{p}{ }^{1} u_{p}^{\prime}, \mathrm{id} l_{l_{s}}\right)\left(M_{p}\right)=M_{p} \forall p \in
$$

ou encore

$$
(u \odot \operatorname{id})(M)=\left(u_{p} \odot \operatorname{id}_{\ell}\right)_{y \in}(M)=\left(u_{p}^{\prime} \odot \mathrm{id}_{l_{p}}\right)_{p \in O}(M)=\left(u^{\prime} \odot \mathrm{id}_{U}\right)(M) .
$$

Donc $\psi$ est bien définie et injective.

Soit $M \in \gamma$. Il existe done $\left(v_{p} \ominus \mathrm{id}_{U_{p}}\right)_{p \in} \in \widetilde{O}(V)$ tel que $\left(v_{p} \ominus \mathrm{id}_{\iota_{p}}\right)_{p \in \Subset}(M)=M^{\prime}$.

Finalement, $\left(v_{p}\right)_{p \in \mathbb{E}} \in \widetilde{O}(W)$, et de plus, $\psi\left(\left(v_{p}\right)_{p \in \mathbb{F}}\right)=M^{\prime}$. Donc $\psi$ est surjective.

\section{Corollaire 2.41}

Le groupe $\widetilde{O}(W)$ est une réurion de dasses doubles $O(W) v_{i} \widetilde{O}(W, M)$.

\section{Démonstration :}

Posons

$$
\begin{aligned}
\theta: \partial(w) \backslash \sigma(w) / \sigma(w, M) & \longrightarrow \bar{\gamma} \\
\partial(W) v \sigma(W, M) & \longmapsto \overline{\left(v \ominus i d_{u}\right)(M)}
\end{aligned}
$$

Par un raisonnemont identique à la démonstration du corollaire 2.30 , on woil que $\theta$ est injective et bien définie. Ia proposition précédente: nous donne la surjectivité, et, puisque: $\bar{Y}$ est fini en vertu du lemme. 2.33 , on conclut.

\section{G. Formule de Siegel.}


Dans ce paragraphe, on se fixe $M$ un $\cdots$-réscan quadratique de $V, L \subset . M$ un $\cdots$-module quadratique libre, $U$ le sous-espace de $V$ engendró par $l$, et $W=U$.

\section{Lemme 2.42}

Soient $(G, \mathscr{g})$ un groupe muni d'une structure congruentielle, $K \in \mathscr{g} ; O$ un sous-groupe de $G$, et $u \in G$ tels qu'il existe $F \subset u K$ un domaine fondamental pour $u K$ relatif à $O$. Ce qui veut dire que :

$$
O_{u} K=\bigsqcup_{x \in O} x F
$$

alors, on a:

$$
u K=\bigsqcup_{y \cos u K u-1} y F
$$

\section{Démonstration :}

On a bien sûr :

$$
u K=O u K \cap u K=\bigsqcup_{x \in O}(x F \cap u K)
$$

Si $x F \cap u K \neq \emptyset$, alors $x F^{\prime} \subset u K$. En offot, supposons que $x J=u k$; puisque $F \subset u K$, on peut écrire $f=u k^{\prime}$. On a donc que $x=u k^{\prime \prime} u^{1} \in u K^{\prime} u^{-1}$. Or

$$
x F^{\prime} \subset x u K=u k^{\prime \prime} u^{-1} u K \subset u K \text {. }
$$

On obtient donc $u K=\bigsqcup_{y c(r) u K u} y F \cdot$

\section{Proposition 2.43}

Nous savons par le corollaire 2.41 qu'il existe $u_{1}, \ldots, u_{m} \in \widetilde{O(W)}$ tels que

$$
\widetilde{O}(W)=\bigsqcup_{i=1}^{m} O(W) u_{i} \widetilde{O}(W, M)
$$

Alors, on a :

$$
m\left(O(W) \bigcup^{\tilde{O}(W)}\right)=\mu(\tilde{O}(W, M)) \sum_{i=1}^{m} \frac{\left[O\left(u_{i}, M\right): O\left(W, u_{i} M\right)\right]}{\left|O\left(u_{i} M\right)\right|}
$$

où $\mu$ est la mesure sur

\section{Démonstration :}

On a :

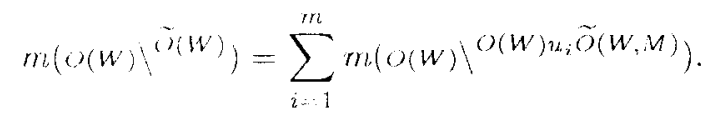

Le théorème 2.28 nous assure l'cxistence pour tout $i \in I_{m}$ d'un $F_{i} \in \mathscr{E}_{\widetilde{O}(W)}$ tel que

$$
O(W) u_{i} \tilde{O}(W, M)=\bigsqcup_{x \in O(W)} x F_{i}
$$

Le lemme précédent nous permet alors de dire:

$$
u_{i} O(W, M)=\bigsqcup_{x \in O(W)^{-} u_{i} \widetilde{O}(W, M) u_{i}^{-1}} x F_{i}
$$

On vérifie facilement que

$$
O(W) \cap u_{i} \tilde{O}(W, M)=O\left(W, u_{n}, M\right)
$$

Or, $m\left(O(W) \backslash O(W) u_{i} \widetilde{O(W, M)}\right)=\mu\left(F_{i}\right)$. Vous savons que

$$
u_{i} \widetilde{O}(W, M)=\bigsqcup_{x \in O\left(W, u_{i} M\right)} x F_{i}
$$

Donc:

$$
\mu\left(F_{i}\right)=\frac{\mu\left(u_{i}(\tilde{O}(W, M))\right.}{\left|O\left(W, u_{i}, M\right)\right|}=\frac{\mu(\widetilde{O}(W, M))}{\left|O\left(u_{i} M\right)\right|}\left[O\left(u_{i}, M\right): O\left(W, u_{i} M\right)\right]
$$




\section{Rerrarque :}

On a que $L \subset u_{i}(M) \forall i \in\left[I_{r b}\right.$, car $u_{i} \in \widetilde{O}(W)$; et on peut voir $u_{i}$ comme un élérnent de $\widetilde{O}(V)$ en prenant $u_{i_{p}} \oplus i \mathrm{~d}_{j_{p}}$. Puisque $U_{p} \supset L_{p} \forall p \in \mathbb{E}$, on a

$$
\left(u_{i_{p}} \oplus \mathrm{id}_{U_{p}}\right)\left(L_{p}\right)=L_{p} \quad \forall p \in \mathbb{F}
$$

ce qui veut dire que $u_{i}\left(L_{p}\right)=L_{p}$.

\section{Proposition 2.44}

Les inclusions $j_{i}: \quad l \longrightarrow u_{i}(M)$ constituent un systime complet de représentants des classes de représentations qui sont dans le genre de l'inclusion $j: I \longleftrightarrow M$.

\section{Démonstration :}

On a déjà que $L \smile u_{i}(M)$ est dans le même genre que $j \forall i \in I_{m}$; car le diagramme

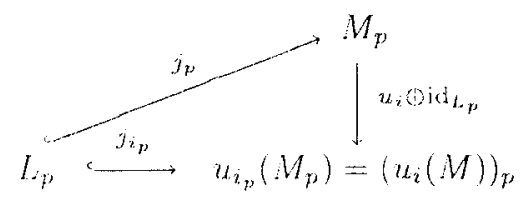

commute puisque $u_{i}(L)=L$.

Soit maintenant $u^{\prime}: L \longrightarrow M^{\prime \prime}$ du môme genre que $j$. On sait grâce au lemme 2.35 qu'il existe $u: L \longrightarrow$ $M^{\prime}$, une représentation spéciale dans la même classe que $u^{\prime}$. Donc quel que soit $p \in \mathbb{E}$, il existe $f_{p}$ tel que le diagramme

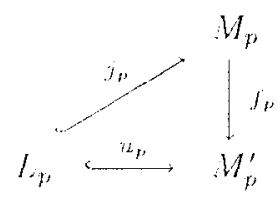

commute. Elant donmé que $j_{p}$ et $u_{p}$ sont des inclusions, on a que $\widetilde{f}_{r}: V_{p} \rightarrow V_{p}$ est telle que $\left.\widetilde{f}_{p}\right|_{U_{p}}=\mathrm{id}_{U_{p}}$. $\tilde{f}_{p}$ s'écrit alors $\int_{p}^{\prime} \Theta \mathrm{id}_{i_{p}}$ ò̀ $f_{p}^{\prime} \in O\left(W_{p}\right)$. Or, $M_{p}=M_{p}^{\prime}$ pour presque tout $p$, donc $\left(\widetilde{f}_{p}\right)_{p \in \mathbb{P}} \in \widetilde{O}(W)$; on a de plus, $(\widetilde{f})_{p \in}(M)=M^{\prime}$. Nous savons que

$$
\widetilde{O}(W)=\bigsqcup_{i=1}^{m} O(W) u_{i} \widetilde{O}(W, N)
$$

donc il existe un unique $i \in I ! m$ tel que:

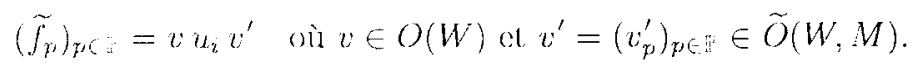

$v$ s'étend on $v \oplus$ id $\ell \in O(V)$, que nous nolerons quand meme par abus $v$. Notons $j_{v}$ l'inclusion de $L$ dans $v^{-1}\left(M^{\prime}\right)$. Le diagramme suivant commule :

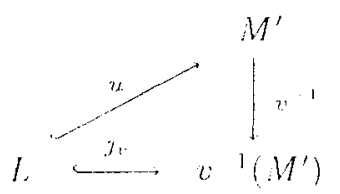


Or, on a que $W^{\prime}=\left(\tilde{f}_{p}\right)_{x,}(M)=v u_{z} v^{\prime}(M)$. Puisque $\iota^{\prime} \in \widetilde{O}(W, M)$, on a que $v^{\prime}(M)=M$. Donc,

$$
M^{\prime}=v u_{i}(M) \text { co qui veut dire que } v^{-1}\left(M^{\prime}\right)=u_{i}(M) .
$$

\section{Remarque :}

Grâce à la proposition 2.39, nous savons que l'indice $\left[O\left(u_{i}(M)\right): O\left(W, u_{i}(M)\right)\right]$ est égal au nombre de représentations de $L$ par $u_{i}(M)$ qui som dans la même classe que l'inclusion $L \longrightarrow u_{i}(M)$.

\section{Définition 2.45}

On sait qu'il est possible de choisir $M=M_{1}, \ldots, M_{k}$ des représentants des classes d'isomorphismes dans le genre de $M$. Soit $s \in I_{k}$. Posoms $a\left(L, M_{s}\right)$, le nombre de reprósentations de $L$ par $M_{s}$ dans le genre de $j: L \longleftrightarrow M$.

\section{Proposition 2.46}

Soit $s \in I_{k} k$ alors on a :

$$
u\left(L, M_{s}\right)=\sum_{i \in I}\left[O\left(u_{i}(M)\right): O\left(W, u_{i}(M)\right)\right]
$$

où $I=\left\{i \in I_{m} \mid u_{i}(M) \simeq M_{s}\right\}$. (I peut être ćventuellement vide.)

\section{Démonstration :}

Soient $i_{1}, \ldots, i_{r}$ tels que $u_{i_{j}}(M) \simeq M_{s} \forall j \in\left[I_{r}\right.$. Pour tout $j \in \mathbb{I}_{r}$, on se fixe un isomorphisme $g_{j}$ de $u_{i_{j}}(M)$ dans $M_{s}$ préservant les formes quadratiques, airsi que $h_{j}: L \longrightarrow M_{s}$ qui est la composition de l'incusion de $L$ dans $u_{i_{j}}(M)$ aves $g_{j}$.

Nous allons tout daberd montrer que l'onsernble des $h_{j}$ constitue un système complet de représentants de representations de $L$ par $H_{i}$ gui sont dans le meme genre que l'inclusion $j: L \hookrightarrow M$.

En effet, soit $u: L \longrightarrow W_{s}$ de meme genre que l'inclusion de $L$ dans $M$. Vu la proposition 2.44, il existe un unique $j \in ! !_{r}$ et $f_{j}: M_{s} \longrightarrow u_{i_{j}}(M)$, tels que $f_{j} u$ soit l'inclusion de $L$ dans $u_{i_{j}}(M)$. Or, par construction, $g_{j}^{-1} h_{j}$ cst alussi égale à l"inchusion de $L$ dans $u_{i_{j}}(M)$. Donce $\left(g_{j} f_{j}\right) u=h_{j}$; ce qui veut dire que $u \simeq h_{j}$.

Soit $j \in H_{r}$. Posons $X_{j}$, l'onsemble des représentations de $L$ par $u_{i_{j}}(M)$ qui sont dans la même classe que l'inclusion $L \smile u_{i_{j}}(M)$, ot $Y_{j}$, l'onsemble des représentations de $L$ par $M_{s}$ qui sont dans la classe de $h_{j}$. Alors, l'application

$$
\begin{aligned}
\vartheta: X_{j} & \longrightarrow Y_{j} \\
u & \longmapsto g_{j} u
\end{aligned}
$$

est une bijection.

Le fait que $\psi$ soit bien délinie et injective se démontre de la même manière que (*). Soit $v \in Y_{j}$; il est clair que $g_{j}^{-1} v$ est darns $X_{j}$; donc $\psi$ est bijective.

Finalement, on a :

$$
a\left(L, M_{*}\right) \stackrel{(\oplus)}{=} \sum_{j}^{r}\left|X_{j}\right|=\sum_{i=1}\left[O \left(u_{i}(M): O\left(W, U_{i}(M)\right]\right.\right.
$$

La deuxième égalité est une conséguence directe de (**) et de la remarque précédant la définition 2.45. 


\section{Théorème 2.47}

On a l'égalité :

$$
\left.m(o(W))^{\widetilde{O}(W)}\right)=\mu(\widetilde{O}(W, M)) \sum_{s=1}^{m} \frac{a\left(L, M_{s}\right)}{\left|O\left(M_{k}\right)\right|}
$$

\section{Démonstration :}

C'est un corollaire imrrédial des propossitions 2.43 el 2.46 .

\section{Définition 2.48}

Cette égalité est appelée formule de Mnkowski-Siegel. Nous nous intéresserons plus particulièrement à un cas particulier de cette formule :

\section{Corollaire 2.49}

Si $L=U=\{0\}$, alors quel que soit $: \in \|_{k}$, il est clair que $a\left(L, M_{s}\right)=1$ ast que $V=W$. De plus, on a vu au paragraphe $D$ que $\prod_{p \in} \mu_{p}$ forme une mesure $\partial(V)$. On obtient alors :

$$
\sum_{s=1}^{m} \frac{1}{\left|O\left(M_{s}\right)\right|}=m\left(\left.\sigma(v)\right|^{\sigma(v)}\right) \prod_{p \in \mathbb{R}} \mu_{p}\left(O\left(M_{p}\right)\right)^{1}
$$

Cette égalité n'ost vraic que si $\prod_{\psi c} \mu_{p}$ converge. Ie paragraphe suivant sera consacré à fixcr les $\mu_{p}$ pour tout $p$ de telle marière: que ce produit converge.

\section{H. Normalisation des $\mu_{p}$.}

\section{Lemme 2.50}

Soient $p \in$ et $V_{p}=V \Leftrightarrow$, où $V$ ut notre -espace vectoriel bilinéaire ou quadratique, de dimension

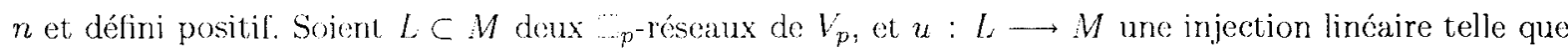
$L^{*}=\beta_{q}(u(\cdot), M)$. Supposons qu'il existe $k \in I$ tel que $p^{k \cdot 1} q(M) \subset \cdots p$ et wel que $q(u(x)) \equiv q(x) \bmod p^{k}$. Alors, il existe $u^{\prime}: L \longrightarrow V_{p}$ linéaire, tolle que $u^{\prime}(x) \equiv u(x) \bmod p^{k} M$ pour tout $x \in L$, ot $q\left(u^{\prime}(x)\right) \equiv$ $q(x) \bmod p^{k \cdots 1}$.

\section{Démonstration :}

Soic $f: L \longrightarrow M$ à détominer, linéaire, telle que $u^{\prime}(x)=u(x)+p^{k} f(x)$. D'autre part, il existe $g: L \longrightarrow$ $\rightarrow p$ telle que $q(u(x))=g(x)+g(x) p^{k}$. Los égalites suivantes sont satisfaites :

$$
\begin{aligned}
\beta_{\eta}(x, y)+q(x)+q(u)+g(x+y) p^{k} & =q(x+y)+q(x+y) p^{k}=q(u(x+y))=q(u(x)+u(y)) \\
& =\beta_{q}(u(x), u(y))+q(u(x))+q(u(y)) \\
& =\beta_{q}(u(x), u(y))+q(x)+g(x) p^{k}+q(y)+g(y) p^{k} .
\end{aligned}
$$

Donc,

$$
g(x+y)-g(x)-g(y)=p^{k}\left(\beta_{q}(u(x), u(y))-\beta_{q}(x, y)\right) \in \cdots, \quad \forall x, y \in L
$$

Il existe une forme bilinéaire $: \quad: \times l \rightarrow \ldots$, non forcément symétrique, telle que $\gamma(x, x)=g(x) \forall x \in L$. Calculons :

$$
\begin{aligned}
q\left(u^{\prime}(x)\right) & =q\left(u(x)+p^{k} f(x)\right)=\beta_{q}(u(x), f(x))+q(u(x))+p^{2 k} q(f(x)) \\
& =p^{k} \beta_{q}(u(x), f(x))+q(x)+p^{k} q(x, x)+p^{2 k} q(f(x)) \\
& \equiv q(x)+p^{k}\left(\beta_{q}(u(x), f(x))+\gamma(x, x)\right) \quad\left(\bmod p^{k+1}\right) .
\end{aligned}
$$


Il nous faut done trouver $\int$ telle que

$$
\beta_{q}(u(x), f(x)) \equiv-\gamma(x, x) \quad(\bmod p) .
$$

Fixons $y_{0} \in J$. La forme $-\gamma\left(x, y_{0}\right)$ est un élément de $L^{*}$. Par hypothèse, il existe $f\left(y_{0}\right) \in M$ tel que $-\gamma\left(x, y_{0}\right) \equiv \beta_{q}\left(u(x), f\left(y_{0}\right)\right) \bmod p$. Soient $y_{0}, y_{1} \in L$, on a :

$$
\beta_{q}\left(u(x), f\left(y_{0}+y_{1}\right)\right)=-\gamma\left(x, y_{0}+y_{1}\right)=-\left(\gamma\left(x, y_{0}\right)+\gamma\left(x, y_{1}\right)\right)=-\left(\beta_{q}\left(u(x), f\left(y_{0}\right)\right)+\beta_{q}\left(u(x), f\left(y_{1}\right)\right)\right) .
$$

Ce qui veut dire que

$$
\beta_{q}\left(u(x), f\left(y_{0}+y_{1}\right)-f\left(y_{0}\right)-f\left(y_{1}\right)\right)=0
$$

ceci, quel que soit $x$. Or, $u$ est injuctive, done son image contient un base de $V_{p}$. De plus $\beta_{q}$ est non dégénérée. Donc $\int$ est linćaire.

Nous allons déterminer au lemme suivant le nombre d'applications $\int$ modulo $p M$ que nous cherchions lors de la démonstation du lemme précédent, donc le nombre possible de $u^{\prime}$ modulo $p^{k+1} M$ satisfaisant la conclusion de co lemme.

\section{Lemme 2.51}

Dans les mêmes hypothìses que pour le lemme précédent; on a exactement $p^{\frac{n(n-1)}{2}}$ possibilités pour $f$ modulo $p M$.

\section{Démonstration :}

Soit $\|_{p}=Z_{p} / p_{p}$. $L$ et $M$ peuvent etre vus comme des ${ }_{p}$-espaces vectoriel. Posons $\mathscr{L}_{\mathbb{W}_{p}}(L, M)$ l'espace des applications linéaires de $L$ dans $M$, et $\mathscr{C}_{h} \mathscr{A}_{p}\left(L, \eta_{p}\right)$ celui des formes bilinéaires symétriques de $L \times L$ dans $\mathbb{F}_{p}$. L'application

$$
\begin{aligned}
& 0: \mathscr{Q}_{n}(L, M) \longrightarrow \mathscr{W}_{n}\left(L, \mathbb{W}_{p}\right) \\
& g \longmapsto B_{q}(u(\cdot), g(\cdot))
\end{aligned}
$$

est clairement linćaire et surjective, car $\beta_{\text {q }}$ st non dégénćrée ot $u$ cst injective. Par le théorème du rang, on a que la dimersion du noxiau de: a vaut $n^{2}-\frac{n(n \cdot 1)}{2}=\frac{n(n \cdot 1)}{2}$. Donce, il existe $p^{\frac{n(n-1)}{2}}$ éléments de $\mathscr{L}_{\mathbb{H}_{p}}(L, M)$ qui vom sur $\mathrm{k}-\gamma(x, x)$ du lemme précédent.

\section{Lemme 2.52}

Soient $L, M$ dans $V_{p}$, et $u$ comme au lemme 2.50. Alors il existe $\bar{u}: L \longrightarrow V_{p}$ linéaire, telle que $\bar{u}(x) \equiv u(x) \bmod p^{k} M$ at telle que $q(\bar{u}(x))=q(x)$ quel que soil $x$ dans $L$.

\section{Démonstration :}

Par le lemme 2.50, il cxiste $u^{\prime}$ tel que $u^{\prime}(x) \equiv u(x) \bmod p^{k} M$ et $q\left(u^{\prime}(x)\right) \equiv q(x) \bmod p^{k-1}$. Or, on a que $\beta_{q}\left(u^{\prime}(\cdot), M\right)=\beta_{q}(u(\cdot), M)+\mu^{k} \beta_{q}(J(\cdot), M)=I^{*}$. Donc, $u^{\prime}$ satisfait les conditions du lemme 2.50 mais pour $k+1$ cente foris-ci. 11 existe alors $u^{\prime \prime}$ tel que:

$$
\begin{aligned}
u^{\prime \prime}(x) & \equiv u^{\prime}(x) \quad\left(\bmod p^{k+1} M\right) \\
q\left(u^{\prime \prime}(x)\right) & \equiv q(x) \quad\left(\bmod p^{k+2}\right) .
\end{aligned}
$$

Par induction, il est done possible de se définir pour tout $n \in\left[\right.$ I, une application linéaire $u^{(n)}: L \longrightarrow V_{p}$ telle que :

$$
\begin{aligned}
u^{(n)}(x) & \equiv u^{(n-1)}(x) \quad\left(\bmod p^{k+n-1} M\right) \\
q\left(u^{(n)}(x)\right) & \equiv \eta(x) \quad\left(\bmod p^{k+n}\right) .
\end{aligned}
$$

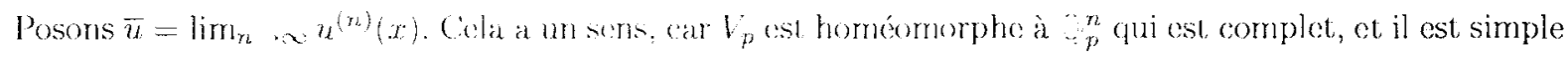
de voir que la suite $\left(u^{(n)}\right)$ ne ce cal de Cauchy. On a dome que $\bar{u}$ est linéaire. De plus $q(\bar{u}(x))=q(x) \forall x$, car la suite $q\left(u^{(n)}(x)\right)-q(x)$ comverge vers 0 dans $\ldots .$. 


\section{Proposition 2.53}

Soient $M$ un ‥-róscan cuadraticque do $V: p \in$, et $k \in 1 !$ tel que $p^{k} M_{p}^{\#} \subset M_{p}$ et tel que $p^{k-1} q\left(M_{p}^{\#}\right) \subset \mathbb{Z}_{p}$. Alors

Où $\mu_{p}$ est une mesure sur $O\left(V_{p}\right)$.

$$
\frac{\mu_{p}\left(O\left(V_{p}, M_{p} / p^{k} \cdot M_{p}^{\#}\right)\right)}{\mu_{p}\left(O\left(V_{p}, M_{p} / p^{k+1} M_{p}^{\#}\right)\right)}=p^{\frac{n(n-1)}{2}}
$$

\section{Démonstration :}

Par propriété de $\mu_{p}$, il suflit de montrer que l'indice $\left[O\left(V_{p}, M_{p} / p^{k} M_{p}^{\# l}\right): O\left(V_{p}, M_{p} / p^{k+1} M_{p}^{\# \#)}\right)\right.$ vaut $p^{\frac{n(n-1)}{2}}$.

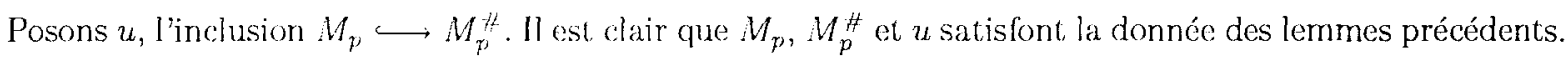
L'ensemble des applications $\bar{u}$ trouvées lors du Jemme 2.52 n'est autre que $O\left(V_{p}, M_{p} / p^{k} M_{p}^{\#}\right)$. Le lemme 2.51 nous permet de conclure sachant que les $u^{\prime}$ du lemme 2.50 sont congrus aux $\bar{u}$ modulo $p^{k+1} M$.

\section{Lemme 2.54}

Soient $L$ et $M$ deux ${ }_{n}$-résceun de $V_{p}$ thels que $[M: L]=p$. Alors on peut trouver $e_{1}, \ldots, e_{n} \in V_{p}$ tels que

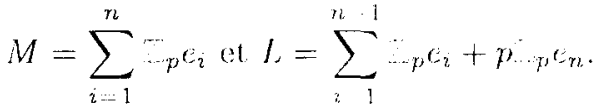

\section{Démonstration :}

On a que $M / L$ est un groupe additif à $p$ éléments, il est done cngendré par un élément $\bar{x}$. Soit $x$ un représentant de $\bar{x}$. Par choix de $x, p x$ cest dans 1 , mais pas $x$ lui-mône; $p x$ est donc primitif. Il existe alors $e_{1}, \ldots, c_{n-1}$ tels que $\left(\epsilon_{1}, \ldots, c_{n}, 1, p x\right)$ soil une base de $L$. Il est clair que $\left(e_{1}, \ldots, e_{n}, 1, x\right)$ engendre $M, \operatorname{car} M=\bigsqcup_{i=1}^{p} i x+1$

\section{Lernme 2.55}

Soient $L$ et $M$ deux $\ldots$-réseaux de $V_{p}$ tels que $[M: L]=p$. Alors $\left[L^{\#}: M^{\# t}\right]=p$.

\section{Démonstration :}

Prenons $e_{1}, \ldots, e_{n}$ tels que $M=\sum_{i=1}^{n} \ldots, c_{i}$ et $L=\sum_{i \cdots 1}^{n-1} \cdots e_{i}+p_{\ldots p} c_{n}$. Soient $c_{1}, \ldots, c_{n}$, définis lors de la proposition 1.21, tels que $M^{H}=\sum_{i}^{n} \cdots p c_{i}$, On a :

$$
I^{\prime \prime}=\sum_{i=1}^{n+1} \ldots c_{i}+\ldots p c_{n}^{\prime}
$$

Et, en se souvenant de la définition des $c_{i}$, on voit facilement que $c_{n}^{\prime}=p^{1} c_{n}$.

\section{Proposition 2.56}

Soient $L, M$, deux ${ }_{p}$-réseaux de $v_{p}$ tels que $[M: I]=p$, et $k \in l 1$ tel que $p^{k-1} M^{H} \subset M, p^{k-1} L^{\#} \subset L$ ct $2 k-4 \geq k$, alors:

$$
\left[O\left(V_{p}, L / p^{k} L / H\right): O\left(V_{p}, M / p^{k} M^{\prime \prime}\right)\right]=p^{n-1}
$$

\section{Démonstration :}

Choisissons-nous $c_{1}, \ldots, c_{n}$ tels que $M=\sum_{i=1}^{n} \cdots p e_{2}, L=\sum_{i=1}^{n \cdots} 1 \cdots e_{i}+p_{-p} e_{n}, M^{H}=\sum_{i=1}^{n} \mathbb{Z}_{p} c_{i}$ et $L^{\#}=\sum_{i=1}^{n \cdots 1} \cdots_{p} c_{i}+p^{1 \cdots} c_{p} c_{n}$.

Soit $u \in O\left(V_{p}, L / p^{k} L^{\#)}\right)$. Pour tout $i \in[]_{n-1}$, on a :

$$
u\left(c_{i}\right)=e_{i}+x_{i} \quad \operatorname{avec} x_{i} \in p^{k} L^{\not z} .
$$

Puisque $\left[p^{k} L^{* t}: p^{k} M^{H t}\right]=p$, le nombre des images $u\left(e_{2}\right)$ modulo $p^{k} M^{\# t}$ possible est an plus de $p$ pour chaque $i \in !_{n-1}$ : done on tout $\gamma^{n}$ ! 


\section{Lernme}

Soient $u$ et $u^{\prime} \in O\left(V_{p}, L / p^{k} L_{i}^{\#}\right)$. Si

$$
\begin{aligned}
u\left(c_{i}\right) & =c_{i}+x_{i}, x_{i} \in p^{k} L^{\prime \prime} \\
\text { et. } \quad u^{\prime}\left(c_{i}\right) & =c_{i}+x_{i}^{\prime}, x_{i}^{\prime} \in p^{k} L^{H} \forall i \in !_{n-1}
\end{aligned}
$$

sont tels que $x_{i} \equiv x_{i}^{\prime} \operatorname{mond} p^{k} M^{\prime \prime}$. Alors, $u \equiv u^{\prime} \bmod O\left(V_{p}, M / p^{k} M^{\# t}\right)$.

Ce lemme implique que l'indice cherché est inléricur ou égal à $p^{n \cdots 1}$.

\section{Démonstration du lemme :}

Puisque $x_{i} \equiv x_{i}^{\prime} \bmod p^{k} M^{\#}$, il est clair que $u^{\prime}\left(e_{i}\right) \equiv u\left(e_{i}\right) \bmod p^{k} M^{\#}$, done que $\left(u^{-1} u^{\prime}\right)\left(e_{i}\right) \equiv e_{i} \bmod$

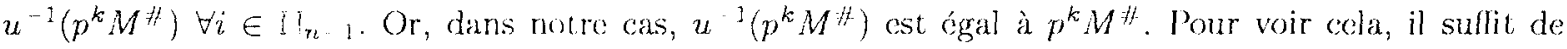
montrer que $\beta_{q}\left(u\left(c_{i}\right), c_{3}\right) \in \cdots, \quad \forall i, j \in I I_{n}$.

1) Si $j \neq n, e_{j} \in L$, et $c_{i} \in L^{\prime \prime}$ quel que soil $i$; alors, $\beta_{q}\left(u\left(c_{i}\right), e_{j}\right) \in \cdots p$.

2) Si $i=j=n$, on a que $p^{-1} c_{n} \in L^{\text {th }}$ et $p c_{n} \in L$. Donc, $\beta_{q}\left(u\left(c_{n}\right), e_{n}\right)=\beta_{q}\left(u\left(p^{-1} c_{n}\right), p e_{n}\right) \in w_{p}$.

3) Si $i \neq n=j$, vu la définition des $c_{i}$, il ess. clair que $\beta_{q}\left(u\left(c_{i}\right), u\left(e_{n}\right)\right)=\beta_{q}\left(c_{i}, e_{n}\right)=0$. Donc, $\beta_{q}\left(u\left(c_{i}\right), e_{n}\right)=$ $\beta_{q}\left(u\left(c_{i}\right), c_{n}-u\left(c_{n}\right)\right)$. Or, $p_{n}-u\left(p c_{n}\right) \in p^{k} L^{H}$, co qui veut dire que $c_{n}-u\left(c_{n}\right) \in p^{k-1} L^{\# t} \subset L$. On a alors $\beta_{q}\left(u\left(c_{i}\right), \epsilon_{n}\right) \in \cdots, p$ puisque $u\left(c_{i}\right) \in L^{H}$.

Il reste à montrer que $\left(u^{\prime} u^{\prime}\right)\left(e_{n}\right):=\bar{u}\left(e_{n}\right) \equiv c_{n} \operatorname{mrod} p^{k} M^{\#}$.

On sait que $\bar{u}\left(e_{n}\right)-c_{n} \in M^{H}$, donc, $\bar{u}\left(c_{n}\right)-c_{n}=\sum_{i=1}^{n} l_{i} c_{i}$ avec $l_{i} \in \ldots p$. Il reste à voir que $t_{i} \in p^{k Z_{p}}$. Or, $p e_{n} \in L$, donc $\bar{u}\left(p c_{n}\right)-q m_{n} \in p^{k} L^{\prime \prime}$. On a alors $\sum_{i=1}^{n} p l_{i} c_{i} \in p^{k} L^{\prime H}$. Cola veut dire que $\iota_{n} \in p^{k}{ }^{2} \mathbb{Z}_{p}$ et que $t_{i} \in p^{k} 1_{p} \ldots$

Dautre part, on a que $B_{\eta}\left(\bar{u}\left(c_{2}\right), \bar{u}\left(c_{n}\right)\right)=\xi_{q}\left(c_{2}, c_{n}\right)$ pour tout $i \in ! !_{n}$. Il est facile de voir que $\bar{u}\left(e_{2}\right)=$ $e_{i}+u^{-1}\left(x_{i}^{\prime}-x_{i}\right) \forall i \in !_{n}$ 1: donce. $\bar{u}\left(c_{i}\right)-c_{n} \in p^{k} M^{\prime \prime}$. 1) o plus, $\bar{u}\left(e_{n}\right)-e_{n} \in L \subset M$; alors, $\bar{u}\left(e_{n}\right) \in M$. Finalement, on obticnt:

$$
\beta_{q}\left(c_{i}, c_{n}\right)=\beta_{q}\left(\bar{u}\left(c_{i}\right), \bar{u}\left(\epsilon_{n}\right)\right) \equiv \beta_{q}\left(c_{i}, \bar{u}\left(\epsilon_{n}\right)\right)=\beta_{q}\left(c_{i}, e_{n}\right)+t_{i} \quad\left(\bmod p^{k}\right) \quad \forall i \in ! 1_{n-1} .
$$

Donc, $t_{i} \in p^{k \ldots}, \forall i \in I I_{n} \quad$.

Occupons-rous maintenant de $t_{n}$, on a :

$$
\begin{aligned}
q\left(u\left(c_{n}\right)\right) & =q\left(c_{n}+\sum_{i=1}^{n} t_{i} c_{i}\right)=q\left(c_{n}\right)+q\left(\sum_{i=1}^{n} t_{i} c_{i}\right)+\beta_{q}\left(c_{n} ; \sum_{i=1}^{n} t_{i} c_{i}\right) \\
& =q\left(c_{n}\right)+q\left(\sum_{i=1}^{n} t_{i} c_{i}\right)+t_{n} \equiv q\left(c_{n}\right)+t_{n} \quad\left(\bmod p^{k \cdots \cdots p}\right) .
\end{aligned}
$$

La dernière équivalence vient du fait que thacun des $t_{i}$ est au moins divisible par $k-2$, et parce que $2 k-4 \geq k$. Done $t_{n} \in p^{k \ldots . . .}$. Ainsi siacheve la démonstration du lemme.

Terminons la preuve de notre proposition:

L'indice $[M: p M]$ raut $p^{n}$. In multipliant sucessivement par $p$ chaque élóment de la base de $M$, il est possible de trouver une chaine de réscalux $L_{0}=M \supset L_{1} \supset \cdots \supset L_{n}=p M$ tols que $\left[I_{t_{i}}: L_{i+1}\right]=p$. Par le lemme, on as que:

$$
\left[O\left(V_{p}, L_{2} / p^{k} I_{i}^{\| \prime}\right): O\left(V_{p}, L_{i}, l_{p}^{k} L_{i=1}^{\| t}\right)\right] \leq p^{n} \quad 1 \quad \forall i
$$

Grâce à la multiplicantivilé dese indices, on trouve:

$$
\left[O\left(V_{p}, p: M / p^{k}(p: M)^{\#)}\right): O\left(V_{p}, M / p^{k} M^{\#)}\right)\right] \leq p^{n(n+1)} .
$$

Or, $O\left(V_{p}, p M / p^{k}(p M)^{\prime \prime}\right)=O\left(V_{p}, M / p^{k \cdot 2} M^{H}\right)$, et la proposition 2.53 nous dit que

$$
\left[O\left(V_{p}, M / p^{k-2} M^{\sharp k}\right): O\left(V_{p}, M / p^{k} M^{\sharp \hbar}\right)\right]=p^{n(n-1)} .
$$

On peut conclure, de nouveau grâce à lia multiplicativilé des indices. 


\section{Définition 2.57}

Soient $M$ un réseau quadratique de $V, p \in \mathbb{R}, k$ tel que $p^{k} M_{p}^{\text {;f }} \subset M_{p}$ et $\mu_{p}$ une mesure sur $O\left(V_{p}\right)$. Posons

$$
c_{\mu, p}=\mu_{p}\left(O\left(V_{p}, M_{p} / p^{k} M_{p}^{\# t}\right)\right) \cdot\left[M_{p}: p^{k} M_{p}^{\# t}\right]^{\frac{n-1}{2}} .
$$

On pourrait se demander pourguoi $c_{\mu, p}$ ne s'appelle pas plutôt $c_{\mu, p, k, M}$. Nous allons voir dans le thèorème prochain que si $k$ cst asse $\%$ grand, alors $c_{\mu, p}$ est constant, et que de plus il est indépendant du réseau quadratique $M$ choisi.

\section{Théorème 2.58}

Si $k$ est tel que $p^{k-1} q\left(M_{p}^{\# k}\right) \subset \cdots_{p}$ et $p^{k} M_{p}^{\#} \subset M_{p}$, alors $c_{\mu, p}$ est constant quand $k$ croît, il est en outre indépendant du réseau quadratique choisi.

\section{Démonstration :}

On a vu à la propmsition 2.53 que?

$$
\frac{\mu_{p}\left(O\left(V_{p}, M_{p} / p^{k} M_{p}^{\#}\right)\right)}{\mu_{p}\left(O\left(V_{p}, M_{p} / \gamma^{k+1} \cdot M_{p}^{\# k}\right)\right)}=p^{\frac{n(n, n)}{2}}
$$

De plus, on a :

$$
\left[M_{p}: p^{k+1} M_{p}^{\# t}\right]^{\frac{n+1}{2}}=\left[M_{p}: p^{k} \cdot M_{p}^{\# /}\right]^{\frac{n+1}{2}} \cdot\left[p^{k} M_{p}^{\# /}: p^{k+1} M_{p}^{\# 1}\right]^{\frac{n+1}{2}}=\left[M_{p}: p^{k} M_{p}\right]^{\frac{n-1}{2}} \cdot p^{\frac{n(n-1)}{2}} .
$$

Donc, $c_{\mu, p, k, M}=c_{\mu, p, k \cdot 1, M}$.

Soient $L$ et $M$ deux ‥-réseaux quadratiques de, $V$ tels que $L_{p} \subset M_{p}$. Un bref raisonnement nous permet de dire qu'il existe $s \in\left[1\right.$ lel que $\left[M_{p}: L_{p}\right]=p^{s}$. I] sulfit done de montrer que $c_{\mu, p, M}=c_{\mu, p, L}$ avec $\left[M_{p}: L_{p}\right]=p$. Puisque nous verons de voir que notre $c_{\mu, p}$ était constant si $k$ croît, on peut supposer $k$ assez grand de telle manière que les hypothèses de la proposition 2.56 soient satisfaites. il s'ensuit alors que

$$
\frac{\mu_{p}\left(O\left(V_{p}, L_{k} / p^{k} L_{p}^{\#}\right)\right.}{\mu_{p}\left(O\left(V_{p}, M_{p} / p^{k} M_{p}^{\# f}\right)\right.}=p^{n(n-1)}
$$

Mais, on a les ćgalitós suivantes:

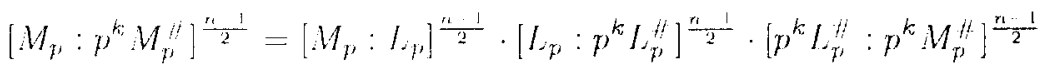

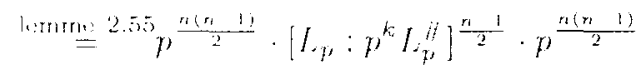

$$
\begin{aligned}
& =p^{n(n+1)} \cdot\left[L_{p}: p^{k} L_{p}^{i t}\right]^{\frac{n-1}{2}} \text {. }
\end{aligned}
$$

Donc, $c_{\mu, p, M}=c_{\mu, p, l}$.

Nous sommes près maintenant à normaliser nos $\mu_{p}$. Rappelons qu'il faut fixer les mesures $\mu_{p}$ de telle sorte que

$$
\prod_{p \in} \mu_{p}\left(O\left(M_{p}\right)\right)^{-1}
$$

converge. Il est clair que $M_{p}=M_{p}^{\prime \prime}$ pour presque tout $p$, il suffit done de voir que le produit converge pour de tels $p$. Pour cos $p$, on peut choisir $k=1$ dans le théorème précédent. Personne ne peut m'empêcher de dire que :

$$
\mu_{p}\left(O\left(M_{p}\right)\right)=\left[O\left(M_{p}\right): O\left(V_{p}, M_{p} / p \cdot M_{p}^{\#}\right)\right] \cdot \mu_{p}\left(O\left(V_{p}, M_{p} / p M_{p}^{\#}\right)\right) .
$$

On trouve alors, grâce au théorème précédent que

$$
\mu_{p}\left(O\left(V_{p}, M_{p} / p M_{p}^{\prime \prime}\right)\right)=c_{\mu, p} \cdot\left[M_{p}: p M_{p}\right]^{\frac{1-n}{2}}=c_{\mu, p} \cdot p^{\frac{n(1) n)}{2}} .
$$


Dautre part, posons $\bar{M}_{p}$ qui est. $M_{p} / p M_{p}$ vu comme ${ }^{-e s p a c e ~ v e c t o r i e l, ~ a ~ p o s o n s ~ a u s s i ~} O\left(\overline{M_{p}}\right)$ le groupe orthogonal pour la forme héritic de colle de $V_{p}$. Si $p \neq 2$, il cst clair que le groupe orthogonal quadratique coücide avec Io groupe orthegonal bilinciare. Si $p=2$ est un "bon premicr", on pose $O\left(\overline{M_{2}}\right)$ comme étant le groupe orthogenal quadralicule sur $\bar{W}_{2}$, car notre formo quadratique n'ost pas issue d'une forme bilinéaire. En effet puisque $M_{2}=M_{2}$ a que lon a loujours que $\left[M_{2}^{\| t}: M_{2}\right]=$ det $\beta_{q}$, on en déduit que $\beta_{q}$ est non dégénéré sur $M_{2}$, ce qui noest jumais le cas si q provient d'une forme bilinéaire. Alors, on a:

$$
\left[O\left(M_{p}\right): O\left(V_{p}, M_{p} / p M_{p}\right)\right]=\left|O\left(\overline{M_{p}}\right)\right|
$$

En efret, l'application

$$
\begin{aligned}
\psi: O\left(M_{p}\right) & \longrightarrow O\left(\overline{M_{p}}\right) \\
u \longmapsto & \bar{u}: \overline{M_{p}} \longrightarrow \overline{M_{p}} \\
& x_{p}+p M_{p} \longmapsto u\left(x_{p}\right)+p M_{p} .
\end{aligned}
$$

est clairement bien définie, son noyau est ()$\left(V_{p}, M_{p} / p M_{p}\right)$, et elle est surjective, on vertu du lemme 2.52 . En résumé, on a :

$$
\mu_{p}\left(O\left(M_{p}\right)=c_{\mu, p} \cdot p^{\frac{n(1, n)}{2}} \cdot\left|O\left(\overline{M_{p}}\right)\right|\right.
$$

Pour de tels $p$, on verra all chapitre suivant que

$$
\left|O\left(\overline{M_{p}}\right)\right|=2 \cdot p^{\frac{n(n, 1)}{2}} \cdot \prod_{0<2,2<n}\left(1-p^{22}\right) \cdot \begin{cases}1 & \text { si } n \text { est impair } \\ \left(1-\left(\frac{d}{p}\right) \cdot p^{\frac{n}{2}}\right) & \text { si } n \text { est pair }\end{cases}
$$

Où $\left(\frac{\cdot}{p}\right)$ est le symbole de locgendre, ot où $d$ est le discriminant de $M$.

Finalement, on a :

\section{Théoròne 2.59}

Dans les mêmes hypotheses que prócédemment, on a :

$$
\mu_{p}\left(O\left(M_{p}\right)\right)=2 \cdot c_{\mu, p} \cdot \prod_{0<2 i<r_{2}}\left(1-p^{22}\right) \cdot \begin{cases}1 & \text { si } n \text { est impair } \\ \left(1-\left(\frac{d}{p}\right) \cdot p^{\frac{n}{2}}\right) & \text { si } n \text { est pair }\end{cases}
$$

\section{Démonstration :}

C'est immédiat.

Ce produit converge pour tout $n \geq 2$ si on lise pour tout $p, \mu_{p}\left(O\left(V_{p}, M_{p} / p^{k} M_{p}^{\text {\#k }}\right)\right)$ de telle manière que

$$
c_{\mu, \psi, p}=\frac{1}{2}
$$

Ainsi, nous avons pu nommaliser ros mesures. 


\section{CHAPITRE 3}

\section{Le groupe orthogonal sur les corps $\mathbb{F}_{p}$}

Dans ce chapitre, nous allons calculer le cardinal du groupe orthogonal pour certaines formes quadratiques non dégénérées sur $\mathbb{F}_{p}, p$ premier. Tout d'abord, nous allons "classifier" grossièrement les formes sur $\mathbb{F}_{2}$, puis sur $\mathbb{F}_{p}$ avec $p$ premier impair, pour finalement calculer le cardinal de $O_{q}^{n}\left(\mathbb{F}_{p}\right)$ cas par cas.

\section{A. Formes quadratiques non dégénérées sur $\mathbb{F}_{2}$.}

Fixons-nous $(V, q)$ un espace quadratique non dégénéré sur $\mathbb{F}_{2}$. On sait que $\beta_{q}(x, x)=2 q(x)=0 \forall x \in V$. Donc $\beta_{q}$ est une forme "alternéc", de déterminant 1.

\section{Proposition 3.1}

$$
\beta_{q} \simeq<\left(\begin{array}{ll}
0 & 1 \\
1 & 0
\end{array}\right)>\boxplus \cdots \boxplus<\left(\begin{array}{ll}
0 & 1 \\
1 & 0
\end{array}\right)>
$$

\section{Démonstration :}

Soient $x, y \in V$ tels que $\beta_{q}(x, y)=1$. Do tels vecteurs existent puisque $q$ est non dégénérée.

On a donc $\left(V, \beta_{q}\right) \simeq\langle x, y\rangle \boxplus\langle x, y\rangle^{\circ}=\left\langle\left(\begin{array}{ll}0 & 1 \\ 1 & 0\end{array}\right)\right\rangle \boxplus A$, où la matrice $A$ est du même type que $M_{\beta_{q}}$, car on vérifie aisément que $\int_{\beta \mid}$ est. un isomorphisme. Donc en faisant le mêrne raisonnement sur $\left(\langle x, y\rangle^{\perp},\left.\beta\right|_{<x, y\rangle^{\perp}}\right)$, on conclut.

\section{Remarque :}

On obtient donc que la dimension de $V$ est paire. On aurait tout de même envie de dire que certaines formes sont "moins dégénérées que d'autres" si le rang de $\beta_{q}$ est $n-1$ par exemple. On pourrait se donner de nouvelles définitions, par exemple de semi-régularités ou de défectivités. De tels raffinements sont faits dans la littérature, par exemple dans [6, ch. $I, \S 16]$ ct dans $[7, \mathrm{pp} 6-7]$. On pourrait alors avoir une classification plus finc, mais cela alourdirait mon cxposć, surtout que nous n'en n'aurons pas besoin pour la suite des événements.

\section{Corollaire 3.2}

$V \simeq H \boxplus \cdots \boxplus H$ ou $V \simeq H \boxplus \cdots \boxplus H \boxplus L$.

Oid $(H, \mu)$ est la forme hyperbolique de dimension $2: \mu\left(\left(t_{1}, t_{2}\right)\right)=t_{1} \cdot t_{2}$ et $(L, \nu)$ est la forme "bihyperbolique" de dimension $2: \nu\left(\left(t_{1}, t_{2}\right)\right)=t_{1}^{2}+t_{1} \cdot t_{2}+t_{2}^{2}$.

\section{Démonstration :}

la proposition 3.1 nous dit que $(V, q) \simeq\left\langle x_{1}, y_{i}\right\rangle \boxplus \cdots \boxplus\left\langle x_{\frac{n}{2}}, y_{\frac{n}{2}}\right\rangle$ avec $q\left(x_{i}\right)=a_{i}$ et $q\left(y_{i}\right)=b_{1}$ pour tout $i \in I_{\frac{n}{2}}$.

Or, si $\left(a_{i}, b_{i}\right)=(0,0)$ alors $\left.q\right|_{\left\langle x_{i}, y_{i}\right\rangle}=\mu$, si $\left(a_{i}, b_{i}\right)=(1,1)$ alors $\left.q\right|_{\left\langle x_{i}, y_{i}\right\rangle}=\nu$ et si $\left(a_{i}, b_{i}\right)=(0,1)$ on remplace $y_{i}$ par $y_{i}^{\prime}=y_{i}+x_{i}$ et on obtient $\left.q\right|_{\left\langle x_{i}, y_{i}^{\prime}\right\rangle}=\mu$.

De plus $L \boxplus L \simeq H \boxplus H$; en effet, le changement de base est :

$$
\begin{aligned}
& e_{1} \mapsto e_{1}+e_{3} \\
& e_{2} \mapsto e_{1}+e_{3}+e_{4} \\
& e_{3} \mapsto e_{2}+e_{4} \\
& e_{1} \mapsto e_{2}+e_{3}+e_{1}
\end{aligned}
$$




\section{Remarque :}

Ces observations étant faites, il est donc clair que si $n \equiv 0 \bmod 4$ alors $V \simeq H \boxplus \cdots \boxplus H$. Lors du calcul du groupe orthogonal au paragraphe $\mathrm{C}$, nous ne nous occuperons que de co cas-là.

\section{B. Formes quadratiques sur $p$, $p$ impair}

\section{Lemme 3.3}

Soit $(V, q)$ une forme quadratique de dimension supérieure ou égale à 3 . Il existe $x \neq(0, \ldots, 0)$ tel que $q(x)=0$.

\section{Démonstration :}

On sait que $q$ est diagonalisable puisque la caractéristique est différente de 2. Le problème se résume donc à trouver $x, y, z$ non tous nuls tols que $a x^{2}+b y^{2}+c z^{2}=0$. Posons alors $z=1$, il faut donc trouver $x$ et $y$ tels que $a x^{2}+b y^{2}=-c$. la lemme 1.40 permet de conclure.

\section{Théorème 3.4}

Soit $(V, q)$ un espace quadratique de dimension $n$ non dégénéré sur $\mathbb{F}_{p}, p$ impair. Alors $\beta_{q}$ est isomorphe à une des formes suivantes :

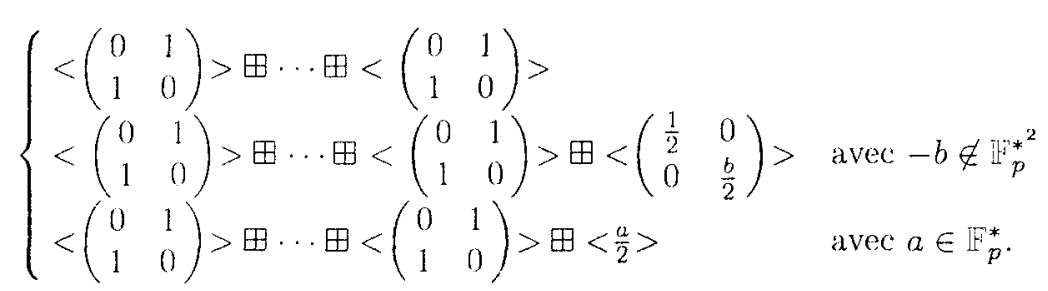

\section{Démonstration :}

Si $n=1$, c'est clair.

Si $n=2$, deux cas se présentent :

a) Il existe $x$ non nul tel que $\beta_{q}(x, x)=0$. Soit $y$ tel que $(x, y)$ soit une base de $V$. Si $\beta_{q}(y, y)=0$, on est content.

Si $\beta_{q}(y, y) \neq 0$, on pose $y^{\prime}=\frac{\beta_{q}(y, y)}{2 \beta_{q}(x, y)} \cdot x+y$. On a $\beta_{q}\left(y^{\prime}, y^{\prime}\right)=0$ et $\beta_{q}\left(x, y^{\prime}\right)=\beta_{q}(x, y)=s \neq 0$. En posant $y^{\prime \prime}=\frac{1}{s} \cdot y^{\prime}$ on en déduit que $\beta_{q} \simeq\left(\begin{array}{ll}0 & 1 \\ 1 & 0\end{array}\right)$.

b) En revanche, si $\beta_{q}(x, x) \neq 0$ pour tout $x \in V \backslash\{0\}$, nous savons grâce au lemme 1.40 qu'il existe $y$ tel que $\beta_{q}(y, y)=\frac{1}{2}$, donc $\beta_{q} \simeq<\frac{1}{2}>\boxplus<\frac{b}{2}>$ avec $-b \notin \mathbb{F}_{p}^{*^{2}}$, car sinon $\beta_{q}$ représenterait 0 .

Finalement, si $n \geq 3$, grâce au lemme 3.4, nous savons qu'il est possible de représenter 0 non trivialement, donc la forme s'écrit $\left(\begin{array}{ll}0 & 1 \\ 1 & 0\end{array}\right) \boxplus F$ avec $F$ de dimension $n-2$. On termine par récurrence.

\section{Corollaire 3.5}

Tout espace quadratique non dégénéré sur $\$ p, p$ impair est isomorphe à l'un des espaces suivants :

$$
\begin{cases}H \boxplus \cdots \boxplus H & \text { Type (A) } \\ H \boxplus \cdots \boxplus H \boxplus J & \text { Type (B) } \\ H \boxplus \cdots \boxplus H \boxplus I & \text { Type (C) }\end{cases}
$$

où $H$ est l'espace hyperbolique, $J$ est l'espace de dimension 2 muni de la forme $j$ définie par $j\left(\left(t_{1}, t_{2}\right)\right)=$ $t_{1}^{2}+b t_{2}^{2},-b \notin \mathbb{F}_{p}^{*^{2}}$, et $l$ est l'espace de dimension 1 muni de la forme $i$ définie par $i(l)=a t^{2}, a \in \mathbb{F}_{p}^{*}$ 


\section{Démonstration :}

Sachant que 2 est inversible, loute forme quadratique est détcrminée entiòrement par sa forme bilinéaire associée $\beta_{q}$.

\section{Le cardinal du groupe orthogonal.}

\section{Lemme 3.6 (théorème de Witt)}

Soient $(V, q),\left(V^{\prime}, q^{\prime}\right)$ et $(W, r)$ trois espaces quadratiques non dégénérés sur $\mathbb{F}_{p}, p$ premier quelconque. Alors :

$V \boxplus W \simeq V^{\prime} \boxplus W$ implique $V \simeq V^{\prime}$.

\section{Remarque :}

Le théorème de Witt n'est pas toujours vraj si l'on considère des espaces bilinéaires, mais cette version est vraie sans conditions supplémentaires sur $W$, même sur des anneaux semi-locaux (donc en particulier sur $\left.\mathbb{F}_{2}\right)$. La démonstration est donnée dans $[2$, ch. III, §4].

\section{Lemme 3.7}

Soit $(V, q)$ un espace quadratique non dégénéré sur $: "$. Posons $\alpha(V)$ le nombre de vecteurs $x$ non nuls tels que $q(x)=0$. Alors or a :

$$
\alpha(V)= \begin{cases}\left(p^{m}-1\right)\left(p^{m}+1\right) & \text { si } V \text { ust du lype }(\mathrm{A}) \text { de rang } 2 m \text { et } p \text { premier quelconque } \\ \left(p^{m}+1\right)\left(p^{m-1}-1\right) & \text { si } V \text { est du type (B) de rang } 2 m \text { et } p \text { premier impair } \\ p^{2 m}-1 & \text { si } V \text { est du type (C) de rang } 2 m+1 \text { et } p \text { premier impair }\end{cases}
$$

\section{Démonstration :}

Par convention, nous écrirons $m \cdot H$ pour $\underbrace{H \boxplus \cdots \boxplus H}_{m \text { fois }}$.

On va chercher un lien entre $\alpha(V)$ et $\alpha(V \boxplus H)$.

Soit $x \in V \boxplus H, x=v+h$ où $v \in V$ et $h=\left(h_{1}, h_{2}\right) \in H$. On a bicn sûr que $q(x)=q(v)+h_{1} \cdot h_{2}$, donc si on cherche à trouver les solutions de $q(v)+h_{1} \cdot h_{2}=0$, on obtient :

$$
\alpha(V \boxplus H)=2(p-1)+\alpha(V)(2 p-1)+\left(p^{n}-\alpha(V)-1\right)(p-1) \quad n=\text { dimension de } V ;
$$

le premier membre comple le nombre do solutions avec $y=0$, le second avec $q(y)=0, y \neq 0$, et le troisième avee $q(y) \neq 0$. Cela nous donne:

$$
\alpha(V \boxplus H)=p \cdot \alpha(V)+\left(p^{n}+1\right)(p-1)
$$

ou encore:

$$
\alpha(V \boxplus I I)-p^{n \cdot 1}+1=p \cdot\left(\alpha(V)-p^{n \cdots 1}+1\right)
$$

Donc, si $V$ est du type $(A)$ et premier quelconque, c'est-à-dire si $V=m \cdot H$, on obticnt par récurrence:

$$
\begin{aligned}
\alpha(V)-p^{2 m \cdot 1}+1 & =p \cdot\left(\alpha((m-2) \cdot H)-p^{2 m-3}+1\right) \\
& =p^{2} \cdot\left(\alpha((m-1) \cdot H)-p^{2 m-5}+1\right)=\cdots \\
& =p^{2 n} \cdot(\underbrace{\alpha(0 \cdot H)}_{=0}-p^{-1}+1) .
\end{aligned}
$$

Ce qui nous donne:

$$
\alpha(V)=p^{2 m-1}-1-p^{m \cdot 1}+p^{m}=\left(p^{m}-1\right)\left(p^{m-1}+1\right) .
$$


Chapitre 3 : Le groupe orthogonal sur les corps $\mathbb{F}_{p}$.

Maintenant, si $V=(m-1) \cdot H \boxplus J, J$ défini comme au lemme 3.5, on a :

$$
\left.\alpha(V)-p^{2 m-1}+1=p \cdot \alpha((m-1) \cdot H \boxplus J)-p^{2 n-3}+1\right)=\cdots=p^{m-1} \cdot(\underbrace{\alpha(J)}_{=0}-p+1)=p^{m-1}-p^{m}
$$

donc:

$$
\alpha(V)=p^{2 m}{ }^{2}-1+p^{m \cdot 1}-p^{r n}=\left(p^{m}+1\right)\left(p^{m-1}-1\right) .
$$

Finalement, si $V$ est de ype (C), de rang $2 m+1$ et $p$ premier impair, on trouve:

$$
\alpha(V)-p^{2 m}+1=p \cdot\left(\alpha((m-1) \cdot H \boxplus I)-p^{2 m-2}+1\right)=\cdots=p^{m} \cdot(\underbrace{\alpha(I)}_{=0}-p^{0}+1)=0
$$

d'où :

$$
\alpha(V)=p^{2 m}-1
$$

\section{Théorème 3.8}

Soient $(V, q)$ un espace quadratique non dégénéré sur ${ }_{p}$ de dimension $n=2 m$ ou $2 m+1$ selon la parité de la dimension de $V$, et $O_{q}^{n}(V)$ le groupe orthogonal associé. On a :

$\left|O_{q}^{n}(V)\right|= \begin{cases}2 \cdot p^{\frac{n(n-1)}{2}}\left(1-p^{m}\right) \prod_{0<2 i<n}\left(1-p^{2 i}\right) & \text { si } V \text { est du type }(A) \text { de rang } 2 m \text { et } p \text { premier quelconque } \\ 2 \cdot p^{\frac{n(n-1)}{2}}\left(1+p^{m}\right) \prod_{0<2 i<n}\left(1-p^{2 i}\right) & \text { si } V \text { est du type (B) de rang } 2 m \text { et } p \text { premier impair } \\ 2 \cdot p^{\frac{n(n-1)}{2}} \prod_{0<2 i<n}\left(1-p^{2 i}\right) & \text { si } V \text { est du type (C) de rang } 2 m+1 \text { et } p \text { premier impair. }\end{cases}$

\section{Dérnonstration :}

On cherche à nouveau une relation entre $\left|O_{q}(V \pm H)\right|$ et $\left|O_{q}(V)\right|$. Pour se fixer les idées, on pose $H=$ $\mathbb{E}_{p} \cdot h_{1}+\mathbb{H}_{p} \cdot h_{2}$, où $q\left(h_{1}\right)=q\left(h_{2}\right)=0$ et $\beta_{q}\left(h_{1}, h_{2}\right)=1$. Soit aussi

$$
\begin{gathered}
\varphi: O_{q}(V \boxplus H) \longrightarrow\{x \in V \backslash\{0\} \mid q(x)=0\} \\
u \longmapsto u\left(h_{1}\right) .
\end{gathered}
$$

$\varphi$ est surjective en vertu du théorimo de Witt. $u, u^{\prime}$ ont même image si et seulement si $u^{-1} u^{\prime} \in\{u \in$ $\left.O_{q}(V \boxplus H) \mid u\left(h_{1}\right)=h_{1}\right\}$. Donc, les classes à gauche de ce groupe sont en bijection avec l'ensemble des vecteurs non muls de longueur mulle. Airisi, on a :

$$
\left|O_{q}(V \boxplus H)\right|=\alpha(V \boxplus H) \cdot\left|\left\{u \in O_{q}(V \boxplus H) \mid u\left(h_{1}\right)=h_{1}\right\}\right| .
$$

Nous allons maintenant nous donner uno nouvelle surjection :

$$
\begin{aligned}
y:\left\{u \in O_{q}(V \boxplus H) \mid u\left(h_{1}\right)=h_{1}\right\} & \longrightarrow \mathscr{F} \\
u & \longrightarrow u\left(h_{2}\right),
\end{aligned}
$$

où F est l'ensemble des $h_{2}^{\prime} \in V \boxplus / /$ lek que $q\left(h_{2}^{\prime}\right)=0$ et $\beta_{q}\left(h_{1}, h_{2}^{\prime}\right)=1$. Comme avant, $u$ et $u^{\prime}$ ont mòme image si et seulement si $u^{\prime} u^{\prime} \in\left\{u \in O_{q}(V \boxplus H) \mid u\left(h_{1}\right)=h_{1}, u\left(h_{2}\right)=h_{\iota_{2}}\right\}$; mais cet ensemble est en bijection ave: $O_{1 j}(V)$, sacharut que le theoreme de Wit. est valable dans notre cas.

Intéressons-nous maintenant au cardinal de

A priori, un élément $h_{2}^{\prime}$ de $\mathscr{G}$ sécrit $v+a h_{1}+b h_{2}, v \in V$ et $a, b \in{ }_{p}$. Or $1=\beta_{q}\left(h_{1}, h_{2}^{\prime}\right)=b$. On a aussi que $q\left(h_{2}^{\prime}\right)=q(v)+a b=q(v)+a$. 
Pour chaque $v$ choisi, il n'y a donc qu'un a possible et un seul. Puisque le nombre de $v$ est $p^{n}$, on obtient alors la formule :

$$
|O(V \boxplus H)|=\alpha(V \boxplus H) \cdot p^{n} \cdot|O(V)| .
$$

Nous pouvons donc examincr chaque cas:

type (A) : vu le lemme 3.7 , on a:

$$
\begin{aligned}
\left|O_{q}(m \cdot H)\right|= & \left(p^{m}-1\right)\left(p^{m-1}+1\right) p^{2 m-2}|O((m-1) \cdot H)| \\
= & p^{(2 m-1) \cdot(2 m-2)}\left(\left(1-p^{\cdot m}\right)\left(1+p^{-(m-1)}\right)\left|O_{q}((m-1) \cdot H)\right|\right. \\
= & p^{(2 m-1) \cdot(2 m-2) \cdot(2 m \cdot 3):(2 m \cdot 1)}\left(1-p^{-m}\right)\left(1+p^{-(m-1)}\right)\left(1-p^{(m-1)}\right)\left(1+p^{-(m-2)}\right) . \\
& \left.\quad \cdot(1-p)^{(m-2)}\right)\left|O_{q}((m-2) \cdot H)\right| \\
& \quad \vdots \\
= & p^{(2 m-1)+(2 m-2) \cdots \cdots+1}\left(1-p^{-m n}\right) \prod_{0<2 i<n}\left(1-p^{-2 i}\right) \cdot 2 \\
= & 2 \cdot p^{\frac{n(n-1)}{2}(1-p \cdot m)} \prod_{0<2 i<n}\left(1-p^{-2 i}\right) .
\end{aligned}
$$

type (B) : Si $V=(m-1) \cdot H \boxplus J$, on obtient :

$$
\begin{aligned}
\left|O_{q}(V)\right|= & \left(p^{m}+1\right)\left(p^{m-1}-1\right) p^{2 m-2}\left|O_{q}((m-2) \cdot H \boxplus J)\right| \\
& \vdots \\
= & p^{(2 m-1) \cdots(2 m-2) \cdots 2^{2}}\left(1+p^{-m}\right)\left(1-p^{-(m-1)}\right) \cdots\left(1+p^{-2}\right)\left(1-p^{-1}\right)|O(J)| .
\end{aligned}
$$

Le problème maintenant est de calculer $|O(J)|$.

Rappelons que $J$ est un espace de dimension 2 de base $\left(e_{1}, e_{2}\right)$ tel que $\beta_{q}\left(e_{1}, e_{2}\right)=0, q\left(e_{1}\right)=1$ et $q\left(e_{2}\right)=a$ avec $a \notin w_{p}^{2}$.

Commençons par calculer le nombre de vecteurs de longueur 1 , c'est-à-dire le nombre de solutions de l'équation $x^{2}+a y^{2}=1$. On peut wir le membre de gauche comme la norme de $x+y \sqrt{-a}$ dans $\mathbb{E} p(\sqrt{-a})$ qui a $p^{2}$ éléments. Donc, les vecturs de norme 1 peuvent cure vus comme le noyal de l'application $N: w_{p}(\sqrt{-a})^{*} \rightarrow$, délinie par $N(x+y \sqrt{-a})=x^{2}+a y^{2}$. Le cardinal de ce noyau est évidemment $\frac{p^{2}-1}{p-1}=p+1$. De plus, l'application

$$
\begin{aligned}
\rho: O(J) & \longrightarrow \operatorname{ker}(N) \\
u & \longmapsto u\left(e_{1}\right)
\end{aligned}
$$

est surjective et $\rho(u)=\rho\left(u^{\prime}\right)$ si et seulement si $u^{-1} u^{\prime}\left(e_{1}\right)=e_{1}$. On remarque que compter les $u$ dans $O(J)$ tels que $u\left(c_{1}\right)=c_{1}$ reviont à compter le nombre de couples $(x, y)$ de longueur a et orthogonaux à $e_{1}$. Il faut donc que $x=0$ el par suite $a y^{2}=a$ donc $y= \pm 1$. Cela nous fait alors deux possibilités pour $y$ (on a supposé $p \neq 2$ ). Il suit :

$$
|O(J)|=2(p+1)
$$

Tenant compte de la formule (II), on obtient:

$$
|O(V)|=2 \cdot p^{\left.\frac{n(n \cdot n}{2} \cdot 1\right)}\left(1+p^{-m}\right) \prod_{0<22<n}\left(1-p^{-22}\right) .
$$

Remarque: Si $p=2$ et si f J est lepace muni de la forme $x^{2}+x y+y^{2}$, on voit aisément que $|O(J)|=3$, et on obticendrait donc la meme formuke, mais avec un lactear 2 cn moins. 
Type (C) : Si $V=m \cdot H \boxplus\langle a\rangle$, puisclue $p$ est impair, on a que $\mid O(<a\rangle) \mid=2$. Ce qui nous donne :

$$
\begin{aligned}
|O(E)|= & p^{2 m \cdot 1}\left(p^{2 m}-1\right)|O((m-1) \cdot H \boxplus<a>)| \\
= & p^{2 m \cdot(2 m}{ }^{1)}\left(1-p^{2 m}\right) \mid O((m-1) \cdot H \boxplus<a>\mid \\
& \vdots \\
= & 2 \cdot p^{\frac{n(n-1)}{2}} \prod_{0<2 i<n}\left(1-p^{-2 i}\right) .
\end{aligned}
$$

\section{Corollaire 3.9}

Si $V$ est de dimension $n=2 m$ et si $p$ est impair, alors :

$$
\left|O_{q}^{n}(V)\right|=2 \cdot p^{\frac{n(n-1)}{2}}\left(1-\left(\frac{d}{p}\right) p^{-m}\right) \prod_{0<2 i<n}\left(1-p^{-22}\right) .
$$

Où $d=(-1)^{\frac{n(n-1)}{2}} \operatorname{det}(V)$ est le discriminant de $V$, et $(\underset{p}{p})$ est le symbole de Legendre.

\section{Démonstration :}

On voit que le type $(A)$ correspond à $(-1)^{m} \operatorname{det}(V) \in *_{p}^{*}$

et que le type(B) correspond à $(-1)^{m} \operatorname{det}(V) \notin \notin_{p}^{2}$. De plus $\frac{n(n-1)}{2}=m(n-1) \equiv \operatorname{mom} \bmod 2$. 


\section{CHAPITRE 4 \\ Le groupe orthogonal modulo 8.}

Le but de ce chapitre est de calculer le cardinal du groupe orthogonal pour la forme $\beta=x_{1} y_{1}+\cdots+x_{n} y_{n}$, définie sur un $\mathbb{Z} / 8 \mathbb{Z}$-module libre de rang $n$.

\section{A. Groupes orthogonaux quadratiques et bilinéaires.}

\section{Définition 4.1}

Soit $M$ un $\mathbb{Z} / 8 \approx$-module libre de rang $n$ muni d'une forme quadratique $q$. Le groupe orthogonal quadratique modulo 8 est le groupe $O_{q}^{n}(\mathbb{Z} / 8 \mathbb{Z})$ formé des isomorphismes $u: M \rightarrow M$ tels que $q(u(x))=q(x)$ pour tout $x \in M$.

\section{Définition 4.2}

Soit $M$ un $\mathbb{Z} / 8 \leftleftarrows-$-module libre de rang $n$ muni d'une forme bilinéaire $\beta$. Le groupe orthogonal bilinéaire modulo 8 est le groupe $O_{\beta}^{n}(-/ 8)$ formé des isomorphismes $u: M \rightarrow M$ tels que $\beta(u(x), u(y))=\beta(x, y)$ pour tout $x, y \in M$.

\section{Proposition 4.3}

Si $\beta(x, y)=x_{1} y_{1}+\cdots+x_{n} y_{n}$ et $q(x)=x_{1}^{2}+\cdots+x_{n}^{2} \quad x, y \in M$, on a $\left|O_{q}^{n}(\mathbb{Z} / 8 \mathbb{Z})\right|=2^{n(n \cdot 1) / 2} \cdot\left|O_{\beta}^{n}(\cdots / 8)\right|$

\section{Démonstration :}

Notons $O_{q}^{n}$ pour $O_{q}^{n}(\mathbb{E} / 8 \ldots)$ et $O_{\beta}^{n}$ pour $O_{\beta}^{n}(m / 8 \mathbb{Z})$.

Il est clair que $O_{\beta}^{n}=\left\{O \in \mathrm{M}_{n}\left(\% / 8^{\infty}\right) \mid O O^{t}=I_{n}\right\}$ et que $O_{q}^{n}=\left\{O \in \mathrm{M}_{n}(\mathbb{Z} / 8 \mathbb{Z}) \mid O O^{t}=I_{n}+4 S, S \in \mathscr{S}\right\}$ où $\mathscr{S}$ est l'ensemble des matrices $\left(S_{i j}\right)_{i, j \in \mathbb{N}_{n}}$ telles que $S_{i j}=S_{j i} \in \mathbb{Z} / 2 \mathbb{Z}$ et $S_{i i}=0$ pour tout $i, j \in \mathbb{I}_{n}$. On a donc que $O_{\beta}^{n}$ est un sous-groupe de $O_{q}^{n}$. Soit $\phi: O_{q}^{n} \rightarrow \mathscr{S}$, définie par $\phi(O)=S$ où $4 S=O O^{t}-I_{n}$. Nous allons voir que $\phi$ est une application surjective et qu'elle induit une bijection entre $O_{q}^{n}$ modulo $O_{\beta}^{n}$ et $\mathscr{S}$.

$\phi$ est surjective, car si $S \in \mathscr{S}$, on a $I_{n}+4 S=\left(I_{n}+4 S^{+}\right)\left(I_{n}+4 S^{+}\right)^{t}$ où $S^{+}$est la matrice triangulaire supérieure formée des coefficients au-dessus de la diagonale de $S$.

Si $O_{1}, O_{2} \in O_{q}^{n}$ sont tels que $O_{2} O_{2}^{t}=O_{1} O_{1}^{t}=I_{n}+4 S$, alors $O_{2} O_{2}^{t} O_{1}^{t^{-1}}=O_{1}$. Donc $O_{1}^{-1} O_{2} O_{2}^{t} O_{1}^{t^{-1}}=$ $\left(O_{1}^{-1} O_{2}\right)\left(O_{1}^{-1} O_{2}\right)^{t}=I_{n}$ ce qui nous donne que $O_{1}^{-1} O_{2} \in O_{\beta}^{n}$. Réciproquement, si $O_{1}^{-1} O_{2} \in O_{\beta}^{n}$, alors $O_{1} O_{1}^{t}=O_{2} O_{2}^{t}$ et donc $\phi\left(O_{1}\right)=\phi\left(O_{2}\right)$

$O_{\beta}^{n}$ induit une relation d'équivalence: $O_{1} \sim O_{2}$ si $O_{1}^{-1} O_{2} \in O_{\beta}^{n}$.

On a finalement une bijection : $O_{q}^{n} / \sim \rightarrow \mathscr{F}, O \mapsto \phi(O), O \in \bar{O}$.

Puisque $|\mathscr{P}|=2^{n(n-1) / 2}$, on conclut.

\section{Remarque :}

Nous n'utiliserons ce résultat que dans une version atténuée lors de la proposition 5.4 , à savoir que $O_{\beta}^{n}$ est contenu strictement dans $O_{q}^{n}$. 


\section{B. Les vecteurs de norme $i$.}

Dans ce pragraphe, nous allons calculer le cardinal des vecteurs de norme $i$ pour $i$ fixé dans $\mathbb{Z} / 8 \mathbb{Z}$.

Pour des raisons techniques, il est bon de se fixer quelques notations:

$t_{i}(n):=\left|\left\{x \in(\mathbb{Z} / 8 \mathbb{Z})^{n} \mid x_{1}^{2}+\cdots+x_{n}^{2}=i\right\}\right| \quad n \in \mathbb{I}$ if et $i \in \mathbb{Z} / 8 \mathbb{Z}$

$u_{1}(n):=t_{1}(n)-t_{3}(n)+t_{5}(n)-t_{7}(n)$

$u_{0}(n):=t_{0}(n)-t_{2}(n)+t_{4}(n)-t_{6}(n)$

$\epsilon_{i}(n):=t_{i}(n)-t_{i+4}(n) \quad i=0,1,2,3$

$s_{i}(n):=t_{i}(n)+t_{i+4}(n) \quad i=0,1,2,3$

Si on fixe une variable, on obtient aisément la relation :

$$
\text { (*) } \quad t_{i}(n+1)=2 t_{i}(n)+4 t_{i-1}(n)+2 t_{i-4}(n) \quad \forall i \in \mathbb{Z} / 8 \mathbb{Z}
$$

car $1,3,5$ et 7 sont de carré $1 \bmod 8,0$ et 4 sont de carré $0 \bmod 8$; enfin, 2 et 6 sont de carré $4 \bmod 8$. Ceci nous donne l'équation matricielle:

$$
\begin{gathered}
T(n+1)=A \cdot T(n) \\
\text { où } T(n)=\left(\begin{array}{c}
t_{0}(n) \\
t_{1}(n) \\
\vdots \\
t_{7}(n)
\end{array}\right) \text { et } A=\left(\begin{array}{cccccccc}
2 & 0 & 0 & 0 & 2 & 0 & 0 & 4 \\
4 & 2 & 0 & 0 & 0 & 2 & 0 & 0 \\
0 & 4 & 2 & 0 & 0 & 0 & 2 & 0 \\
0 & 0 & 4 & 2 & 0 & 0 & 0 & 2 \\
2 & 0 & 0 & 4 & 2 & 0 & 0 & 0 \\
0 & 2 & 0 & 0 & 4 & 2 & 0 & 0 \\
0 & 0 & 2 & 0 & 0 & 4 & 2 & 0 \\
0 & 0 & 0 & 2 & 0 & 0 & 4 & 2
\end{array}\right) \\
\text { Donc } \left.T(n+1)=A^{n} \cdot T(1) \text { et } T(1)=\left(\begin{array}{l}
2 \\
4 \\
0 \\
0 \\
2 \\
0 \\
0 \\
0
\end{array}\right) \text { } \forall n \in \Pi\right]
\end{gathered}
$$

Les équations précédentes donnent alors, grâce à un bref raisonnement par récurence, que la première colonne de $A^{n}$ n'est autre que $T(n) \mathrm{cl}$, comme $A$ est une matrice "circulante", $A^{n}$ aussi. Donc, la première ligne de $A^{n}$ est $\left(t_{0}(n), t_{7}(n), \ldots, t_{1}(n)\right)$.

Le polynôme caractéristique de $A$ (qui est aussi le polynóme minimal) est : $X\left(X^{4}+256\right)\left(X^{2}-8 X+\right.$ $32)(X-8)$.

Calculons le noyau de $A^{2}-8 A+32 l_{8}$ qui sera bien sûr contenu dans celui de $A^{n+1}-8 A^{n}+32 A^{n-1}$ pour tout $n \in \mathrm{I}$. Il est engendré par les deux vecteurs suivants:

$$
(0,-1,0,1,0,-1,0,1) \text { et }(1,0,-1,0,1,0,-1,0) \text {. }
$$

Ce qui nous donne les deux équations suivantes:

$$
u_{i}(n+1)=8 u_{i}(n)-32 u_{i}(n-1) \quad i=0,1
$$

En itérant 2 fois cette formule, on obtient : $u_{i}(n+4)=-2{ }^{10} u_{i}(n)$. Donc:

$$
u_{i}(j+4 k)=(-1)^{k_{2}} 2^{10 k} u_{i}(j) \text { pour } 1 \leq j \leq 4, i=0,1 \text {, et } k \in[\mathrm{J} .
$$


Regardons le noyau de $A^{4}+256 I_{8}$, donc une partie de celui de $A^{n+3}+256 A^{n-1}$ pour tout $n \in \mathrm{I}$. On trouve l'espace :

$$
<(0,0,0,1,0,0,-1),(0,0,1,0,0,0,-1,0),(0,1,0,0,0,-1,0,0),(1,0,0,0,-1,0,0,0)>\text {. }
$$

Cela nous donne les équations suivantes : $\epsilon_{i}(n+4)=-256 \epsilon_{i}(n)$ pour $i=0,1,2,3$ d'où :

$$
\epsilon_{i}(j+4 k)=(-1)^{k} 2^{8 k} \epsilon_{i}(j) \text { pour } 0 \leq i \leq 3, k \in \text { IJ et } j \in \Pi_{4} .
$$

Le noyau de $A$ lui-même est l'espace engendré par $(-1,1,-1,1,-1,1,-1,1)$. On trouve alors : $s_{0}(n)+$ $s_{2}(n)=s_{1}(n)+s_{3}(n)$. Il est clair que $\sum_{i=0}^{4} s_{i}(n)=8^{n}$, donc :

$$
s_{i}(n)+s_{i \cdot 2}(n)=\frac{8^{n}}{2} \quad \text { pour } i=1,2 .
$$

Il est évident que $u_{i}(n)=s_{i}(n)-s_{i * 2}(n)$ pour $i=0,1$. Sachant cela et vu les équations $\left(I^{\prime}\right)$ et $(I I I)$, on obtient le système :

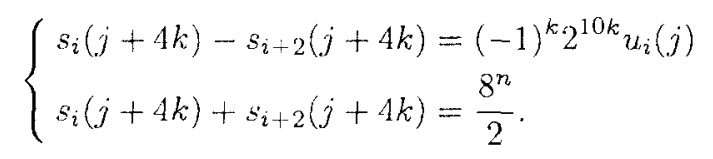

Ce qui fait :

$$
s_{i}(j+4 k)=t_{i}(j+4 k)+\iota_{i+4}(j+4 k)=(-1)^{k_{2}} 2^{10 k-1} u_{i}(j)+\frac{8^{j+4 k}}{2^{2}} .
$$

En se souvenant de la définition de $\epsilon_{i}(j+4 k)$ et gràce aux équations $(I I)$ et $(I V)$, on a:

$$
t_{i}(j+4 k)=(-1)^{k_{2}} 2^{8 k \cdot 1}\left(2^{2 k \cdots 1} u_{i}(j)+\epsilon_{i}(j)\right)+8^{j+4 k-1} \quad i=0,1 \text { et } j \in \mathrm{H}_{4}
$$

Nous sommes donc en mesure de démontrer la

\section{Proposition 4.4}

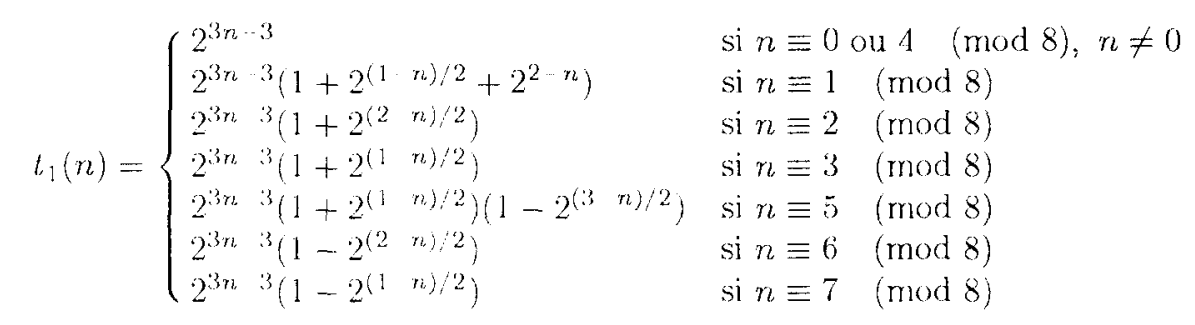

\section{Dérnonstration :}

Si $\mathbf{n}=1+4 \mathrm{k}$, on a :

$u_{1}(1)=t_{1}(1)-t_{3}(1)+t_{5}(1)-\iota_{7}(1)=4+0+0+0=4$ et $\epsilon_{1}(1)=t_{1}(n)-t_{5}(1)=4$. Donc, l'équation $(A)$ devient :

$$
\begin{aligned}
t_{1}(1+4 k) & =(-1)^{k} 2^{8 k-1}\left(2^{2}\left(2^{2 k-1}+1\right)\right)+8^{j+1 k \cdots 1}=2^{8 k-1}\left((-1)^{k}\left(2^{2 k \cdots 1}+1\right)+2^{4 k-1}\right) \\
& =2^{2 n-1}\left((-1)^{(n-1) / 4}\left(2^{(n-3) / 2}+1\right)+2^{n-2}\right) .
\end{aligned}
$$

On en déduit que si $n \equiv 1$ (mod 8 ), alors:

$$
t_{1}(n)=2^{2 n-1}\left(2^{n-2}+2^{(n-3) / 2}+1\right)=2^{3 n-3}\left(1+2^{(1-n) / 2}+2^{2--n}\right),
$$

et que si $n \equiv 5 \quad(\bmod 8)$, on obtisnt

$$
\iota_{1}(n)=2^{3 n}{ }^{3}\left(1-2^{(1 \quad n) / 2}-2^{2} n\right)=2^{3 n \cdots 3}\left(1+2^{(1-n) / 2}\right)\left(1-2^{(3-n) / 2}\right) .
$$


Si $\mathbf{n}=\mathbf{2}+4 \mathrm{k}$, on $\mathrm{a}$ :

$u_{1}(2)=2^{4}+2^{4}=2^{5}$ et $\epsilon_{1}(2)=2^{4}-2^{4}=0$; donc

$$
\begin{aligned}
& t_{1}(n)=(-1)^{k} 2^{8 k-1}\left(2^{2 k-1} \cdot 2^{5}\right)+8^{4 k+1}=2^{10 k+3}\left((-1)^{k}+2^{2 k}\right) \\
& =2^{(5 n-1) / 2}\left(2^{(n-2) / 2}+(-1)^{(n-2) / 4}\right) .
\end{aligned}
$$

Si $n \equiv 2(\bmod 8)$, on a :

$$
t_{1}(n)=2^{(5 n-4) / 2}\left(2^{(n-2) / 2}+1\right)=2^{3 n-3}\left(1+2^{(2-n) / 2}\right)
$$

et si $n \equiv 6 \quad(\bmod 8)$, on a

$$
t_{1}(n)=2^{3 n-3}\left(1-2^{(2-n) / 2}\right)
$$

Pour le cas où $\mathbf{n}=3+4 \mathrm{k}$, on a : $u_{1}(3)=3 \cdot 2^{5}-2^{6}+3 \cdot 2^{5}-0=2^{7}$ et $\epsilon_{1}(2)=3 \cdot 2^{5}-3 \cdot 2^{5}=0$, donc

$$
\begin{aligned}
t_{1}(n) & =(-1)^{k} 2^{8 k-1}\left(2^{2 k-1} \cdot 2^{7}\right)+8^{4 k+2}=2^{10 k+5}\left((-1)^{k}+2^{2 k+1}\right) \\
& =2^{(5 n \cdots 5) / 2}\left(2^{(n+1) / 2}+(-1)^{(n-3) / 4}\right) .
\end{aligned}
$$

Done, si $n \equiv 3 \quad(\bmod s)$, on a :

$$
t_{1}(n)=2^{(5 n \cdot 5) / 2}\left(2^{(n+1) / 2}+1\right)=2^{3 n-3}\left(1+2^{(1-n) / 2}\right)
$$

et si $n \equiv 7 \quad(\operatorname{mrod} 8)$, on trouve :

$$
t_{1}(n)=2^{3 n-3}\left(1-2^{(1-n) / 2}\right)
$$

Finalement, si $\mathrm{n}=4 \mathrm{k}+4$, on a :

$u_{1}(4)=2^{9}-2^{9}+2^{9}-2^{9}=0$ et $c_{1}(4)=2^{9}-2^{9}=0$, d'où :

$$
t_{1}(n)=8^{n-1}=2^{3 n-3}
$$

\section{Rernarques :}

a) Les petites valeurs de $u_{1}(n)$ ct de $\epsilon_{1}(n)$ ont bien sûr été calculées grâce à l'équation $T(n+1)=A^{n} T(1)$.

b) Par la suite, nous aurons essentiellement besoin des $t_{1}(n)$ sous la forme donnée à la proposition précédente, mais les équations $(I)-(I V)$ et $(A)$ nous permettant de calculer les autres $t_{i}(n)$, nous obtiendrons une formule plus compacte que la précédente, à savoir :

Proposition 4.5

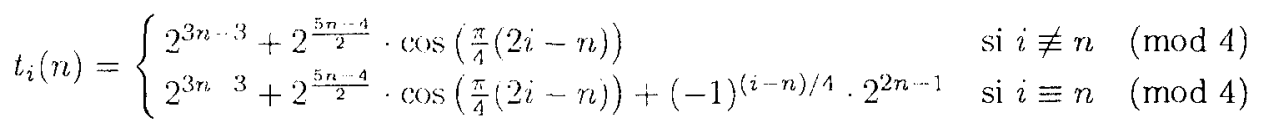

\section{Démonstration :}

Une rapide vérification nous permet de voir que la formule marche pour $t_{1}(n)$.

Commençons tout d'abord par calculer $t_{0}(n)$ :

Si $\mathrm{n}=1+4 \mathrm{k}: u_{0}(1)=t_{0}(1)-\iota_{2}(1)+t_{4}(1)-t_{6}(1)=2+2=4$ et $\epsilon_{0}(1)=t_{0}(1)-t_{4}(1)=0$.

Donc vu $(A)$, on a :

$$
\begin{aligned}
\iota_{0}(n) & =\left((-1)^{(n \cdot 1) / 4} \cdot 2^{(5 n-5) / 2}+2^{3 n-3}\right) \\
& =\left(2^{3 n-3}+2^{(5 n-5) / 2}\right)
\end{aligned}
$$

si $n \equiv 1 \quad(\bmod 8)$. Et ,

$$
t_{0}(n)=\left(2^{3 n-3}-2^{(5 n-5) / 2}\right)
$$


si $n \equiv 5 \quad(\bmod 8))$.

Si $\mathbf{n}=2+4 \mathrm{k}$ :

$u_{0}(2)=2^{3}-2^{4}+2^{3}=0$ et $c_{0}(2)=2^{3}-2^{3}=0$ donc $t_{0}(n)=2^{3 n-3}$.

Si $\mathbf{n}=3+4 k$ :

$u_{0}(3)=2^{5}-3 \cdot 2^{5}+2^{5}-3 \cdot 2^{5}=-2^{7}$ el $\epsilon_{0}(3)=0$.

Donc, on a $\left.t_{0}(n)=\left((-1)^{(n \cdot 1) / 4} \cdot 2^{(5 n \cdot 5) / 2}+2^{3 n \cdot 3}\right)=\left(2^{3 n-3}-2^{(5 n} 5\right) / 2\right)$ si $n \equiv 3 \quad(\bmod 8)$ (respectivement $\left(2^{3 n-3}+2^{(5 n \cdot 5) / 2}\right)$ si $\left.n \equiv 7 \quad(\bmod 8)\right)$.

Si $\mathrm{n}=4+4 \mathrm{k}$ :

$u_{0}(4)=2^{7}-3 \cdot 2^{8}+3 \cdot 2^{7}-3 \cdot 2^{8}=-2^{10}$ et $\epsilon_{0}(3)=2^{7}-3 \cdot 2^{7}=-2^{8}$.

Donc, on a $t_{0}(n)=\left((-1)^{n / 4}\left(2^{(5 n-4) / 2}+2^{2 n-1}\right)+2^{3 n-3}\right)=\left(2^{3 n-3}+2^{(5 n-4) / 2}+2^{2 n-1}\right)$ si $n \equiv 0 \quad(\bmod 8)$

(respectivement $\left(2^{3 n-3}-2^{(5 n-4) / 2}+2^{2 n-1}\right)$ si $\left.n \equiv 4 \quad(\bmod 8)\right)$.

La relation $(*)$ nous donne $: t_{1}(n+1)=2 t_{1}(n)+4 t_{0}(n)+2 t_{5}(n)$. On trouve alors facilement $t_{5}(n)$. Puis, en faisant varier judicieusement $i$ dans $(*)$, on trouve les autres $t_{i}(n)$.

E. Preissmann et H. Joris mont aimablement donné d'autres démonstrations plus analytiques de ce résultat qui par ailleurs est cité dans $[8, \mathrm{ch} . / /, 89]$. Le lecteur trouvera ces démonstrations dans l'appendice de ce travail.

\section{Le cardinal du groupe $O_{\beta}^{n}$.}

Dans ce paragraphe, nous allons donner une formule calculatoire pour $\left|O_{\beta}^{n}\right|$; mais pour $y$ arriver, il faudra établir une forme "canonique" à toute forme bilinéaire non dégénérée sur *.../8 $/ 8$, puis chercher les vecteurs de norme 1 qui ont des supplémontaires orthogonaux pairs. Ces vecteurs seront appelés "mauvais vecteurs", car le theoròne de: Wiu ne sapplique pas pour eux.

\section{Lemme 4.6}

Soit $(V, \beta)$ une forme bilinéaire de dimension $n$ sur $-/ 8$. Si $x \in V$ est tel que $\beta(x, x)=a$ impair (donc inversible), alors $\beta \simeq\langle a>\boxplus U$. D'autre part, si $\beta(x, x)$ et $\beta(y, y)$ sont pairs mais que $\beta(x, y)$ est impair, alors $V \simeq\langle x, y\rangle \boxplus\langle x, y\rangle^{-}$.

\section{Démonstration :}

a) Si $f$ est une application linéaire do $\langle x\rangle$ dans $-2 / 8 z$, il est clair que $\beta\left(\frac{x \cdot f(x)}{\beta(x, x)}, \cdot\right)=f$, donc $\left.\beta\right|_{<x>}$ est non dégénérée. La proposition 1.5 nous donne: $V \simeq\langle x\rangle \boxplus\langle x\rangle^{-1}$.

b) Soit $f:\langle x, y\rangle \rightarrow-/ 8$ linéaire. Pour prouver que $\left.\beta\right|_{\langle x, y\rangle}$ est non dégénérée, il suffit de trouver un $z=\lambda x+\mu y$ tel que $\beta(z, x)=f(x)$ el $\beta(z, y)=f(y)$, ce qui nous donne le système suivant à résoudre :

$$
\left\{\begin{array}{l}
\lambda \beta(x, x)+\mu \beta(y, x)=f(x) \\
\lambda \beta(x, y)+\mu \beta(y, y)=f(y)
\end{array}\right.
$$

En posant $\mu=f(x) \beta(x, y)(1-\beta(x, x) \beta(y, y))-f(y) \beta(x, x)$ et $\lambda=\beta(x, y)(f(y)-\mu \beta(x, y))$, on conclut grâce à la proposition 1.5

\section{Corollaire 4.7}

Toute forme bilinéaire symétrique non dégénéré sur $\frac{1}{2} / 8$ est isomorphe à une forme du type :

$$
<a_{1}>\boxplus \cdots \boxplus<a_{j}>\boxplus\left\langle\left(\begin{array}{ll}
b_{1} & d_{1} \\
d_{1} & c_{1}
\end{array}\right)>\boxplus \cdots \boxplus<\left(\begin{array}{cc}
b_{i} & d_{i} \\
d_{i} & c_{i}
\end{array}\right)>,\right.
$$

ò̀ $a_{k}$ impair $\forall k$ et det $\left(\begin{array}{ll}b_{l} & d_{l} \\ d_{l} & c_{l}\end{array}\right)$ impair $\forall l$. 


\section{Démonstration :}

S'il existe $x_{1}$ tel que $\beta\left(x_{1}, x_{1}\right)=a_{1}$ impair, alors par le lemme précédent : $\beta \simeq<a_{1}>\boxplus V$. Et on refait le même raisonnement jusqu'à ce que $\beta \simeq\left\langle a_{1}>\boxplus \cdots \boxplus\left\langle a_{j}\right\rangle \boxplus V^{\prime}\right.$ ò̀ $\beta(x, x)$ pair $\forall x \in V^{\prime}$. Comme $\beta$ est non dégénérée, $\exists x_{1}, y_{1}$ tels que $\beta\left(x_{1}, y_{1}\right)$ impair, donc par le lemme précédent :

$$
\beta \simeq<a_{1}>\boxplus \cdots \boxplus<a_{j}>\boxplus<\left(\begin{array}{ll}
\beta\left(x_{1}, x_{1}\right) & \beta\left(x_{1}, y_{1}\right) \\
\beta\left(x_{1}, y_{1}\right) & \beta\left(y_{1}, y_{2}\right)
\end{array}\right)>\boxplus V^{\prime \prime}
$$

et on termine par récurrence.

\section{Corollaire 4.8}

Si $(V, \beta)$ est non dégénérée et de dimension impaire, alors il existe $x \in V$ tel que $\beta(x, x)$ impair. On dit alors par convention que $V$ est impatr, sinon, $V$ est dit pair. •

\section{Lemme 4.9}

Supposons que $E \boxplus V \simeq E \boxplus V^{\prime}$ et que $E, V$ et $V^{\prime}$ sont impairs, alors $V \simeq V^{\prime}$.

\section{Démonstration :}

Cette version du théorème de Witt ost démontréc dans $[2$, thm 4.5, pp. 82-83]

\section{Lemme 4.10}

Soit $(V, \beta)$ tel que $M_{\beta}=I_{n}$ ot $n \neq 1$ (mod 8$)$. Si $x \in V$ est tel que $\beta(x ; x)=1$, alors il existe $U$ et $y \in U$ tels que $V \simeq\langle x\rangle \boxminus U$ et,$\beta(y, y)$ impair

\section{Démonstration :}

Par le lemme 4.6, on sait qu'il existe $U$ avec $V \simeq\langle x\rangle \boxplus U$. Jar le corollaire 4.8, si $n$ est pair, donc si $\operatorname{dim}(U)$ est impair, le lemmo est prouvé. Si $n$ est impair et $n \neq 1$ (mod 8), on a que $\operatorname{dim}(U)$ est paire et $\operatorname{det}\left(U,\left.\beta\right|_{\ell}\right)=1$. Supposons que $U$ ne représente que des éléments pairs, alors :

$$
U \simeq<\left(\begin{array}{cc}
a_{1} & c_{1} \\
c_{1} & b_{1}
\end{array}\right)>\boxplus \cdots \boxplus<\left(\begin{array}{cc}
a_{(n-1) / 2} & c_{(n-1) / 2} \\
c_{(n-1) / 2} & b_{(n-1) / 2}
\end{array}\right)>
$$

avec det $\left(\begin{array}{cc}a_{i} & c_{i} \\ c_{i} & b_{i}\end{array}\right)$ impair. Mais puisque $a_{i}, b_{i}$ sont pairs $\forall i$, on a que $\operatorname{det}\left(\begin{array}{ll}a_{i} & c_{i} \\ c_{i} & b_{i}\end{array}\right)=-1$ ou 3 , et $c_{i}$ est impair $\forall i$.

Soient $\lambda$ et $\alpha$ impairs, grâce aux matrices de changoment de base $\left(\begin{array}{cc}1 & 0 \\ 0 & \lambda\end{array}\right),\left(\begin{array}{cc}1 & \alpha \\ 0 & 1\end{array}\right)$ et $\left(\begin{array}{ll}1 & 0 \\ \alpha & 1\end{array}\right)$, on montre facilement que l'on peut supposer que $\left(\begin{array}{ll}a_{i} & c_{i} \\ c_{i} & b_{i}\end{array}\right)=\left(\begin{array}{ll}0 & 1 \\ 1 & 0\end{array}\right)$ ou $\left(\begin{array}{ll}2 & 1 \\ 1 & 2\end{array}\right)$.

Or, sur $\mathbb{t}_{2}$ on a la relation:

$$
\left(\begin{array}{ll}
2 & 1 \\
1 & 2
\end{array}\right) \boxplus\left(\begin{array}{ll}
2 & 1 \\
1 & 2
\end{array}\right) \simeq\left(\begin{array}{ll}
0 & 1 \\
1 & 0
\end{array}\right) \boxplus\left(\begin{array}{ll}
0 & 1 \\
1 & 0
\end{array}\right) \text { (théorème 1.41.) }
$$

done a fortiori cela est vrai sur $2 / 8,2=\cdots / 8$. Finalement, pour une raison de déterminant, on peut supposer que

$$
\left(\begin{array}{ll}
a_{i} & c_{i} \\
c_{i} & b_{i}
\end{array}\right)=\left(\begin{array}{ll}
0 & 1 \\
1 & 0
\end{array}\right) \quad \forall i
$$

Résumons-nous. On a donc montré que :

$$
<1>\boxplus\left(\begin{array}{ll}
0 & 1 \\
1 & 0
\end{array}\right) \boxplus \cdots \boxplus\left(\begin{array}{ll}
0 & 1 \\
1 & 0
\end{array}\right) \simeq<1>\boxplus \cdots \boxplus<1>
$$


Mais cela n'est pas vrai. Ln effet, on a $<1>\boxplus\left(\begin{array}{ll}0 & 1 \\ 1 & 0\end{array}\right) \simeq<1>\boxplus<1>\boxplus<-1>$. (la matrice de changement de base est $\left(\begin{array}{ccc}1 & 1 & 1 \\ 1 & 0 & 1 \\ 0 & -1 & -1\end{array}\right)$ ). Donc, on a

$$
<1>\boxplus \cdots \boxplus<1>\simeq \underbrace{\leq 1>\boxplus \cdots \otimes 1>}_{(n+1) / 2} \boxplus \underbrace{<-1>\boxplus \cdots \boxplus<-1>}_{(n-1) / 2} .
$$

Le lemme 4.9 nous permet donc de dire que :

$$
<1>\boxplus \cdots \boxplus<1>\simeq-(\underbrace{<1>\boxplus \cdots \otimes 1>}_{(n-1) / 2})
$$

On en déduit donc que $t_{1}((n-1) / 2)=t_{7}((n-1) / 2)$, mais cela est faux si $n \not \equiv 1 \quad(\bmod 8)$ vu la proposition 4.5. On a donc une contradiction.

\section{Lemme 4.11}

Soit $(V, \beta)$ tel que $M_{\beta}=I_{n}$ et $n \equiv 1 \quad(\bmod 8)$. Posons $x=\left(a_{1}, \ldots, a_{n}\right)$ tel que $\beta(x, x)=1$. Alors, $x$ possède un supplérnentaire orthogonal pair si ct seulement si $a_{1}, \ldots, a_{n}$ sont tous impairs.

\section{Démonstration :}

“” :

$$
\begin{gathered}
\text { Posons } M=\left(\begin{array}{cccccc}
a_{1} & a_{2} & a_{3} & \ldots & a_{n-1} & a_{n} \\
a_{1} & 0 & a_{3} & \ldots & a_{n-1} & a_{n} \\
a_{1} & a_{2} & 0 & \ldots & a_{n-1} & a_{n} \\
\vdots & \vdots & & \ddots & \vdots & \vdots \\
a_{1} & a_{2} & a_{3} & \ldots & 0 & a_{n} \\
a_{1} & a_{2} & a_{3} & \ldots & a_{n-1} & 0
\end{array}\right) . \\
\text { On a donc: } M \cdot M^{L}=\left(\begin{array}{cccccc}
1 & 0 & 0 & 0 & \ldots & 0 \\
0 & 0 & -1 & -1 & \ldots & -1 \\
0 & -1 & 0 & -1 & \ldots & -1 \\
0 & -1 & -1 & \ddots & & \vdots \\
\vdots & \vdots & \vdots & & \ddots & -1 \\
0 & -1 & -1 & \ldots & -1 & 0
\end{array}\right)=<1>\boxplus A \text {. }
\end{gathered}
$$

$A$ est du type $I_{8 k}-J_{8 k}$ où $J_{8 k}=\left(\begin{array}{ccc}1 & \ldots & 1 \\ \vdots & & \vdots \\ 1 & \ldots & 1\end{array}\right)$. Bien sûr, $J_{8 k}^{2} \equiv 0 \quad(\bmod 8)$, donc $A \cdot\left(I_{8 k}+J_{8 k}\right)=I_{8 k}$. Ce qui veut dire que $A$, donc $M$, est inversible. Et trivialement $A$ ne représente que des éléments pairs,

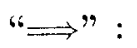

Montrons la contraposée: : sans limiter la généralité, on peut supposer que $a_{n}$ est pair. Comme $\beta(x, x)=1$, et vu le lemme 1.6, on a $V=\langle x\rangle \boxplus\langle x\rangle$. De plus, $a_{1}^{2}+\cdots+a_{n}^{2} \quad$ । $\equiv 1$ ou $5 \quad(\bmod 8)$ donc $\left(a_{1}, \ldots, a_{n-1}\right)$ possède un supplémentaire orthogonal $l$ de dimension impaire pour la forme $\beta^{\prime}$ définie par la matrice $I_{n-1}$. Alors, il existe $y^{\prime}=\left(b_{1}, \ldots, b_{n} 1\right) \in U$ tel que $\beta^{\prime}(y, y)$ soit impair. Finalement, $y=\left(b_{1}, \ldots, b_{n-1}, 0\right)$ appartient à $\langle x\rangle^{-}$, et $\beta(y, y)$ est impair.

\section{Remarque :}

Il $\mathrm{y}$ a donc $4^{n}$ "mauvais vecteurs" si $n \equiv 1 \quad(\bmod 8)$. 


\section{Théorème 4.12}

Si $V=(\omega / 8 \ldots)^{n}$ et $\beta$ cost la lorme bilinéaire définie par la matrice $I_{n}$, alors :

$$
\left|O_{3}^{n}(\cdots / 8)\right|=\prod_{k=1}^{n} t^{\prime}{ }_{1}(k)
$$

$$
\text { où } t^{\prime}{ }_{1}(k)=\left\{\begin{array}{lll}
t_{1}(k) & \text { si } k \neq \equiv 1 & (\bmod 8) \text { ou } k=1 \\
t_{1}(k)-1^{k}=2^{3 k \cdot 3}\left(1-2^{(1 \cdots k) / 2}\right)\left(1+2^{(3-k) / 2}\right) & \text { si } k \equiv 1 & (\bmod 8) \text { et } k \neq 1
\end{array}\right.
$$

\section{Démonstration :}

Par récurrence sur $n$ : si $n=1$, c'est clair.

Supposons que $n \neq \equiv 1 \quad(\bmod 8)$, et soit $x \in V$ tel que $\beta(x, x)=1$. On sait que $\langle x\rangle \boxplus\langle x\rangle^{+} \simeq(\mathbb{Z} / 8 \mathbb{Z})^{n}$, donc vu les lemmes 4.9 et $4.10,\langle x\rangle-\simeq(0 / 8)^{n \cdots 1}$. On conclut alors par récurrence, en raisonnant sur $\langle x\rangle^{\perp}$.

Si $n \equiv 1 \quad(\bmod 8)$, on prend $x$ tel que $\beta(x, x)=1, x$ étant un "bon vecteur". Alors, en faisant le même raisonnement, et en utilisant le lemme 4.11 , on conclut.

\section{Remarque :}

Cette formule est en contradiction avec celle citéc darıs [5, ch. $V, \$ 25, p p$ 186-187], mais nous avons toutes les raisons de croire que la notre est la bonne. En particulier parce que la formule de Minkowski-Siegel que nous obtenons lors du chapit re suivant correpond à celle citée dans [4, ch. 16, §2]. 


\section{CHAPITRE 5}

\section{Calcul explicite de la formule de Minkowski-Siegel pour les formes entières et définies positives.}

Pour ce chapitre, on se fixe $(M, \beta) \in \mathscr{F}_{n}, V=M \otimes \mathbb{Q}, \mu_{p}$ une mesure sur $O\left(V_{p}\right)$ pour tout $p \in \mathbb{F}$, normée de telle manière que $c_{\mu, p}=\frac{1}{2}$, et $M_{1}=M, M_{2}, \ldots, M_{k}$, des représentants de chaque classe d'équivalence dans $\mathscr{G}_{n}$, le genre de $M$.

\section{Théorème 5.1}

Rappelons que $m\left(O(V) \backslash^{\widetilde{O}(V)}\right)$ représente la mesure d'un domaine fondamental pour $\widetilde{O}(V)$ relatif à $O(V)$. Cette constante ne dépend que de $n$, nous l'appellerons $c(n)$. De plus, on a :

$$
c(0)=1, c(1)=\frac{1}{2}, c(2)=\frac{1}{2 \pi} \text { el cc(n)= } \frac{c(n-1)}{n \cdot \rho_{n}} \text { ò̀ } \rho_{n} \text { est le volume de la boule } B^{n}, n \geq 3
$$

\section{Dérnonstration :}

Ce résultat, qui demande une bonne, vingtaine de pages de preuve, est démontré dans [7, ch. $X, \S 34$.]. Il est aussi prouvé dans [5, Satz 26.1] avec les recommandations d'usage concernant cet ouvrage. Enfin, il est démontré dans l'article originel de Siegel [11, Hilfsatz $26+\S 9$ et $\S 10]$.

\section{Corollaire 5.2}

$$
c(n)= \begin{cases}\pi^{\frac{1-n^{2}}{4}} \cdot 2^{\frac{n^{2}-8 n \cdots 9}{8}} \cdot \prod_{i=1}^{\frac{n-1}{2}} 1 \cdot 3 \cdots(2 i-1) \cdot(i-1) ! & \text { si } n \text { est impair } \\ \pi^{\frac{-n^{2}}{4}} \cdot 2^{\frac{-n^{2}-6 n-8}{8}} \cdot\left(\frac{n-2}{2}\right) ! \cdot \prod_{i=1}^{\frac{n-2}{2}} 1 \cdot 3 \cdots(2 i-1) \cdot(i-1) ! & \text { si } n \text { est pair. }\end{cases}
$$

\section{Démonstration :}

On rappelle que

$$
\rho_{n}=\frac{\pi^{\frac{n}{2}}}{\Gamma\left(\frac{n}{2}+1\right)},
$$

ò̀ $\Gamma$ est définie par $\Gamma^{\top}(z)=\int_{0}^{\infty} t^{z} t_{\ell}{ }^{t} d t$. Coute fonction est définie sur les nombres complexes de partie réelle strictement positive. On a :

$$
\begin{aligned}
c(n) & =\frac{c(n-1) \Gamma\left(\frac{n}{2}+1\right)}{n \cdot \pi^{\frac{n}{2}}}=\cdots=\frac{\pi^{\frac{1}{2}} \cdot \prod_{i=3}^{n} \Gamma\left(\frac{i}{2}+1\right)}{n ! \cdot \pi^{\frac{n}{2}} \cdots \cdots \pi^{\frac{1}{2}}} \\
& =\frac{2 \cdot \prod_{i=1}^{n} \Gamma\left(\frac{i}{2}+1\right)}{n ! \cdot \pi^{\frac{n(n+1)}{4}}} .
\end{aligned}
$$

La dernière égalité utilise le lait que $\Gamma\left(\frac{3}{2}\right)=\frac{1}{2} \cdot \pi^{\frac{1}{2}}$ ct que $\Gamma^{\urcorner}(2)=1$. Ces propriétés de la fonction $\Gamma$ ainsi que toutes celles que j'utiliserai par la suite sont citées dans [1, ch. 6].

Comme autre propriété, on a que $\Gamma(z+1)=z \Gamma(z) \forall z$, donc

$$
c(n)=\frac{\prod_{i=1}^{n} \Gamma\left(\frac{i}{2}\right)}{2^{n} 1 \cdot \pi^{\frac{n(n+1)}{4}}} .
$$


La fonction $\Gamma$ est telle que :

$$
\Gamma(m+1)=m ! \quad \text { et } \quad \Gamma\left(m+\frac{1}{2}\right)=\frac{1 \cdot 3 \cdots(2 m-1)}{2^{m}} \pi^{\frac{1}{2}} \quad \text { pour tout } m \in \mathrm{N} .
$$

Supposons que $n$ soit impair. On a:

$$
\prod_{i=2,4, \ldots, n-1} \Gamma\left(\frac{i}{2}\right)=\prod_{i=1}^{\frac{n-1}{2}} \Gamma(i)=\prod_{i=1}^{\frac{n-1}{2}}(i-1) !
$$

Or a aussi :

$$
\begin{aligned}
\prod_{i=1,3, \ldots, n} \Gamma\left(\frac{i}{2}\right) & =\prod_{i=0}^{\frac{n+1}{2}} \Gamma\left(i+\frac{1}{2}\right)=\pi^{\frac{1}{2}} \cdot \prod_{i=1}^{\frac{n-1}{2}} \frac{1 \cdot 3 \cdots(2 i-1) \cdot \pi^{\frac{1}{2}}}{2^{i}} \\
& =2^{\frac{1-n^{2}}{8}} \cdot \pi^{\frac{n+1}{4}} \prod_{i=1}^{\frac{n-1}{2}} 1 \cdot 3 \cdots(2 i-1) .
\end{aligned}
$$

D'où :

$$
c(n)=\pi^{\frac{1-n^{2}}{4}} \cdot 2^{\frac{-n^{2}-8 n-9}{8}} \cdot \prod_{i=1}^{\frac{n-1}{2}} 1 \cdot 3 \cdots(2 i-1) \cdot(i-1) !
$$

Si $n$ est pair, on a :

$$
\prod_{i=2,1, \ldots, n} \Gamma\left(\frac{i}{2}\right)=\prod_{i=1}^{\frac{n}{2}}(i-1) !
$$

et

$$
\begin{aligned}
\prod_{i=1,3, \ldots, n \rightarrow 1} \Gamma\left(\frac{i}{2}\right) & =\pi^{\frac{1}{2}} \cdot \prod_{i=1}^{\frac{n-2}{2}} \frac{1 \cdot 3 \cdots \cdot(2 i-1) \cdot \pi^{\frac{1}{2}}}{2^{i}} \\
& =2^{\frac{(2-n) n}{6}} \cdot \pi^{\frac{n}{4}} \prod_{i=1}^{\frac{n-2}{2}} 1 \cdot 3 \cdots(2 i-1) .
\end{aligned}
$$

Done

$$
c(n)=\pi^{\frac{-n^{2}}{4}} \cdot 2^{-n^{2} \frac{6 n-8}{8}} \cdot\left(\frac{n-2}{2}\right) ! \cdot \prod_{i=1}^{\frac{n-2}{2}} 1 \cdot 3 \cdots(2 i-1) \cdot(i-1) ! .
$$

\section{A. La formule de Minkowski-Siegel dans le cas de $\mathscr{C}_{n}$.}

Supposons que $M$ soit de type $(I I)$. Alors, $n \equiv 0 \bmod 8$. Posons $q(x)=\frac{1}{2} \beta(x, x), M$ est un module quadratique non dégénéré pour cette forme. On a donc $M_{p}=M_{p}^{\#}$, pour tout $p \in \mathbb{E}$. On obtient alors grâce au corollaire 2.49, au théorème 2.59 , ainsi qu'au théorème 3.8 et au corollaire 3.9 :

$$
\mathscr{M}_{\mathscr{Q}_{n}}=c(n) \cdot \prod_{p C}\left(1-p^{-\frac{n}{2}}\right)^{-1} \cdot \prod_{p \in \mathbb{E}} \prod_{0<2 i<n}\left(1-p^{2 i}\right)^{-1}
$$

Ceci, parce que le discriminant de $M$ valul 1.

\section{Théorème 5.3}

On a :

$$
\mathscr{H}_{\mathscr{S}_{\pi}}=2^{1-n} \cdot \frac{\left|B_{\frac{n}{2}}\right|}{\left(\frac{n}{2}\right) !} \cdot \prod_{i=1}^{\frac{n}{2}-1}\left|B_{2 i}\right|
$$

où $B_{i}$ sont les nombres de Bernoulli. Ces nombres sont définis darns [1, ch. 23] par exemple. 


\section{Démonstration :}

Nous avons tout d'abord besoin d'un résultat classique sur la fonction $\zeta$ de Riemann :

$$
\zeta(2 i)=\prod_{p \in \mathbb{P}}\left(1-p^{-2 i}\right)^{-1}=\frac{(2 \pi)^{2 i}}{2(2 i) !}\left|B_{2 i}\right| .
$$

Ce résultat est démontré dans [10, ch. VII, Proposition 7]. On trouve alors:

$$
\mathscr{M}_{\mathscr{B}_{n}}=\pi^{\frac{-n^{2}}{4}} \cdot 2^{-n^{2} 6 n+8} \cdot\left(\frac{n-2}{2}\right) ! \cdot \prod_{i}^{\frac{n-2}{2}} 1 \cdot 3 \cdots(2 i-1) \cdot(i-1) ! \cdot \frac{(2 \pi)^{\frac{n}{2}}}{2} \cdot \prod_{0<2 i<n} \frac{(2 \pi)^{2 i}}{2(2 i) !} \cdot K
$$

où $K=\frac{\left|B_{\frac{n}{2}}\right|}{\left(\frac{n}{2}\right) !} \cdot \prod_{i=1}^{\frac{n}{2}-1}\left|B_{2 i}\right|$. En développant, on trouve :

$$
\begin{aligned}
\mathscr{M}_{\mathscr{Q}_{n}} & =\pi^{\frac{-n^{2}+2 n}{4}} \cdot \pi^{\frac{n^{2}-2 n}{4}} \cdot 2^{\frac{-n^{2}-6 n}{8}} \cdot 2^{\frac{n}{2}} \cdot 2^{\frac{-n+2}{2}} \cdot 2^{\frac{n^{2}-2 n}{4}} \cdot\left(\frac{n-2}{2}\right) ! \cdot \prod_{i=1}^{\frac{n-2}{2}} \frac{1 \cdot 3 \cdots(2 i-1)(i-1) !}{(2 i) !} \cdot K \\
& =2^{\frac{n^{2}-10 n+8}{8}} \cdot\left(\frac{n-2}{2}\right) ! \cdot \prod_{i=1}^{\frac{n-2}{2}} \frac{(i-1) !}{2^{2} \cdot i !} \cdot K \\
& =2^{\frac{n^{2}-10 n+8}{8}} \cdot \prod_{i=1}^{\frac{n+2}{2}} \frac{1}{2^{i}} \cdot K \\
& =2^{1-n} \cdot K .
\end{aligned}
$$

B. La formule de Minkowski-Siegel dans le cas de $\mathscr{C}_{n}$.

Supposons maintenant que $M$ soit de lype (I). Puisque la formule ne dépend que du genre, on peut supposer que $\beta$ soil ha forme $x_{1} y_{1}+\cdots+x_{n} y_{n}$. Posons $q(x)=\beta(x, x)$. Celle fois-ci, $(M, q)$ est un module quadratique dégénéré. On a néanmoins $M_{p}=M_{p}^{\text {\# }}$ pour 1 out $p$ différent de 2 . Pour $p=2$, on trouve $M_{2}=2 M_{2}^{H}$. Le but est de calculer $\mu_{2}\left(O\left(M_{2}\right)\right.$ ). Gráce au théoreme 2.57, nous savons que $c_{\mu, 2}$ est constant si $k=4$ par excmple. On a :

$$
\mu_{2}\left(O\left(M_{2}\right)\right)=\left[O\left(M_{2}\right): O\left(V_{2}, M_{2} / 2^{4} M_{2}^{\#}\right)\right] \cdot \mu_{2}\left(O\left(V_{2}, M_{2} / 2^{4} M_{2}^{\#}\right)\right)
$$

On trouve alors, par la définition de $c_{\mu .2}$ :

$$
\mu_{2}\left(O\left(V_{2}, M_{2} / 2^{4} M_{2}^{1 t}\right)\right)=c_{\mu, 2} \cdot\left[M_{2}: 2^{3} M_{2}\right]^{\frac{1-n}{2}}=c_{\mu, 2} \cdot 2^{\frac{3 n(1 \cdots n)}{2}}
$$

\section{Proposition 5.4}

Posons $\overline{M_{2}}=M_{2} / 2^{3} M_{2}$ vu comme $\cdots / 8 \ldots$ module. Alors :

$$
\left[O\left(M_{2}\right): O\left(V_{2}, M_{2} / 2^{3} M_{2}\right)\right]=\left|O_{\beta}^{n}\left(\overline{M_{2}}\right)\right| \cdot \frac{1}{2^{n}}=\left|O_{\beta}^{n}(\omega / 82)\right| \cdot \frac{1}{2^{n}}
$$

Le groupe $O_{\beta}^{n}(\mathbb{Z} / 8 \mathbb{Z})$ à été dófini au chapitre 4 .

\section{Dérnonstration :}

L'application :

$$
\begin{aligned}
\psi: O\left(M_{2}\right) & \longrightarrow O_{\beta}^{r}(\cdots / 8) \\
u & \longmapsto \bar{u}: \overline{M_{2}} \longrightarrow \overline{M_{2}} \\
& x+2^{3} M_{2} \longmapsto u(x)+2^{3} M_{2}
\end{aligned}
$$


est bien définie *. Son noyau est bien $O\left(V_{2}, M_{2} / 2^{3} M_{2}\right)$. Mais on ne peut pas utiliser le lemme 2.52, donc $\psi$ n'est pas forcément surjective. On va montrer que $\left[O_{\beta}^{n}(Z / 8): \operatorname{Im}(\psi)\right]=2^{\mathrm{n}}$.

Soit $\bar{u} \in O_{\beta}^{n}(-/ 8 \bar{z}), \bar{u}$ peut otre représentée par une matrice $A \in M_{n}\left(\Xi_{2}\right)$ telle que $A \cdot A^{t}=I_{n}+8 \cdot B$ où $B$ est une matrice symétrique. Soit.

$$
\begin{aligned}
\varphi: O_{\beta}^{n}(\mathbb{Z} / 8 & \longrightarrow\left(\mathbb{K}_{2}\right)^{n} \\
A & \longmapsto \operatorname{diag}(B) \quad(\bmod 2) \quad \text { avec } B \text { telle que } 8 B=A A^{t}-I_{n} .
\end{aligned}
$$

\section{Lemme}

$\varphi$ est bien définie, en outre, $\varphi(A)=(0, \ldots, 0)$ si et seulement si $A \in \operatorname{Im}(\psi)$

\section{Démonstration du lemme}

Soit $A \in O_{3}^{n}(\because / 8)$. Supposons que $A_{1}$ et $A_{2} \in M_{n}\left(\omega_{2}\right)$ sont telles que $\overline{A_{1}}=\overline{A_{2}}=A$. Il existe $C \in M_{n}\left(\mathbb{Z}_{2}\right)$ telle que $A_{1}=A_{2}+8 C$. On a :

$$
I_{n}+8 B_{1}=A_{1} A_{1}^{t}=\left(A_{2}+8 C\right)\left(A_{2}^{t}+8 C^{t}\right)=I_{n}+8\left(B_{2}+A_{2} C^{t}+C A_{2}^{t}+8 C C^{t}\right) .
$$

Et on remarque que

$$
\operatorname{diag}\left(B_{1}\right) \equiv \operatorname{diag}\left(B_{2}+A_{2} C^{t}+C A_{2}^{t}+8 C C^{t}\right) \equiv \operatorname{diag}\left(B_{2}\right) \quad(\bmod 2)
$$

car $\operatorname{diag}\left(X+X^{t}\right) \equiv 0 \bmod 2 \forall X \in M_{n}\left(Z_{2}\right)$. Donc $\varphi$ est bien définie.

Soit $\bar{A} \in \operatorname{Im}(w)$; il existe en particulicr $X$ et $B$ telles que

$$
(A+8 X) \cdot(A+8 X)^{t}=I_{n}+8\left(B+A X^{t}+X A^{t}\right) \equiv I_{n} \quad(\bmod 16) .
$$

Donc $B \equiv\left(A X^{t}+X A^{t}\right) \bmod 2$, ce qui veut dire que $\operatorname{diag}(B) \equiv(0, \ldots, 0) \bmod 2$.

Réciproquement, soit $A A^{t}=I_{n}+8 B$, avec $B$ n'ayant que des éléments pairs dans la diagonale. Posons $B^{-}$, la matrice triangulaire inférieure telle que $B^{--}+B{ }^{t}=B$. On a :

$$
\left(A-8 B^{\prime} A\right)(A-8 B \cdot A)^{t} \equiv I_{n} \quad(\bmod 16)
$$

et $A_{1}:=(A-8 B \quad A) \equiv A \bmod 8$. On refait le meme raisomement, on a $A_{1} A_{1}^{t}=I_{n}+16 B_{1}$. On vérifie que $\operatorname{diag}\left(B_{1}\right) \equiv(0, \ldots, 0) \bmod 2$, et on obtient $A_{2}=A_{1}+16 C_{1} \in M_{n}\left(\%_{2}\right)$ tolle que $A_{2} A_{2}^{t} \equiv I_{n}$ mod 32 . Et ainsi de suite; on trouve alors une suite $\left(A_{n}\right)_{n \in n}$ qui converge dans $M_{n}\left(\mathbb{Z}_{2}\right)$. La limite de cette suite est une rnatrice $X$ de $O\left(M_{2}\right)$, et. on a $X \equiv A \bmod 8$. Donc $\bar{A} \in \operatorname{Im}(\psi)$.

Ainsi s'achève la démonstration du lemme.

Notre application $\varphi$ est surjective. En effet, soit $B \in M_{n}\left(\Theta_{2}\right)$ symétrique. La somme

$$
I_{n}+\frac{1}{2} \cdot(8 B)-\frac{1}{2^{2} \cdot 2} \cdot(8 B)^{2}+\frac{1 \cdot 3}{2^{3} \cdot 2 \cdot 3} \cdot(8 B)^{3}+\cdots+(-1)^{n-1} \cdot \frac{1 \cdot 3 \cdots \cdot(2 n-3)}{2^{n} \cdot n !} \cdot(8 B)^{n}+\cdots
$$

converge. En effet, dans $n !$ nous avons au plus $\left[\frac{n}{2}\right]+\left[\frac{n}{4}\right]+\cdots$ facteurs 2 . Or,

$$
\left[\frac{n}{2}\right]+\left[\frac{n}{4}\right]+\cdots \leq \frac{n}{2}+\frac{n}{4}+\cdots=n \cdot\left(\frac{1}{2}+\frac{1}{4}+\cdots\right)=n .
$$

La norme $p$-adique du $n$-ìme terme de notre série est donc au plus $2^{--n}$, elle est alors convergente. Notons $A$ sa limite, qui est une matrice symétrique puisque $B$ l'est.

\footnotetext{
* Remarquons que $\psi$ aurait ćté bien définic aussi, si nous avions pris $O_{q}^{n}(\ldots / 8 \ldots)$ comme groupe d'arrivéc. Nous prenons alors le plus petit des deux (voir ie premier paragraphe du chapitre 4)
} 
Tout le monde aura reconmu dans cote somme, le développement de Taylor de $\sqrt{I_{n}+8 B}$. Donc, on a :

$$
A^{2}=A \cdot A^{l}=I_{n}+8 B
$$

$\varphi$ est donc surjective, il suflit de prendre $B$ avec une diagonale convenable.

Il nous reste à voir que $A_{1}^{-1} A_{2} \in \operatorname{Im}(\psi)$ si et seulement si $\varphi\left(A_{1}\right)=\varphi\left(A_{2}\right)$.

Supposons que $A_{1}^{-1} A_{2} \in \operatorname{Im}(\psi)$. On a $\left(A_{1}^{-1} A_{2}\right)\left(A_{1}^{-1} A_{2}\right)^{t}=I_{n}+8 B$ avec $B$ symétrique ne comportant que des éléments pairs dans la diagonale. Calculons :

$$
\left(A_{1}^{-1} A_{2}\right)\left(A_{1}^{-1} A_{2}\right)^{t}=A_{1}^{-1}\left(I_{n}+8 B_{2}\right) A_{1}^{t^{-1}}
$$

On vérifie que $A_{1}^{-1} A_{1}^{t^{-1}}=I_{n}-8 A_{1}^{-1} B_{1} A_{1}^{t^{-1}}$. On trouve finalement :

$$
I_{n}+8 B=I_{n}+8 A_{1}^{-1}\left(B_{2}-B_{1}\right) A_{1}^{t^{-1}}
$$

Autrement dit, $A_{1}^{-1}\left(B_{2}-B_{1}\right) A_{1}^{t^{-1}} \equiv B \bmod 2 ; \operatorname{donc} \operatorname{diag}\left(B_{2}-B_{1}\right) \equiv 0 \operatorname{mrod} 2$, ce qui veut dire que $\varphi\left(A_{1}\right)=\varphi\left(A_{2}\right)$.

On démontre la réciproque de manière identique.

\section{Lemme 5.5}

Supposons que $n$ soit impair, alors on a :

$$
c(n) \cdot \prod_{0<2 i<n} \prod_{p \in ?^{2}}\left(1-p^{2 i}\right)^{i}=2^{\frac{n+1}{2}} \cdot 2^{\cdots 2 n+2} \cdot \frac{1}{\left(\frac{n-1}{2}\right) !} \cdot\left|B_{2} \cdots B_{n-1}\right| .
$$

\section{Démonstration :}

Le corollaire 5.2 nous permet de dire :

$$
\begin{aligned}
& c(n) \cdot \prod_{0<2 i<n} \prod_{p \in \mathbb{R}}\left(1-p^{-2 i}\right)^{1}=\pi^{\frac{1 \cdots n^{2}}{4}} \cdot 2^{\frac{-n^{2}-8 n+9}{8}} \cdot \prod_{i=1}^{\frac{n \cdots 1}{2}} 1 \cdot 3 \cdots \cdots(2 i-1) \cdot(i-1) ! \cdot \prod_{i=2,4, \ldots, n-1} \frac{\left|B_{i}\right| \cdot(2 \pi)^{i}}{2 \cdot(i) !} \\
& =\pi^{\frac{1 \ldots n^{2}}{4}} \cdot 2^{\frac{-n^{2}-8 n+9}{8}} \cdot(2 \pi)^{\frac{2\left(n^{2}-1\right)}{8}} \cdot \prod_{i=1}^{\frac{n-1}{2}} \frac{1 \cdot 3 \cdots(2 i-1)}{(2 i) !} \cdot \frac{1}{2} \cdot(i-1) ! \cdot \prod_{i=1}^{\frac{n-1}{2}}\left|B_{2 i}\right|
\end{aligned}
$$

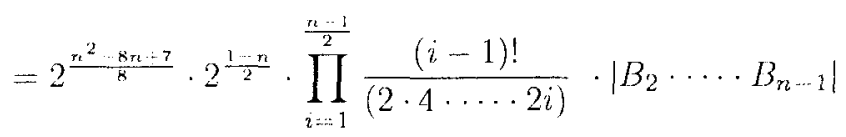

$$
\begin{aligned}
& =2^{\frac{n^{2}-12 n+11}{8}} \cdot 2^{\frac{1 \cdots n^{2}}{8}} \cdot \prod_{i=1}^{\frac{n-1}{2}} \frac{1}{i} \\
& =2^{\frac{n-1}{2}} \cdot 2^{2 n+2} \cdot \frac{1}{\left(\frac{n}{2}\right) !} \cdot\left|B_{2} \cdots \cdot B_{n} \quad 1\right| \text {. }
\end{aligned}
$$

\section{Lemme 5.6}

Posons $\kappa(s)=\sum_{k=1}^{\infty}(-1)^{k}(2 k+1)^{-s}$. Alors, pour tout $m \in \amalg$, on a

$$
\kappa(2 m+1)=\prod_{p \in \mathbb{P} \backslash\{2\}}\left(1-(-1)^{\frac{p-1}{2}} \cdot p^{-(2 m+1)}\right)=\frac{(\pi / 2)^{2 m+1}}{2(2 m) !}\left|E_{2 m}\right|
$$

Où $E_{i}$ sont les nombres diEuler. Ces nombres sont définis dans [1, ch. 23] par exemple. 


\section{Démonstration :}

La deuxième égalité est citéo dans $[1,23.2 .22]$. D'autre part, on a

$$
\kappa(s)=\sum_{n=1}^{\infty} \frac{c_{n}}{n^{s}} .
$$

On vérifie sans peine que les $c_{n}$ sont multiplicatifs, donc

$$
\kappa(s)=\prod_{p \in \mathbb{Z}} \sum_{m=0}^{\infty} c_{p^{m}} p^{\cdots \cdots}
$$

Or

$$
c_{p^{m}}=\left\{\begin{array}{lll}
1 & \text { si } p \equiv 1 & (\bmod 4) \\
(-1)^{m} & \text { si } p \equiv 3 \\
0 & \text { si } p=2 & (\bmod 4)
\end{array}\right.
$$

On trouve alors $c_{p^{m}}=(-1)^{\frac{(p \cdot 1) m}{2}}$ si $p$ est impair. Pour un tel $p$, on a :

$$
\sum_{m=0}^{\infty}\left((-1)^{\frac{p-1}{2}} \cdot p^{-s}\right)^{m}=\left(1-(-1)^{\frac{p--1}{2}} \cdot p^{-s}\right)
$$

par propriété des séries géométriques. On a donc bien :

$$
\kappa(s)=\prod_{m,\{2\}}\left(1-(-1)^{\frac{p-1}{2}} \cdot p^{\cdot(2 m+1)}\right) .
$$

\section{Lemme 5.7}

Supposons que, $n \equiv \pm 2 \bmod 8$, alors on a :

$$
c(n) \cdot \prod_{0<2 i<n}\left(1-p^{2 i}\right)^{-1} \cdot \prod_{p \in \backslash\{2\}}\left(1-(-1)^{\frac{p-1}{2}} \cdot p^{-\frac{n}{2}}=\frac{2^{-2 n-1}}{\left(\frac{n-2}{2}\right) !} \cdot\left|E_{\frac{n-2}{2}} \cdot B_{2} \cdots \cdot B_{n-2}\right|\right.
$$

\section{Démonstration :}

$$
\begin{aligned}
& c(n) \cdot \prod_{0<2 i<n}\left(1-p^{-2 i}\right)^{-1} \cdot \prod_{p \in \backslash\{2\}}\left(1-(-1)^{\frac{p-1}{2}} \cdot p^{-\frac{n}{2}}\right)=\pi^{\frac{-n^{2}}{4}} \cdot 2^{-\frac{n^{2}-6 i n+8}{8}} \cdot\left(\frac{n-2}{2}\right) ! \cdot \frac{\left(\frac{\pi}{2}\right)^{\frac{n}{2}}}{2\left(\frac{n-2}{2}\right) !} \cdot\left|E_{\frac{n-2}{2}}\right| \\
& =\cdot \prod_{i=1}^{\frac{n \cdots 2}{2}} 1 \cdot 3 \cdots(2 i-1) \cdot(i-1) ! \cdot \prod_{i=1}^{\frac{n-2}{2}} \frac{(2 \pi)^{2 i}}{2(2 i) !}\left|B_{2 i}\right| \\
& =\cdots \\
& =\frac{2^{-\cdots 2 n+1}}{\left(\frac{n-2}{2}\right) !} \cdot\left|E_{\frac{n-2}{2}} \cdot B_{2} \cdots \cdot B_{n-2}\right| \text {. }
\end{aligned}
$$

Nous voilà enfin prêt à démontrer lo théorème principal de ce diplôme. 


\section{Théorème 5.8}

$$
\mathscr{M}_{\mathscr{C}_{n}}=\left\{\begin{array}{lr}
\frac{\left(1+2^{\frac{2-n}{2}}\right)\left(1-2^{\frac{-n}{2}}\right)}{2 \cdot\left(\frac{n}{2}\right) !} \cdot\left|B_{\frac{n}{2}} \cdot B_{2} \cdots B_{n-2}\right| & \text { si } n \equiv 0 \quad(\bmod 8) \\
\frac{\left(2^{\frac{n-1}{2}}+1\right)}{2^{n} \cdot\left(\frac{n-1}{2}\right) !} \cdot\left|B_{2} \cdots B_{n-1}\right| & \text { si } n \equiv \pm 1 \quad(\bmod 8) \\
\frac{1}{2^{n+1} \cdot\left(\frac{n-2}{2}\right) !} \cdot\left|E_{\frac{n-2}{2}} \cdot B_{2} \cdots B_{n-2}\right| & \text { si } n \equiv \pm 2 \quad(\bmod 8) \\
\frac{\left(2^{\frac{n-1}{2}}-1\right)}{2^{n} \cdot\left(\frac{n-1}{2}\right) !} \cdot\left|B_{2} \cdots \cdot B_{n-1}\right| & \text { si } n \equiv \pm 3 \quad(\bmod 8) \\
\frac{\left(1-2^{\frac{2-n}{2}}\right)\left(1-2^{\frac{-n}{2}}\right)}{2 \cdot\left(\frac{n}{2}\right) !} \cdot\left|B_{\frac{n}{2}} \cdot B_{2} \cdots B_{n-2}\right| & \text { si } n \equiv 4 \quad(\bmod 8) .
\end{array}\right.
$$

\section{Démonstration :}

Vu la proposition 5.4 et le théorème 4.12 , on a :

$$
\begin{aligned}
& \mu_{2}\left(O\left(M_{2}\right)\right)=c_{\mu, 2} \cdot 2^{\frac{3 n(1 \cdots n)}{2}} \cdot 2^{\cdots n} \cdot\left|O_{\beta}^{n}(-/ 8 Z)\right| \\
& =2^{n-1 \cdot 1} \cdot 2^{\frac{3 n(3-n)}{2}} \prod_{k=1}^{n} i_{1}^{\prime}(k) .
\end{aligned}
$$

a) Supposons que $n=8$. On a, grâce au théorime 4.12 et à la proposition 4.4 :

$$
\begin{aligned}
\mu_{2}\left(O\left(M_{2}\right)\right) & =2^{-9} \cdot 2^{-81} \cdot 2^{3-6+\cdots 221} \cdot 8 \cdot \frac{1}{\left(1+2^{-3}\right)\left(1-2^{-4}\right)} \cdot\left(1-2^{-2}\right)\left(1-2^{-4}\right)\left(1-2^{-6}\right)\left(1-2^{-4}\right) \\
& =2^{-6} \cdot \frac{1}{\left(1+2^{-3}\right)\left(1-2^{-4}\right)} \cdot\left(1-2^{-2}\right)\left(1-2^{-1}\right)\left(1-2^{-6}\right)\left(1-2^{-4}\right) .
\end{aligned}
$$

Si $p \neq 2$, on trouve comme pour le type (II)

$$
\mu_{p}\left(O\left(M_{p}\right)\right)=\left(1-p^{4}\right) \cdot \prod_{0<2 i<8}\left(1-p^{-2 i}\right) .
$$

On trouve alors

$$
\begin{aligned}
\mathscr{M}_{\mathscr{S}_{8}} & =c(8) \cdot \prod_{p}\left(1-p^{4}\right)^{-1} \cdot \prod_{0<2 i<8} \prod_{p \in \mathbb{R}}\left(1-p^{-2 i}\right) \cdot \frac{\left(1+2^{-3}\right)\left(1-2^{-4}\right)}{2^{-6}} \\
& =\frac{\left(1+2^{-3}\right)\left(1-2^{-4}\right)}{2^{7-6} \cdot 4 !} \cdot\left|B_{4} \cdot B_{2} \cdot B_{4} \cdot B_{6}\right| .
\end{aligned}
$$

La dernière égalité vient du calcul fait pour le type $(I I)$.

b) Supposons $n \equiv 0 \bmod 8, n>8$, et que

$$
\left|O_{\beta}^{n-8}\right|=\frac{2^{\frac{3(n-3)(n 9)}{2}} \cdot 8}{\left(1+2^{\frac{10-n}{2}}\right)}\left(1-2^{2}\right)\left(1-2^{-4}\right) \cdots\left(1-2^{10-n}\right) .
$$

Calculons :

$$
\begin{aligned}
& \left|O_{B}^{n}\right|=2^{3 i(n-1) \cdots \cdots \cdot(n-8)}\left(1-2^{\frac{2-n}{2}}\right)\left(1-2^{\frac{4-n}{2}}\right)\left(1+2^{\frac{1-n}{2}}\right)\left(1-2^{\frac{6-n}{2}}\right)\left(1+2^{\frac{6-n}{2}}\right) \\
& \cdot\left(1+2^{\frac{8-n}{2}}\right)\left(1-2^{\frac{8-n}{2}}\right)\left(1+2^{\frac{10-n}{2}}\right) \cdot\left|O_{\beta}^{n-8}\right| \\
& =2^{\frac{3(2 n-9)+3(n-8)(n-9)}{2}} \cdot 8 \cdot\left(1-2^{\frac{2-n}{2}}\right)\left(1-2^{4-n}\right)\left(1-2^{6-n}\right)\left(1-2^{8-n}\right)\left(1+2^{\frac{10-n}{2}}\right) \\
& \cdot \frac{1}{\left(1+2^{\frac{10-n}{2}}\right)} \cdot\left(1-2^{-2}\right)\left(1-2^{-4}\right) \cdots\left(1-2^{10-n}\right) \\
& =\frac{2^{\frac{3 n(n-1)}{2}} \cdot 8}{\left(1+2^{\frac{2 n n}{2}}\right)\left(1-2^{\frac{n}{2}}\right)} \cdot\left(1-2^{-2}\right)\left(1-2^{-4}\right) \cdots\left(1-2^{2-n}\right) \cdot\left(1-2^{-\frac{n}{2}}\right) \text {. }
\end{aligned}
$$


Donc,

$$
\begin{aligned}
\mu_{2}\left(O\left(M_{2}\right)\right) & =c_{\mu, 2} \cdot 2^{-\frac{3 n(n \cdot 1)}{2}} \cdot 2^{n} \cdot\left|O_{3}^{n}(\omega / 8)\right| \\
& =\frac{4 \cdot 2^{-n}}{\left(1+2^{\frac{2-n}{2}}\right)\left(1-2^{\frac{n}{2}}\right)} \cdot\left(1-2^{-2}\right)\left(1-2^{-4}\right) \cdots\left(1-2^{2-n}\right) \cdot\left(1-2^{-\frac{n}{2}}\right) .
\end{aligned}
$$

On trouve alors:

$$
\begin{aligned}
\mathscr{M}_{\mathscr{K}_{n}} & =c(n) \cdot \prod_{p \in \mathbb{R}^{n}}\left(1-p^{-\frac{n}{2}}\right)^{-1} \cdot \prod_{0<2 i<n} \prod_{p \in \mathbb{P}}\left(1-p^{-2 i}\right) \cdot \frac{\left(1+2^{\frac{2-n}{2}}\right)\left(1-2^{-\frac{n}{2}}\right)}{4 \cdot 2^{-n}} \\
& =\frac{\left(1+2^{\frac{2-n}{2}}\right)\left(1-2^{-\frac{n}{2}}\right)}{4 \cdot\left(\frac{n}{2}\right) ! \cdot 2^{-n} \cdot 2^{n-1}} \cdot\left|B_{\frac{n}{2}} \cdot B_{2} \cdots B_{n-2}\right| \\
& =\frac{\left(1+2^{\frac{2-n}{2}}\right)\left(1-2^{-\frac{n}{2}}\right)}{2 \cdot\left(\frac{n}{2}\right) !} \cdot\left|B_{\frac{n}{2}} \cdot B_{2} \cdots B_{n-2}\right| .
\end{aligned}
$$

c) Supposons $n \equiv 1 \bmod 8$.

Si $n=1$, on a clairement que $\mathscr{M}_{o x_{1}}=\frac{1}{2}$. Supposons donc $n \neq 1$. Grâce au calcul précédent, à la proposition 4.2 et au thćorème 4.10 , nous pouvons dire que

$$
\begin{aligned}
& \mu_{2}\left(O\left(M_{2}\right)\right)=2^{n \cdots 1} \cdot 2^{\frac{3 n(n+1)}{2}} \cdot \frac{2^{\frac{3 n(n \cdot n)}{2}} \cdot 8}{\left(1+2^{\frac{3}{2} n^{n}}\right)} \cdot\left(1-2^{n}\right) \cdots\left(1-2^{3 \cdots n}\right)\left(1-2^{\frac{1-n}{2}}\right)\left(1+2^{\frac{3-n}{2}}\right) \\
& =\frac{2^{n-2}}{\left(1+2^{\frac{1}{2}}\right)^{n}}\left(1-2^{n}\right) \cdots\left(1-2^{3}{ }^{n}\right)\left(1-2^{1 n}\right) \text {. }
\end{aligned}
$$

Le lemme 5.5 nous donne: alors :

$$
\begin{aligned}
\mathscr{M}_{\mathscr{H}_{n}} & =\frac{\left(1+2^{\frac{1+n}{2}}\right) \cdot 2^{\frac{n-1}{2}}}{2^{2 n+\cdots 2 \cdots n+2} \cdot\left(\frac{n-1}{2}\right) !} \cdot\left|B_{2} \cdots \cdot B_{n-1}\right| \\
& =\frac{\left(2^{\frac{n-1}{2}}+1\right)}{2^{n} \cdot\left(\frac{n-1}{2}\right) !} \cdot\left|B_{2} \cdots B_{n-1}\right| .
\end{aligned}
$$

d) Supposons $n \equiv 2 \bmod 8$.

Calculons :

$$
\begin{aligned}
\mu_{2}\left(O\left(M_{2}\right)\right) & =c_{\mu .2} \cdot 2^{\frac{\left.3 m_{n} n-1\right)}{2}} \cdot 2^{n} \cdot 2^{\frac{3 n(n-1)}{2}} \cdot \frac{8}{1+2^{\frac{2-n}{2}}} \cdot\left(1-2^{-2}\right)\left(1-2^{-4}\right) \cdots\left(1-2^{2-n}\right)\left(1+2^{\frac{2-n}{2}}\right) \\
& =2^{-n+2} \cdot\left(1-2^{2}\right)\left(1-2^{4}\right) \cdots\left(1-2^{2-n}\right) .
\end{aligned}
$$

Or, le discriminant de $M$ vaut $-2^{n}$; done, si $p$ est impair, le symbole de Legendre $\left(\frac{-2^{n}}{p}\right)=\left(\frac{-1}{p}\right)=(-1)^{\frac{p-1}{2}}$.

On trouve alors:

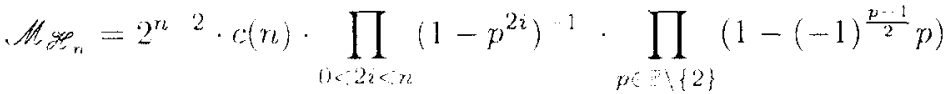

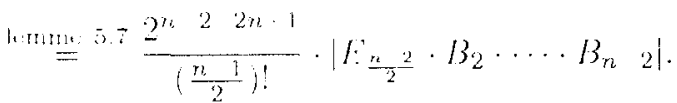

$$
\begin{aligned}
& =\frac{1}{2^{n \cdots 1} \cdot\left(\frac{n+1}{2}\right) !} \cdot\left|E_{\frac{n-2}{2}} \cdot B_{2} \cdots \cdot B_{n \cdot 2}\right| \text {. }
\end{aligned}
$$

e) Supposons $n \equiv 3 \bmod 8$.

On trouve:

$$
\mu_{2}\left(O\left(M_{2}\right)\right)=c_{\mu, 2} \cdot 2^{\cdots n} \cdot\left(1-2^{-2}\right)\left(1-2^{-4}\right) \cdots\left(1-2^{3-n}\right)\left(1-2^{1-n}\right) \cdot \frac{8}{\left(1-2^{\frac{1-n}{2}}\right)} .
$$


D'où

$$
\begin{aligned}
& \mathscr{M}_{\mathscr{W}_{n}}=2^{n-2} \cdot\left(1-2^{\frac{1-n n}{2}}\right) \cdot c(n) \cdot \prod_{0<2 i<n} \prod_{p \in \mathbb{P}}\left(1-p^{\cdots 2 i}\right)^{-1} \\
& \stackrel{\text { lemme }}{=} 5.52^{\frac{2 n-4-4 n+4}{2}} \cdot \frac{\left(2^{\frac{n-1}{2}}-1\right)}{\left(\frac{n-1}{2}\right) !} \cdot\left|B_{2} \cdots B_{n-1}\right| \\
& =\frac{\left(2^{\frac{n-1}{2}}-1\right)}{2^{n} \cdot\left(\frac{n}{2}-1\right) !} \cdot\left|B_{2} \cdots B_{n-1}\right| .
\end{aligned}
$$

f) Supposons $n \equiv 4 \bmod 8$.

On obtient :

$$
\mu_{2}\left(O\left(M_{2}\right)\right)=\frac{2^{-n+2}}{\left(1-2^{\frac{2-n}{2}}\right)\left(1-2^{\frac{-n}{2}}\right)} \cdot\left(1-2^{-2}\right)\left(1-2^{-4}\right) \cdots\left(1-2^{2-n}\right)\left(1-2^{\frac{-n}{2}}\right)
$$

On a alors :

$$
\begin{gathered}
\mathscr{M}_{\mathscr{W}_{n}}=2^{n \cdot 2} \cdot\left(1-2^{\frac{2-n}{2}}\right)\left(1-2^{\frac{-n}{2}}\right) \cdot c(n) \cdot \prod_{p \in \mathbb{R}}\left(1-p^{\frac{-n}{2}}\right) \cdot \prod_{0<2 i<n} \prod_{p \in \mathbb{N}}\left(1-p^{-2 i}\right)^{-1} \\
\stackrel{h_{n} \cdot 3.3}{=} 2^{n-2} n \cdot 1 \cdot \frac{\left(1-2^{\frac{2-n}{2}}\right)\left(1-2^{\frac{-n}{2}}\right)}{\left(\frac{n}{2}\right) !} \cdot\left|B_{\frac{n}{2}} \cdot B_{2} \cdots B_{n-2}\right| .
\end{gathered}
$$

g) Supposons $n \equiv 5 \bmod 8$.

On a :

$$
\begin{aligned}
\mu_{2}\left(O\left(M_{2}\right)\right) & =\frac{2^{-n+2}}{\left(1-2^{\frac{3-n}{2}}\right)} \cdot\left(1-2^{-2}\right)\left(1-2^{-4}\right) \cdots\left(1-2^{3-n}\right) \cdot\left(1-2^{\frac{3-12}{2}}\right) \cdot\left(1+2^{\frac{1-n}{2}}\right) \\
& =\frac{2^{2 n-2}}{\left(1-2^{\frac{1-n}{2}}\right)} \cdot\left(1-2^{2}\right)\left(1-2^{-4}\right) \cdots\left(1-2^{3-n}\right) \cdot\left(1-2^{1-n}\right) .
\end{aligned}
$$

Comme pour $n \equiv 3$ rnod 8 , le lemmo 5.5 nous permet de dire que

$$
\mathscr{M}_{\mathscr{E}_{n}}=\frac{\left(2^{\frac{n^{2}-1}{2}}-1\right)}{2^{n} \cdot\left(\frac{n+1}{2}\right) !} \cdot\left|B_{2} \cdots \cdot B_{n+1}\right| .
$$

h) Supposons $n \equiv 6 \bmod 8$.

On trouve:

$$
\begin{aligned}
\mu_{2}\left(O\left(M_{2}\right)\right) & =\frac{2^{\cdots \cdot 2}}{\left(1-2^{\frac{2 \cdot n}{2}}\right)} \cdot\left(1-2^{-2}\right)\left(1-2^{-4}\right) \cdots\left(1-2^{4 \cdots n}\right) \cdot\left(1-2^{2 \cdots n}\right) \cdot\left(1+2^{\frac{2-n}{2}}\right) \\
& =2^{n-2} \cdot\left(1-2^{2}\right)\left(1-2^{-4}\right) \cdots\left(1-2^{1 \cdots n}\right) \cdot\left(1-2^{2 \cdot n}\right) .
\end{aligned}
$$

Par le lemme 5.7, on a :

$$
\mathscr{H}_{\mathscr{N}_{n}}=\frac{1}{2^{n \cdots 1} \cdot\left(\frac{n-2}{2}\right) !} \cdot\left|E_{\frac{r_{t}-2}{2}} \cdot B_{2} \cdots B_{n-2}\right| .
$$

i) Supposons enfin que $n \equiv 7$ mod 8 .

On finit par trouver :

$$
\begin{aligned}
\mu_{2}\left(O\left(M_{2}\right)\right) & =2^{-n \div 2} \cdot\left(1-2^{-2}\right)\left(1-2^{-4}\right) \cdots \cdots\left(1-2^{3-n}\right) \cdot\left(1+2^{\frac{1-n}{2}}\right) \\
& =\frac{2^{-n-2}}{\left(1+2^{\frac{1-n}{2}}\right)} \cdot\left(1-2^{2}\right)\left(1-2^{-4}\right) \cdots\left(1-2^{3-n}\right) \cdot\left(1-2^{1-n}\right)
\end{aligned}
$$

Le lemme 5.5 nous donme alors :

$$
\mathscr{H}_{x_{n}}=\frac{\left(2^{\frac{n}{2}}+1\right)}{2^{n} \cdot\left(\frac{n}{2}\right) !} \cdot\left|B_{2} \cdots B_{n_{2}} \quad 1\right|
$$




\section{Applications et conclusion.}

La formule de Minkowski-Sicgel est utile pour calculer le cardinal de $\mathscr{L}_{n}$ et de $\mathscr{C}_{n}$, autrement dit pour trouver le nombre de classes d'équivalences de $\mathscr{S}_{n}$.

a) Le cas de $\mathscr{C}_{n}$

Si $n=8$, on sait que $\Gamma_{8}$ défini au chapitre 1 est un élément de $\mathscr{C}_{n}$. Il est possible de voir (Bourbaki, groupe et algèbre de Lie, chap. $V I, \S 4, N^{\circ} 10$, ou alors $[4$, ch 2, p. 50] ) que

$$
\left|O\left(\Gamma_{8}\right)\right|=2^{14} \cdot 3^{5} \cdot 5^{2} \cdot 7 .
$$

Or, le théorème 5.3 nous donne:

$$
\mathscr{M C O}_{B_{8}}=\frac{1}{2^{14} \cdot 3^{5} \cdot 5^{2} \cdot 7}
$$

On en déduit donc que $\left|\mathbb{s}_{8}\right|=1$.

Si $n=16$, posons

$$
\Gamma_{16}=\left(\begin{array}{rrrrrrrrrrrrrrrr}
2 & 0 & -1 & 0 & 0 & 0 & 0 & 0 & 0 & 0 & 0 & 0 & 0 & 0 & 0 & -1 \\
0 & 2 & -1 & 0 & 0 & 0 & 0 & 0 & 0 & 0 & 0 & 0 & 0 & 0 & 0 & 0 \\
-1 & -1 & 2 & -1 & 0 & 0 & 0 & 0 & 0 & 0 & 0 & 0 & 0 & 0 & 0 & 0 \\
0 & 0 & -1 & 2 & -1 & 0 & 0 & 0 & 0 & 0 & 0 & 0 & 0 & 0 & 0 & 0 \\
0 & 0 & 0 & -1 & 2 & -1 & 0 & 0 & 0 & 0 & 0 & 0 & 0 & 0 & 0 & 0 \\
0 & 0 & 0 & 0 & -1 & 2 & -1 & 0 & 0 & 0 & 0 & 0 & 0 & 0 & 0 & 0 \\
0 & 0 & 0 & 0 & 0 & -1 & 2 & -1 & 0 & 0 & 0 & 0 & 0 & 0 & 0 & 0 \\
0 & 0 & 0 & 0 & 0 & 0 & -1 & 2 & -1 & 0 & 0 & 0 & 0 & 0 & 0 & 0 \\
0 & 0 & 0 & 0 & 0 & 0 & 0 & -1 & 2 & -1 & 0 & 0 & 0 & 0 & 0 & 0 \\
0 & 0 & 0 & 0 & 0 & 0 & 0 & 0 & -1 & 2 & -1 & 0 & 0 & 0 & 0 & 0 \\
0 & 0 & 0 & 0 & 0 & 0 & 0 & 0 & 0 & -1 & 2 & -1 & 0 & 0 & 0 & 0 \\
0 & 0 & 0 & 0 & 0 & 0 & 0 & 0 & 0 & 0 & -1 & 2 & -1 & 0 & 0 & 0 \\
0 & 0 & 0 & 0 & 0 & 0 & 0 & 0 & 0 & 0 & 0 & -1 & 2 & -1 & 0 & 0 \\
0 & 0 & 0 & 0 & 0 & 0 & 0 & 0 & 0 & 0 & 0 & 0 & -1 & 2 & -1 & 0 \\
0 & 0 & 0 & 0 & 0 & 0 & 0 & 0 & 0 & 0 & 0 & 0 & 0 & -1 & 2 & 0 \\
-1 & 0 & 0 & 0 & 0 & 0 & 0 & 0 & 0 & 0 & 0 & 0 & 0 & 0 & 0 & 4
\end{array}\right) .
$$

On peut montrer que

$$
\left|O\left(\mathrm{~L}_{8} \mathrm{H}_{\mathrm{s}}\right)\right|=2^{29} \cdot 3^{110} \cdot 5^{-1} \cdot 7^{2} \text { at }\left|O\left(\mathrm{l}_{16}\right)\right|=2^{15}
$$

Le théorème 5.3 nous permet d'allimerm que:

$$
\mathscr{M}_{16}=\frac{1}{2^{29} \cdot 3^{10} \cdot 5^{4} \cdot 7^{2}}+\frac{1}{2^{15} \cdot(16) !}
$$

donc que $\left|\mathscr{C}_{16}\right|=2$.

Si $n=24$, ça ce complique, on a que $\left|\mathscr{C}_{24}\right|=24$. La détermination de ces classes d'équivalences a été faite par H. Niemeier en 1968. La liste de ces réseaux est donnée dans [4, ch. 16 et 18]. Notons que parmi ces réseaux, l'un d'eux est particulièremont remarquable. Il s'agil du réseau de Leech. On le note souvent $\Lambda_{24}$. Il ne contient aucun vecteur tel que $\beta(x, x)=2$, en outre, l'ordre du groupe $\mathcal{O}\left(\Lambda_{24}\right)$ est

$$
2^{22} \cdot 3^{9} \cdot 5^{4} \cdot 7^{2} \cdot 11 \cdot 13 \cdot 23
$$

Le quotiont $O\left(\Lambda_{21}\right) /\{ \pm 1\}$ est le groupe Coo construit par Conway. Il s'agit d'un "groupe simple sporadique".

Pour plus de détail sur le réscau de lecech, voir $[4$, ch. 8, 12, 23, 24, 25 et 26], voir aussi $[4$, ch. 10, 11 et 12] à propos de $C o_{0}$.

Si $n=32$, Le théoreme 5.3 nous donne que $\mathscr{M}_{\mathscr{B}_{32}}>1 \cdot 10^{7}$. Puisque $|O(M)| \geq 2$ pour tout $M$, on trouve que $\mathscr{C}_{32}$ a plus de 80 millions d'éléments. In travail similaire à celui de Niemeier pour ce cas parait humainement inabordable. 
b) Le cas de $\mathscr{K}_{n}$

Si $n \leq 8$, le thórome 5.8 nous donne que

$$
\mathscr{H}_{\mathscr{W}_{n}}=\frac{1}{2^{n} \cdot n !}
$$

Munissons $Z^{n}$ de la forme définie par la matrice identité. On a alors $O\left(Z^{n}\right)=2^{n} \cdot n !$ (voir $[4$, ch. 4, §5]). On obtient done que le cardinal de $\mathscr{H}_{n}$ vaut 1 . Posons $s_{n}$, le nombre de classes d'équivalences de $\mathscr{S}_{n}$. Ce qui précède ainsi que le paragraphe $\mathrm{F}$ du premier chapitre nous donne :

$$
s_{n}=\left\{\begin{array}{lll}
1 & \text { si } n \leq 7 & \text { (ce résultat était déjà connu par Hermite) } \\
2 & \text { si } n=8 & \text { (Mordell) }
\end{array}\right.
$$

Si $n=9$, on utilise aussi cette móthode pour trouver que :

$$
\mathscr{H}_{n}=\left\{\mathrm{I}_{8} \oplus<1>, \mathrm{I}_{9}\right\}
$$

donc, $\left|\mathscr{H}_{n}\right|=s_{n}=2$.

Finalement, les connaissances actuolles sur ces cardinaux se résument à ce tableau :

\begin{tabular}{l|rrrrrrrrrrrrrrrrrrrr}
$n$ & $1 \leq n \leq 7$ & 8 & 9 & 10 & 11 & 12 & 13 & 14 & 15 & 16 & 17 & 18 & 19 & 20 & 21 & 22 & 23 & 24 & 25 & 26 \\
\hline$\left|\mathscr{C}_{n}\right|$ & 0 & 1 & 0 & 0 & 0 & 0 & 0 & 0 & 0 & 2 & 0 & 0 & 0 & 0 & 0 & 0 & 0 & 24 & 0 & 0 \\
\hline$\left|\mathscr{H}_{n}\right|$ & 1 & 1 & 2 & 2 & 2 & 3 & 3 & 4 & 5 & 6 & 9 & 13 & 16 & 28 & 40 & 68 & 117 & 273 & 665 & $?$ \\
\hline$s_{n}$ & 1 & 2 & 2 & 2 & 2 & 3 & 3 & 4 & 5 & 8 & 9 & 13 & 16 & 28 & 10 & 68 & 117 & 297 & 665 & $?$
\end{tabular}

Pour plus de détails, voir dans [4]. 


\section{APPENDICE}

\section{Deux nouvelles démonstrations de la proposition 4.5.}

Rappelons que le but est de calculer le cardinal des vecteurs de $(\pi / 8 \mathbb{Z})^{n}$ de longueur $k$ fixé. On a appelé ce nombre : $t_{k}(n)$.

\section{Lemme 1.}

Posons $\zeta=e^{\frac{2 i \pi}{8}}$ et $\Omega=\left\{\zeta^{r} \mid 0 \leq r \leq 7\right\}$. Soit $s \in \mathbb{I}$. On a :

$$
\sum_{\omega \in \Omega} \omega^{s}=\sum_{r=0}^{7}\left(\zeta^{s}\right)^{r}= \begin{cases}8 & \text { si } \zeta^{s}=1 \\ 0 & \text { sinon }\end{cases}
$$

\section{Démonstration :}

Une démonstration de ce fait est donnée dans [10, ch. 6,81 , proposition 4]. Mais bon, on peut aussi le vérifier directement.

Proposition 4.5

$$
t_{k}(n)=\left\{\begin{array}{lll}
2^{3 n-3}+2^{\frac{5 n-4}{2}} \cdot \cos \left(\frac{\pi}{4}(2 k-n)\right) & \text { si } k \neq n=(\bmod 4) \\
2^{3 n-3}+2^{\frac{3 n-1}{2}} \cdot \cos \left(\frac{\pi}{4}(2 k-n)\right)+(-1)^{(k-n) / 4} \cdot 2^{2 n-1} & \text { si } k \equiv n & (\bmod 4)
\end{array}\right.
$$

Démonstation de E. Preissmann

Posons $\Psi=\left\{(l, m) \in \mathbb{Z}^{2} \mid l, m \geq 0, l+m \leq n\right.$ et $\left.m+4 l=k\right\}$. On a :

$$
\begin{aligned}
& \left|\left\{x \in / 8 \mid x^{2}=0\right\}\right|=2 \\
& \left|\left\{x \in \cdots / 8 \cdots x^{2}=1\right\}\right|=4 \\
& \left|\left\{x \in / 8 \cdots \mid x^{2}=4\right\}\right|=2 .
\end{aligned}
$$

Un rapide raisonnement de combinatoire nous donne:

$$
\iota_{k}(n)=\sum_{l, m \in \Psi} 2^{n-l-m} 4^{m} 2^{l} \cdot\left(\begin{array}{c}
n \\
l m
\end{array}\right)=2^{n} \cdot \sum_{l, m \in \Psi} 2^{m}\left(\begin{array}{c}
n \\
l m
\end{array}\right) .
$$

Rappelons que $\left(\begin{array}{c}n \\ l m\end{array}\right)=\frac{n !}{l ! m !(n-l-m) !}$. Soient $a, b \in I$. Calculons :

$$
\begin{aligned}
\sum_{\omega \in \Omega}\left(1+\omega^{a}+2 \omega^{b}\right)^{n} \cdot \omega^{-k} & =\sum_{\omega \in \Omega} \sum_{l, m} 1^{n \cdots l \cdots m}\left(\omega^{a}\right)^{l}\left(2 \omega^{b}\right)^{m}\left(\begin{array}{c}
n \\
l m
\end{array}\right) \cdot \omega^{-k} \\
& =\sum_{l, m} \sum_{\omega \in \Omega} \omega^{a l+b m \cdots k} 2^{n}\left(\begin{array}{c}
n \\
l m
\end{array}\right) \\
& \stackrel{\operatorname{lemme} 1}{=} \sum_{\substack{l, m, \\
\text { sial+bm-k }}} 8 \cdot 2^{m}\left(\begin{array}{c}
n \\
l m
\end{array}\right)
\end{aligned}
$$

Posons $a=4$ et $b=1$. On trouve donc:

$$
\sum_{\omega, \Omega \Omega}\left(1+\omega^{1}+2 \omega\right)^{n} \cdot \omega^{k}=\sum_{l . m \in \psi} 8 \cdot 2^{m}\left(\begin{array}{c}
n \\
l m
\end{array}\right)=2^{-n-3} \cdot t_{k}(n) .
$$

Donc,

$$
t_{k}(n)=2^{n} \cdot 3 \sum_{\omega \in \Omega}\left(1+\omega^{4}+2 \omega\right)^{n} \cdot \omega^{-k}:=2^{n-3} \cdot \Xi(n, k) .
$$


Posons $\Omega_{1}=\left\{\zeta, \zeta^{3}, \zeta^{5}, \zeta^{7}\right\}$. On obtient :

$$
\Xi(n, k)=\sum_{\omega \in \Omega_{1}}(2 \omega)^{n} \omega^{-k}+(2+2 i)^{n} i^{-k}+(2-2 i)^{n}(-i)^{-k}+0+4^{n} .
$$

Or,

$$
\begin{aligned}
\sum_{\omega \in \Omega_{2} 1_{1}}(2 \omega)^{n} \omega^{-k} & =2^{n} \cdot\left(\zeta^{n-k}+\zeta^{3(n-k)}+\zeta^{5(n-k)}+\zeta^{7(n-k)}\right) \\
& =2^{n} \zeta^{n-k} \cdot\left(1+\zeta^{2(n \cdots k)}+\zeta^{4(n \cdots k)}+\zeta^{6(n-k)}\right) \\
& = \begin{cases}0 & \text { si } 4 \text { ne divise pas } n-k \\
2^{n+2} \zeta^{n-k} & \text { si } 4 \text { divise } n-k .\end{cases}
\end{aligned}
$$

La dernière égalité est un corollaire du lemme 1. Il suit que :

a) $\Xi(n, k)=2^{2 n}+2^{n+1} \cdot \Re\left((1+i)^{n} i^{-k}\right)$ si 4 ne divise pas $n-k$.

b) $\Xi(n, k)=2^{2 n}+2^{n-1} \cdot \Re\left((1+i)^{n} i^{-k}\right)+(-1)^{(k-n) / 4} \cdot 2^{n+2}$ si 4 divise $n-k$.

Sachant que $1+i=\sqrt{2} \cdot \zeta$, et que $\Re\left(\zeta^{n-2 k}\right)=\cos \left(\frac{\pi}{4}(2 k-n)\right)$, on conclut.

\section{Démonstation de H. Joris}

Posons $e_{8}(z)=\exp \left(\frac{2 \pi \mathbf{i} z}{8}\right)$. Nous avons $\mathrm{i}=e_{8}(2)$ et $1+\mathrm{i}=\sqrt{2} e_{8}(1)$, et

$$
\sum_{s=0}^{7} \mathrm{es}_{8}\left(s\left(\sum_{k=1}^{n} m_{k}^{2}-r\right)\right)= \begin{cases}8 & \text { si } \sum_{k=1}^{n} m_{k}^{2} \equiv r \quad(\bmod 8) \\ 0 & \text { sinon }\end{cases}
$$

Nous en déduisons

$$
\begin{aligned}
8 t_{r}(n) & =\sum_{m_{1}=0}^{7} \sum_{m_{2}=0}^{7} \ldots \sum_{m_{n}=0}^{7} \sum_{s=0}^{7} e_{8}\left(s\left(\sum_{k=1}^{n} m_{k}^{2}-r\right)\right) \\
& =\sum_{s=0}^{7} e_{8}(-r s) \sum_{m_{1}}^{7} \sum_{0}^{7} \ldots \sum_{m_{2}=0}^{7} e_{8}\left(s m_{1}^{2}\right) e_{8}\left(s m_{2}^{2}\right) \ldots e_{8}\left(s m_{n}^{2}\right)=\sum_{s=0}^{7} e_{8}(-r s)(\Omega(s))^{n},
\end{aligned}
$$

où

$$
\Omega(s)=\sum_{m=0}^{7} \mathrm{e}_{8}\left(s m^{2}\right)=2+2(-1)^{s}+4 \mathrm{e}_{8}(s)= \begin{cases}4 \mathrm{e}_{8}(s) & \text { si } s=2 t+1 \text { est impair } \\ 4\left(1+\mathrm{i}^{t}\right) & \text { si } s=2 t \text { est pair. }\end{cases}
$$

Il suit

$$
\begin{aligned}
8 t_{r}(n) & =\sum_{t=0}^{3} e_{8}(-r(2 t+1)) \Omega(2 t+1)^{n}+\sum_{i=0}^{3} e_{8}(-r(2 t)) \Omega(2 t)^{n} \\
& =\sum_{t=0}^{3} e_{8}(-r(2 t+1)) 4^{n} e_{8}((2 t+1) n)+\sum_{i=0}^{3} \mathrm{i}^{\cdots r t} 4^{n}\left(1+\mathrm{i}^{l}\right)^{n} \\
& =4^{n}\left(\cos _{8}(n-r) \sum_{t=0}^{3} \mathrm{i}^{l(n)}+\sum_{t=0}^{3} \mathrm{i}^{-r t} 4^{n}\left(1+\mathrm{i}^{l}\right)^{n}\right) \\
& =4^{n}\left(\cos (n-r) \sum_{t=0}^{3} \mathrm{i}^{l(n \cdot r)}+2^{n}+(1+\mathrm{i})^{n} \mathrm{i}^{-r}+(1-\mathrm{i})^{n} \mathrm{i}^{r}\right) \\
& \left.=4^{n}\left(\operatorname{es}_{8}(n-r) \sum_{t=0}^{3} \mathrm{i}^{l(n-r)}+2^{n}+2 \Re\left(\sqrt{2}^{n} \mathrm{e}_{8}(n-2 r)\right)\right)\right) \\
& =4^{n}\left(e_{8}(n-r) \sum_{t=0}^{3} \mathrm{i}^{l(n-r)}+2^{n}+2 \sqrt{2}{ }^{n} \cos \left(\frac{n-2 r}{4} \pi\right)\right) \\
& = \begin{cases}4^{n}\left(4(-1)^{(n-r) / 4}+2^{n}+2^{i+n / 2} \cos \left(\frac{n \pi}{4}-\frac{r \pi}{2}\right)\right) & \text { si } n \equiv r \bmod 4 ; \\
4^{n}\left(2^{n}+2^{l i n / 2} \cos \left(\frac{n \pi}{4}-\frac{r \pi}{2}\right)\right) & \text { sinon. }\end{cases}
\end{aligned}
$$


Finalement nous obtenors

$$
t_{r}(n)= \begin{cases}2^{3 n-3}+2^{2 n-2+n / 2} \cos \left(\frac{n \pi}{4}-\frac{r \pi}{2}\right)+2^{2 n-1}(-1)^{(n-r) / 4} & \text { si } n \equiv r \bmod 4 ; \\ 2^{3 n-3}+2^{2 n-2+n / 2} \cos \left(\frac{n \pi}{4}-\frac{r \pi}{2}\right) & \text { sinon } .\end{cases}
$$




\section{Bibliographie}

[1] ABRAMOWITZ, M. \& STEGUN, I.A. : Handbook of Mathematical Functions. National Bureau of Standard Appl. Math. Series 55, U.S. Dept. of Commerce. Washington. 1970

[2] BAEZA, R. : Quadralic Forms over semilocal rings. Lecture Notes in Mathematics Vol 655. SpringerVerlag. Berlin, Heidelberg, New York. 1978.

[3] CASSELS, J.W.S. : Rational Quadratic Forms. London Math. Society. 1978.

[4] CONWAY, J.H. \& SLOANE, I.J.A. : Sphere Packing. Springer-Verlag. Berlin, IJeidelberg, New York. 1988 .

[5] EICHLER, M. : Quadralische Formen und orthogonale Gruppen. Grundlehren des Math. Wiss. zu Berlin. No. 63. Springer-Verlag. Berlin, Heidelberg, New York. 1952.

[6] DIEUDONNE, J. : La Géométrie des groupes classiques. Springer-Verlag. Berlin, Göttingen, Heidelberg. 1955.

[7] KNESER, M. : Quadratische lormen. Vorlesung SS/WS 1973-74, Mathematisches Institut. Göttingen. 1974.

[8] MILNOR, J. \&. HUSEMOLLER, D. : Symmelric bilinear forms. Ergebnisse der Math. No. 73. SpringerVerlag. Berlin, Heidelberg, New York. 1973.

[9] PALL, G. : The Weight of a Cicnus of Positive n-ary Quadralic forms. Proce of sympos, in pure Math. No. 8 pp. 95 10\%. 1965

[10] SERRE, J.-P. : Cours d'arithmétique. Collection SUP No.2. Presses Universitaires de France. Paris. 1970.

[11] SIEGEL, C.L. : Über die Analyizsche Theorie der Quadratische Formen. Annals of Mathematics No. 36. 1935.

[12] WATSON, G.L. : The 名-adic density of a quadratic form. Mathematika No. 23 pp. 94-106. 1976. 